Japan. J. Math.

Vol. 3, No. 1. 1977

\title{
Invariant eigendistributions of Laplace operators on real simple Lie groups, IV
}

\author{
Explicit forms of the characters of discrete series \\ representations for $S p(n, R)$
}

\author{
By Takeshi HIRAI
}

(Received August 4, 1975)

\section{Introduction}

In the previous papers [4(c), (d)], we studied the invariant eigendistributions on real semisimple Lie groups from a general stand point. Let $G$ be such a group and $G^{\prime}$ its subset of all regular elements. In [4(c)], we obtained a necessary and sufficient condition that an invariant analytic function on the open dense subset $G^{\prime}$ defines canonically an invariant eigendistribution on $G$. In [4(d)], we gave a method $\boldsymbol{T}$ of constructing a complete system of such distributions and also a method $T^{\prime}$ of constructing a complete system of tempered such distributions. Now in the present paper, we give the explicit forms of the characters of the irreducible unitary representations in the discrete series. Here we are mainly concerned with the groups $S p(n, \boldsymbol{R})$. This gives us very important examples of invariant eigendistributions.

We know by the results of Harish-Chandra [2(b)] that $G$ has the discrete series representations if and only if it has a compact Cartan subgroup. Moreover the characters of such representations are characterized as the tempered invariant eigendistributions on $G$ having certain special forms on the compact Cartan subgroup. For other Cartan subgroups not conjugate to the compact one, the explicit forms of these characters on them have been left undetermined in general except for $S U(p, q)$ in [4(a)] or for some lower rank groups. When we are restricted ourselves to the classical type, the simple groups having compact Cartan subgroups are up to local isomorphisms, $S p(n, \boldsymbol{R}), \operatorname{Spin}(p, q)$ ( $p$ or $q$ even), $S O^{*}(2 n), S p(p, q)$ and $S U(p, q)$ in the notation in [3]. For any one of $S O^{*}(2 n)$ and $S p(p, q)$, its Cartan subgroups are all connected, and the order $<$ in the set Car $(G)$ of all conjugate classes under $G$ of Cartan subgroups is linear (for the definition of the order, see $\S 1)$. So the situation is quite similar to that for $S U(p, q)$ and can 
be treated just as in [4(a)]. The groups $\operatorname{Spin}(p, q)$ will be studied in the succeeding paper [4(e)].

The method employed here was explained briefly in [4(b)], and the explicit forms of the characters of discrete series representations of $S p(2, R)$ were also given there. Such studies on the groups $S p(n, \boldsymbol{R})$ were also recently made by W. Schmid in [5(a), (b)], but the method employed there is quite different from ours and, if we limit ourselves to the present problem only, the results obtained there is not sufficiently complete as he himself remarked it [5(b)].

The contents of the present paper are as follows. In $\S 1$ we give, as Theorem 1, a version of the necessary and sufficient condition in [4(c)] mentioned above. This plays a fundamental role in our method. Let us explain this. The method $T^{\prime}$ in [4(d)] of constructing tempered invariant eigendistributions contains certain inductive processes according to the order $<$ in $\operatorname{Car}(G)$ (for the details, see $[4(d), \S 8]$ ). These inductive processes can be directly achieved when the structure of Car $(G)$ is fairly simple, and so the method $T^{\prime}$ gives us the explicit forms of the characters when the rank of $G$ is small. But this direct calculation is impracticable for $S p(n, \boldsymbol{R})$ when $n$ is large. Hence we proceed as follows. Carefully taking into account the explicitly calculated results for $n=2,3$ and 4 , we presume general formulas for the explicit forms of the characters. Then we test by Theorem 1 whether the presumed formulas give actually tempered invariant eigendistributions or not. This is exactly what we have done in this paper.

In $\S \S 2-3$, we study the structures of $S p(n, R)$ necessary to apply Theorem 1 for these groups. In $\S 4$ we determine explicitly certain differential equations appearing in Theorem 1 , and then in $\S 5$ write down Theorem 1 by means of explicit parameters and variables for $S p(n, \boldsymbol{R})$ (Theorem 4). In $\S 6$ the explicit forms of the characters are given without proofs for $S p(2, R)$ and $S p(3, R)$. These results for $n=2,3$ help us to understand well the general case where the situation is very complicated. In $\S 7$ we give unified explicit formulas of the characters for $S p(n, \boldsymbol{R})$ (Theorem 5). These formulas are rather simple and give us the best expressions from a certain point of view (see $\S 7.2$ ). In $\S 8$, a proof of these formulas are given. In the last section, $\S 9$, some special subfamilies of the discrete series representations containing the holomorphic ones are studied briefly.

\section{CONTENTS}

$\S 1$. Method of computation .......................... 3

$\S 2$. Structures of Cartan subgroups and Weyl groups .......... 9

$\S 3 . \quad$ Orders in $\operatorname{Car}(G)$ and $\operatorname{Car}^{0}(G) \ldots \ldots \ldots \ldots \ldots \ldots \ldots \ldots \ldots \ldots$ 
4. Differential equations connecting $\tilde{\kappa}^{\natural}$ and $\tilde{\kappa}^{\mathfrak{h}_{\alpha}} \ldots \ldots \ldots \ldots \ldots 17$

$\S 5$. The equations on the system of functions $\tilde{\kappa}_{j}^{k, l}, \mathrm{~s} \ldots \ldots \ldots \ldots 25$

$\S 6$. Explicit formulas for $S p(2, \boldsymbol{R})$ and $S p(3, \boldsymbol{R}) \ldots \ldots \ldots \ldots \ldots 28$

$\S 7$. Explicit formulas for $S p(n, R) \ldots \ldots \ldots \ldots \ldots \ldots \ldots \ldots \ldots \ldots$

$\S 8$. Proof of the main theorem.................... 34

$\S 9$. Some simple cases $\ldots \ldots \ldots \ldots \ldots \ldots \ldots \ldots \ldots \ldots \ldots \ldots \ldots$

References ............................ 47

\section{§1. Method of computation}

1.1. Let $G$ be a connected real semisimple Lie group. We know that an invariant eigendistribution $\pi$ on $G$ essentially coincides with an analytic function $\pi^{\prime}$ on the dense open subset $G^{\prime}$ of all regular elements of $G$ [2(b), Th. 2]. Clearly $\pi^{\prime}$ is invariant under the inner automorphisms of $G$. Conversely, assume that we are given an invariant analytic function $\pi^{\prime}$ on $G^{\prime}$. What is the condition that $\pi^{\prime}$ defines canonically an invariant eigendistribution on $G$ ? In the previous paper [4(c)], we gave a necessary and sufficient condition for it. Our method of computation employed here is based upon this fundamental theorem [4(c), §4, Th. 3]. Let us explain this more in detail.

1.2. We introduce some notations. Let $g$ be the Lie algebra of $G, \mathfrak{h}$ a Cartan subalgebra of $g$, and $H^{\natural}$ the Cartan subgroup of $G$ corresponding to $\mathfrak{h}$. Introduce a lexicographic order in the set of roots $\left(\mathfrak{g}_{c}, \mathfrak{h}_{c}\right)$ (or simply of $\mathfrak{h}$ ), and denote by $P(\mathfrak{h})$ (or $S_{R}(\mathfrak{h})$ resp.) the set of all positive roots (or of positive real roots resp.). Here $\mathfrak{g}_{c}$ and $\mathfrak{h}_{c}$ denote the complexifications of $\mathfrak{g}$ and $\mathfrak{h}$ respectively, and a root $\alpha$ of $\left(\mathfrak{g}_{c}, \mathfrak{h}_{c}\right)$ is called real if it takes only real values on $\mathfrak{h}$.

We may assume, taking an appropriate finite covering of $G$ if necessary, that $G$ is acceptable in the sense of Harish-Chandra [2(a), p. 483]. This means that there exists a connected Lie group $G_{c}$ with Lie algebra $g_{c}$ such that the natural injection of $g$ into $g_{c}$ can be lifted up to a homomorphism $j$ of $G$ into $G_{c}$, and that there exists a homomorphism $\xi_{\rho}$ of the Cartan subgroup $\exp \mathfrak{h}_{c}$ of $G_{c}$ into $C^{*}=C-\{0\}$ whose differential is equal to $\rho=2^{-1} \sum_{\alpha>0} \alpha$. Denote again by $\xi_{\rho}$ the homomorphism $\xi_{\rho} \circ j$ of $H^{\natural}$ into $C^{*}$, and define a homomorphism $\xi_{\alpha}$ for any root $\alpha$ of $\mathfrak{h}$ by

$$
\operatorname{Ad}(h) X_{\alpha}=\xi_{\alpha}(h) X_{\alpha} \quad\left(h \in H^{\mathfrak{y}}\right),
$$

where $X_{\alpha} \in \mathfrak{g}_{c}$ is a non-zero root vector for $\alpha$. Put for $h \in H^{\mathfrak{h}}$, 


$$
\begin{aligned}
& \Delta^{\mathfrak{G}}(h)=\xi_{\rho}(h) \prod_{\alpha \in P(\mathfrak{G})}\left(1-\xi_{\alpha}(h)^{-1}\right), \\
& \Delta_{R}^{\prime}(h)=\prod_{\alpha \in S_{R(\mathfrak{G})}}\left(1-\xi_{\alpha}(h)^{-1}\right) .
\end{aligned}
$$

For any subset $F$ of $G$ or $g$, denote by $W_{G}(F)$ the group of transformations on $F$ induced by the inner automorphisms of $G$ or $g$ respectively leaving $F$ invariant. Then $W_{G}(\mathfrak{h})$ can be considered canonically as a subgroup. of the Weyl group $W\left(\mathfrak{h}_{c}\right)$ of $\mathfrak{g}_{c}$ acting on $\mathfrak{h}_{c}$. There exists a natural homomorphism of $W_{G}\left(H^{\mathfrak{\xi}}\right)$ onto $W_{G}(\mathfrak{h})$, denoted as $W_{G}\left(H^{\mathfrak{\natural}}\right) \ni \omega \rightarrow \bar{\omega}=w \in W_{G}(\mathfrak{h})$. Put $\tilde{\varepsilon}(\omega)=\operatorname{sgn}(\bar{\omega})$, the sign of $\bar{\omega}$ as an element of $W\left(\mathfrak{h}_{c}\right)$, then

$$
\Delta^{\mathfrak{h}}(\omega h)=\tilde{\varepsilon}(\omega) \Delta^{\mathfrak{G}}(h) \quad\left(\omega \in W_{G}\left(H^{\mathfrak{\natural}}\right), h \in H^{\mathfrak{l}}\right) .
$$

Let $\pi^{\prime}$ be an invariant analytic function on $G^{\prime}$. We put

$$
\tilde{\kappa}^{\mathfrak{h}}(h)=\Delta^{\mathfrak{h}}(h) \pi^{\prime}(h) \quad\left(h \in H^{\prime \mathfrak{h}}=H^{\mathfrak{h}} \cap G^{\prime}\right) .
$$

Then it follows from (1.2) that

$$
\tilde{\kappa}^{\mathfrak{\natural}}(\omega h)=\tilde{\varepsilon}(\omega) \tilde{\kappa}^{\natural}(h) \quad\left(h \in H^{\prime \mathfrak{\natural}}, \omega \in W_{G}\left(H^{\mathfrak{\natural}}\right)\right) .
$$

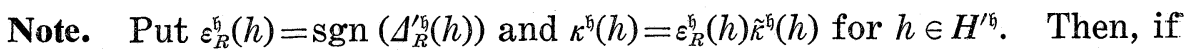
$\pi^{\prime}$ comes canonically from an invariant eigendistribution $\pi$ on $G$, every function $\kappa^{\mathfrak{h}}$ can be extended to a continuous function on the whole $H^{\mathfrak{b}}$, but $\tilde{\kappa}^{\mathfrak{b}}$ cannot in general. In this paper we do not use the functions of the form $\kappa^{\text {b }}$.

Let $I\left(\mathfrak{h}_{c}\right)$ be the subalgebra of the symmetric algebra $S\left(\mathfrak{h}_{c}\right)$ of $\mathfrak{h}_{c}$ consisting of all elements invariant under $W\left(\mathfrak{h}_{c}\right)$. A linear form $\mu$ of $\mathfrak{h}_{c}$ determines. cánonically a homomorphism $\lambda_{\mu}$ of $I\left(\mathfrak{h}_{c}\right)$ into $C$. Moreover any homomorphism $\lambda$ of $I\left(\mathfrak{h}_{c}\right)$ into $C$ is expressed as $\lambda=\lambda_{\mu}$ for some $\mu$. We know that $\lambda_{\mu}$. $=\lambda_{\mu^{\prime}}$ if and only if $\mu^{\prime}=w \mu$ for some $w \in W\left(\mathfrak{h}_{c}\right)$. Let $\mathfrak{h}^{1}$ be another Cartan. subalgebra of $\mathrm{g}$. A homomorphism $\lambda_{\mu 1}$ of $I\left(\mathfrak{h}_{c}^{1}\right)$ is said to be consistent with such a one $\lambda_{\mu}$ of $I\left(\mathfrak{h}_{c}\right)$ if there exists an inner automorphism $\nu$ of $\mathfrak{g}_{c}$ such that $\nu \mathfrak{h}_{c}=\mathfrak{h}_{c}^{1}$ and $\nu \mu=\mu^{1}$, where $\nu \mu=\mu \circ \nu^{-1}$.

Let $\operatorname{Car}(\mathfrak{g})$ and $\operatorname{Car}(G)$ be the sets of conjugate classes under $G$ of Cartan subalgebras of $g$ and Cartan subgroups of $G$ respectively. Let $\left\{\mathfrak{h}^{1}, \mathfrak{h}^{2}, \cdots, \mathfrak{h}^{q}\right\}$ be a complete system of representatives of the conjugate classes in Car (g). Then $\left\{H^{1}, H^{2}, \cdots, H^{q}\right\}$ is such a one for $\operatorname{Car}(G)$, where $H^{r}$ $=H^{\natural^{r}}$. Put $\tilde{\kappa}^{r}=\tilde{\kappa}^{\tilde{k}^{r}}$, then the system of functions $\tilde{\kappa}^{1}, \tilde{\kappa}^{2}, \cdots, \tilde{\kappa}^{q}$ determines the invariant function $\pi^{\prime}$ on $G^{\prime}$ completely.

Assume that $\mathfrak{h}$ is conjugate to $\mathfrak{h}^{r}$ under $G$. Take an element $g \in G$ such that $\operatorname{Ad}(g) \mathfrak{h}=\mathfrak{h}^{r}$, and put

$$
P(\mathfrak{h})^{\nu}=\{\nu \alpha ; \alpha \in P(\mathfrak{h})\},
$$


where $\nu=\operatorname{Ad}(g) \mid \mathfrak{h}$. Then there exists a unique $w \in W\left(\mathfrak{h}_{c}^{r}\right)$ such that $P\left(\mathfrak{h}^{r}\right)^{w}$ $=P(\mathfrak{h})^{\nu}$. It follows from (1.2) and the invariance of $\pi^{\prime}$ that

$$
\tilde{\kappa}^{\mathfrak{g}}(h)=\operatorname{sgn}(w) \tilde{\kappa}^{r}\left(h^{g}\right) \quad\left(h \in H^{\prime \mathfrak{l}}\right),
$$

where $h^{g}=g h g^{-1} \in H^{\prime r}=H^{\prime \xi^{r}}$.

Take a real root $\alpha$ of $\mathfrak{h}$, and let $H_{\alpha}$ be the element of $\mathfrak{h}$ corresponding to $\alpha$ under the Killing form of $g_{c}$. Then we can choose root vectors $X_{\alpha}$ and $X_{-\alpha}$ from $g$ in such a way that $\left[X_{\alpha}, X_{-\alpha}\right]=H_{\alpha}$. Put

$$
\begin{gathered}
H_{\alpha}^{\prime}=2|\alpha|^{-2} H_{\alpha}, \quad X_{ \pm \alpha}^{\prime}=\sqrt{2}|\alpha|^{-1} X_{ \pm \alpha}, \\
\nu_{\alpha}=\exp \left\{-\sqrt{-1} \frac{\pi}{4} \operatorname{ad}\left(X_{\alpha}^{\prime}+X_{-\alpha}^{\prime}\right)\right\},
\end{gathered}
$$

where $|\alpha|$ denotes the length of $\alpha$ with respect to the Killing form. Then the Cartan subalgebra

$$
\mathfrak{h}_{\alpha}=\nu_{\alpha}\left(\mathfrak{h}_{c}\right) \cap \mathrm{g}
$$

of $\mathfrak{g}$ is not conjugate to $\mathfrak{h}$ under $G$. The $\operatorname{root} \beta=\nu_{\alpha} \alpha$ of $\mathfrak{h}_{\alpha}$ is singular imaginary (for the definition, see [2(a), p. 555]), and

$$
H_{\beta}^{\prime}=2|\beta|^{-2} H_{\beta}=\sqrt{-1}\left(X_{\alpha}^{\prime}-X_{-\alpha}^{\prime}\right) .
$$

Let $\sigma_{\alpha}$ be the hyperplane of $\mathfrak{h}$ defined by $\alpha(X)=0$, then

$$
\mathfrak{h}=\sigma_{\alpha}+R H_{\alpha}^{\prime}, \quad \mathfrak{h}_{\alpha}=\sigma_{\alpha}+R \sqrt{-1} H_{\beta}^{\prime} .
$$

Moreover put for any root $\gamma$ of $\mathfrak{h}$,

$$
\begin{aligned}
& \Sigma_{\gamma}=\left\{h \in H^{\mathfrak{G}} ; \xi_{\gamma}(h)=1\right\}, \\
& \Sigma_{\gamma}^{\prime}=\left\{h \in \Sigma_{\gamma} ; \xi_{\delta}(h) \neq 1 \quad \text { for any } \operatorname{root} \delta \neq \pm \gamma\right\} .
\end{aligned}
$$

Then $H^{\mathfrak{G}} \cap H^{\mathfrak{h}_{\alpha}}=\Sigma_{\alpha}=\Sigma_{\beta}$, where $\Sigma_{\beta} \subset H^{\mathfrak{h}_{\alpha}}$ is defined similarly.

1.3. Now let us state the fundamental theorem in the form convenient to our later application.

THEOREM A. An invariant analytic function $\pi^{\prime}$ on $G^{\prime}$ defines canonically an invariant eigendistribution on $G$ if and only if it satisfies the following three conditions.

(a) For every $r(1 \leqslant r \leqslant q)$, the function $\tilde{\kappa}^{r}$ on $H^{\prime r}$ corresponding to $\pi^{\prime}$ can be extended to an analytic function on $H^{\prime r}(R)=\left\{h \in H^{r} ; \Delta_{R}^{\prime \xi^{r}}(h) \neq 0\right\}$.

(b) Every $\tilde{\kappa}^{r}$ satisfies the equations

$$
D \tilde{\kappa}^{r}=\lambda^{r}(D) \tilde{\kappa}^{r} \quad\left(D \in I\left(\mathfrak{G}_{c}^{r}\right)\right),
$$


where $D$ is considered canonically as a left invariant differential operator on $H^{r}$, and $\lambda^{r}$ 's are homomorphisms of $I\left(\mathfrak{h}_{c}^{r}\right)^{\text {'s }}$ into $C$ mutually consistent for $1 \leqslant r \leqslant q$.

(c) For any $r$, put $\mathfrak{h}=\mathfrak{h}^{r}$ and let $\alpha \in S_{R}(\mathfrak{h})$. Assume that $P\left(\mathfrak{h}_{\alpha}\right)=P(\mathfrak{h})^{\nu \alpha}$, then for any integer $M \geqslant 0$,

$$
\left(H_{\alpha}^{\prime}\right)^{2 M+1} \tilde{\kappa}^{\mathfrak{G}}(h)=\left(H_{\beta}^{\prime}\right)^{2 M+1} \tilde{\kappa}^{\mathfrak{G} \alpha}(h) \quad\left(h \in \Sigma_{\alpha}^{\prime}\right),
$$

where the two sides denote the limit values at $h$ which exist under the conditions (a) and (b). Moreover it is sufficient to take only $M=0$.

1.4. Similarly as the method $\boldsymbol{T}$ of constructing invariant eigendistributions, given in $[4(d), \S 3]$, we give the method of computation in the following way.

Let $\operatorname{Car}^{0}(G)$ be the set of conjugate classes under $G$ of the connected components of Cartan subgroups of $G$. Choose a complete system of representatives $\left\{H_{j}^{r} ; 1 \leqslant j \leqslant n_{r}, 1 \leqslant r \leqslant q\right\}$ of the classes in $\operatorname{Car}^{0}(G)$ in such a way that $H_{j}^{r} \subset H^{r}$ for $1 \leqslant j \leqslant n_{r}$. For any $H_{j}^{r}$, let $F_{+}$be a connected component of $H_{j}^{r} \cap H^{\prime r}(R)$ given as

$$
F_{+}=\left\{h \in H_{j}^{r} ; 1-\xi_{\alpha}(h)^{-1}>0 \text { for any } \alpha \in S_{R}\left(\mathfrak{h}^{r}\right)\right\} .
$$

Put $\tilde{\kappa}_{j}^{r}=\tilde{\kappa}^{r} \mid F_{+}$, where $F_{+} \subset H_{j}^{r}$. Then $\tilde{\kappa}^{r}$ is completely determined by $\left\{\tilde{\kappa}_{j}^{r} ; 1 \leqslant j \leqslant n_{r}\right\}$. In fact, put for any real root $\alpha$,

$$
\omega_{\alpha} h=g_{\alpha} h g_{\alpha}^{-1} \quad\left(h \in H^{r}\right),
$$

where $g_{\alpha}=\exp \frac{\pi}{2}\left(X_{\alpha}^{\prime}-X_{-\alpha}^{\prime}\right) \in G$. Then $\omega_{\alpha} \in W_{G}\left(H^{r}\right)$. Moreover let $\Pi\left(F_{+}\right)$be: the set of $\alpha \in S_{R}\left(\mathfrak{G}^{r}\right)$ which give the boundary components of $F_{+}$of the highest dimension by $\xi_{\alpha}(h)=1$. Then the group generated by $\left\{\omega_{\alpha} ; \alpha \in \Pi\left(F_{+}\right)\right\}$ transforms $F_{+}$on any connected component of $H_{j}^{r} \cap H^{\prime r}(R)$ (see [4(d), § 3]).

Every $\tilde{\kappa}_{j}^{r}$ must satisfy

$$
\tilde{\kappa}_{j}^{r}(\omega h)=\tilde{\varepsilon}(\omega) \tilde{\kappa}_{j}^{r}(h) \quad\left(h \in F_{+}, \omega \in W_{G}\left(F_{+}\right)\right) .
$$

Conversely, as is seen in [4(d), Lem. 3.2], if a system of analytic functions. $\tilde{\kappa}_{j}^{r}$ on $F_{+} \subset H_{j}^{r}$ for $1 \leqslant j \leqslant n_{r}$ satisfies (1.15), then we get an analytic function. $\tilde{\kappa}^{r}$ on $H^{\prime r}(R)$ which extends $\tilde{\kappa}_{j}^{r}$ 's and satisfies

$$
\tilde{\kappa}^{r}(\omega h)=\tilde{\varepsilon}(\omega) \tilde{\kappa}^{r}(h) \quad\left(h \in H^{\prime r}(R), \omega \in W_{G}\left(H^{r}\right)\right) .
$$

To rewrite the condition (c) in Theorem A by means of $\tilde{\kappa}_{j}^{r}$ 's, we introduce certain orders in $\operatorname{Car}(G)$ and $\operatorname{Car}^{0}(G)(\mathrm{cf} .[4(\mathrm{c}), \S 3])$. Let $\mathfrak{h}$ be a Cartan 
subalgebra of $\mathfrak{g}$ and $\alpha$ a real root of $\mathfrak{h}$. Then we write $\mathfrak{h} \stackrel{\alpha}{\longrightarrow} \mathfrak{h}_{\alpha}$ and $[\mathfrak{h}] \stackrel{\alpha}{\longrightarrow}$ $\left[\mathfrak{G}_{\alpha}\right]$, where $[\mathfrak{h}]$ denotes the conjugate class of $\mathfrak{h}$ in Car $(\mathfrak{g})$. Introduce in Car $(\mathfrak{g})$ an order $<$ by defining $[\mathfrak{h}]<\left[\mathfrak{h}_{\alpha}\right]$ and extending it transitively. Transferring this order onto $\operatorname{Car}(G)$, we get an order $<$ in it. We know that there exist uniquely a maximal element and a minimal one in $\operatorname{Car}(G)$, [4(c), §3].

Let $H_{n}^{\natural}$ and $H_{m}^{\varphi_{\alpha}}$ be connected components of $H^{\natural}$ and $H^{\natural_{\alpha}}$ respectively.

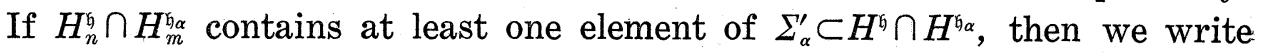
$H_{n}^{\natural} \stackrel{\alpha}{\longrightarrow} H_{m}^{\xi_{\alpha}}$ and $\left[H_{n}^{\natural}\right] \stackrel{\alpha}{\longrightarrow}\left[H_{m}^{\xi_{\alpha}}\right]$, where $\left[H_{n}^{\natural}\right]$ denotes the conjugate class of $H_{n}^{\xi^{\prime}}$ in $\operatorname{Car}^{0}(G)$. Introduce in $\operatorname{Car}^{0}(G)$ an order $\prec$ by defining $\left[H_{n}^{\mathfrak{\xi}}\right] \prec\left[H_{m}^{\mathfrak{b}_{\alpha}}\right]$ and extending it transitively.

In the following, we employ the following notation. Let $\mathfrak{h}^{\prime}$ be another Cartan subalgebra and $H_{p}^{\xi^{\prime}}$ a connected component of $H^{\mathfrak{G}^{\prime}}$. If $\left[H_{n}^{\mathfrak{G}}\right] \prec\left[H_{p}^{\mathfrak{G}^{\prime}}\right]$ and $H_{n}^{\xi} \stackrel{\alpha}{\longrightarrow} g^{-1} H_{p}^{\xi^{\prime}} g$ for $g \in G$, then we express this situation by

$$
H_{n}^{\mathfrak{\xi}} \stackrel{\alpha, g}{\longrightarrow} H_{p}^{\mathfrak{G}^{\prime}}
$$

In this case, $\mathfrak{h}^{\prime}=\operatorname{Ad}(g) \mathfrak{h}_{\alpha}$ by definition, and $\Sigma_{\alpha} \cap H_{n}^{\mathfrak{G}} \subset \Sigma_{\beta} \cap g^{-1} H_{p}^{\mathfrak{y}^{\prime}} g$, where $\beta=$ $\nu_{\alpha} \alpha$, the singular imaginary root of $\mathfrak{h}_{\alpha}$. We see that for any $H_{j}^{r}$ and $\alpha \in \Pi\left(F_{+}\right)$. with $F_{+} \subset H_{j}^{r}$, there exists exactly one $H_{k}^{s}$ such that $H_{j}^{r} \stackrel{\alpha, g}{\longrightarrow} H_{k}^{s}$ for some $g \in G$ (cf. [4(c), §3]). In this case put $\mathfrak{h}=\mathfrak{h}^{r}$, then $\mathfrak{h}^{s}=\operatorname{Ad}(g) \mathfrak{h}_{\alpha}$ and there exists a unique $w \in W\left(\mathfrak{G}_{c}^{s}\right)$ such that

$$
P\left(\mathfrak{h}_{\alpha}\right)^{\nu}=P\left(\mathfrak{h}^{s}\right)^{w}
$$

where $P\left(\mathfrak{h}_{\alpha}\right)=P(\mathfrak{h})^{\nu \alpha}$ and $\nu=\operatorname{Ad}(g) \mid \mathfrak{h}_{\alpha}$. Hence $\tilde{\kappa}^{\mathfrak{h}_{\alpha}}$ for $P\left(\mathfrak{h}_{\alpha}\right)$ is expressed by (1.5) as

$$
\tilde{\kappa}^{\mathfrak{h}_{\alpha}}(h)=\operatorname{sgn}(w) \tilde{\kappa}^{s}\left(h^{g}\right) \quad\left(h \in H^{\prime h_{\alpha} \alpha}\right),
$$

where $h^{g}=g h g^{-1}$. Put $\tilde{\beta}=\nu \beta$, then this is a singular imaginary root of $\mathfrak{h}^{s}$ and $H_{\tilde{\beta}}^{\prime}=\nu H_{\beta}^{\prime}=\operatorname{Ad}(g) H_{\beta}^{\prime}$.

Thus we get the following version of Theorem $A$ which will be employed as a method of computation.

THEOREM 1. Let $\left\{H_{j}^{r} ; 1 \leqslant j \leqslant n_{r}, 1 \leqslant r \leqslant q\right\}$ be a complete system of representatives of the elements in $\operatorname{Car}^{0}(G)$ given above. Assume that a system. of functions $\left\{\tilde{\kappa}_{j}^{r} ; 1 \leqslant j \leqslant n_{r}, 1 \leqslant r \leqslant q\right\}$ is given, where $\tilde{\kappa}_{j}^{r}$ is an analytic function on $\mathrm{F}_{+} \subset \mathrm{H}_{j}^{r}$. If it satisfies the following three conditions, it determines canonically an invariant eigendistribution on $G$.

(i) For every $r$ and $j$, 


$$
\tilde{\kappa}_{j}^{r}(\omega h)=\tilde{\varepsilon}(\omega) \tilde{\kappa}_{j}^{r}(h) \quad\left(h \in F_{+}, \omega \in W_{G}\left(F_{+}\right)\right) .
$$

(ii) For every $r$ and $j$,

$$
D \tilde{\kappa}_{j}^{r}(h)=\lambda^{r}(D) \tilde{\kappa}_{j}^{r}(h) \quad\left(h \in F_{+}, D \in I\left(\mathfrak{h}_{c}^{r}\right)\right),
$$

where $\lambda^{r}$ 's are as in Theorem A.

(iii) For any $H_{j}^{r}$ and $\alpha \in \Pi\left(F_{+}\right)$with $F_{+} \subset H_{j}^{r}$, there exist a unique $H_{p}^{s}$ such that $H_{j}^{r} \stackrel{\alpha, g}{\longrightarrow} H_{p}^{s}$ for some $g \in G$, and a unique $w \in W\left(\mathfrak{G}_{c}^{s}\right)$ such that (1.18) holds. Then for any $h \in \Sigma_{\alpha}^{\prime} \cap \mathrm{Cl}\left(F_{+}\right)$and $M=0,1,2, \cdots$,

$$
\left(H_{\alpha}^{\prime}\right)^{2 M+1} \tilde{\kappa}_{j}^{r}(h)=\operatorname{sgn}(w)\left(H_{\tilde{\beta}}^{\prime}\right)^{2 M+1} \tilde{\kappa}_{p}^{s}\left(h^{g}\right),
$$

where $\mathrm{Cl}\left(F_{+}\right)$denotes the closure of $F_{+}$, and $\tilde{\beta}=\nu \beta$ with $\nu=\operatorname{Ad}(g) \mid \mathfrak{h}_{\alpha}$ and $\beta=\nu_{\alpha} \alpha$.

1.5. Assume now that the center of $G$ is finite. An invariant eigendistribution $\pi$ on $G$ is tempered (for the definition, see [2(c), II, p. 20]), if and only if the function $\tilde{\kappa}^{\natural}$ on $H^{\mathfrak{y}}$ defined from $\pi$ by (1.3) satisfies the following condition for every $\mathfrak{h}$ [loc. cit., p. 38, Th. 7]: for any fixed element $h \in H^{\mathfrak{h}}$, there exists a polynomial function $P$ on $\mathfrak{h}$ satisfying

$$
\left|\tilde{\kappa}^{\mathfrak{h}}(h \exp X)\right| \leqslant P(X)
$$

for all $X \in \mathfrak{h}$ such that $h \exp X \in H^{\prime h}$. Moreover, when the infinitesimal character of $\pi$ is regular (for the definitions, see [4(c), $\S 2.4]$ ), this condition is equivalent to the following (cf. [loc. cit., Lem. 2.7]) :

(T) the function $\tilde{\kappa}^{\mathfrak{G}}$ is bounded on $H^{\prime \mathfrak{h}}$ for any $\mathfrak{h}$.

In the previous paper [4(d), §§8-9], we studied the existence and the uniqueness of tempered invariant eigendistributions on $G$ by a method of constructing the system of functions $\left\{\tilde{\kappa}^{\mathfrak{\xi}}\right\}$ inductively according to the order $\prec$ in $\operatorname{Car}^{0}(G)$. In this paper we are mainly concerned with the characters of discrete series representations.

By definition, an irreducible unitary representation of $G$ belongs to the discrete series if it is square-integrable. A group $G$ has a discrete series representation if and only if it has a compact Cartan subgroup $B$ [2(c), II]. In that case, $B$ is connected and its conjugate class $[B]$ is the unique maximal element in $\operatorname{Car}(G)$ and also in $\operatorname{Car}^{0}(G)$. Let $\mathfrak{b}$ the Cartan subalgebra corresponding to $B$ and $A$ a regular linear form on $\mathfrak{b}_{c}$ such that $B \ni \exp X \rightarrow$ $\exp A(X)(X \in \mathfrak{b})$ defines a character of $B$. Put

$$
\tilde{\kappa}^{\mathfrak{b}}(\exp X)=\sum_{w \in W_{G}(\mathfrak{b})} \operatorname{sgn}(w) e^{\Lambda(w X)} .
$$


THEOREM B [2(c), I, p.289, Th. 3]. There exists a tempered invariant eigendistribution $\Theta_{A}$ on $G$ such that $\Theta_{A}(h)=\Delta^{b}(h)^{-1} \tilde{\kappa}^{b}(h)\left(h \in B \cap G^{\prime}\right)$. Moreover such a distribution is unique.

Put

$$
q=\frac{1}{2}(\operatorname{dim} G-\operatorname{dim} K), \quad \varepsilon(\Lambda)=\operatorname{sgn}\left\{\prod_{\alpha \in P(\mathfrak{b})}\langle\Lambda, \alpha\rangle\right\},
$$

where $K$ is a maximal compact subgroup of $G$, and $\langle\Lambda, \alpha\rangle=\Lambda\left(H_{\alpha}\right)$. Then he also proved that the character of a representation in the discrete series is given in the form

$$
\pi_{\Lambda}=(-1)^{q} \varepsilon(\Lambda) \Theta_{\Lambda}
$$

for some $\Lambda$, and vice versa [2(c), II, § 41].

1.6. Now let $G=S p(n, \boldsymbol{R})$ for $n \geqslant 2$. We apply Theorem 1 and the condition ( $\mathrm{T}$ ) to compute the explicit forms of the discrete series representations. For $n=2,3$ and 4 , we compute the functions $\tilde{\kappa}_{j}^{r}$ 's inductively according to the order $\prec$ in $\operatorname{Car}^{0}(G)$ starting from $\tilde{\kappa}^{b}$ for the maximal element $[B]$ in it. The result for $n=2$ has been given in [4(b)].

In general, such inductive calculations determine uniquely $\tilde{\kappa}_{j}^{r}$ 's one by one (cf. [4(d), § 9]). But, for large $n$, these calculations can not be completed directly because of the extreme complexities both of the order in $\operatorname{Car}^{0}(G)$ and of the explicit forms of $\tilde{\kappa}_{j}^{r}$ 's. Thus we are obliged to use a heuristic method. This means the following. We presume some unified expression for each $\tilde{\kappa}_{j}^{r}$ available for any $\Lambda$. Then we test whether these presumed formulas for $\tilde{\kappa}_{j}^{r}$ 's satisfy or not the condition (T) and those (i),(ii) and (iii) in Theorem 1. Carefully taking into account the calculated results for $n=2,3$ and 4 , we succeeded to obtain fairly simple formulas for $\tilde{\kappa}_{j}^{r}$ 's as is presented in the following.

In this paper we will give in $\S 7$ the unified formulas for $\tilde{\kappa}_{j}^{r}$ s, and prove in $\S 8$ that they satisfy the conditions (T), (i), (ii) and (iii).

\section{§ 2. Structures of Cartan subgroups and Weyl groups}

2.1. Put

$$
H_{n}=\left(\begin{array}{cc}
0_{n} & 1_{n} \\
-1_{n} & 0_{n}
\end{array}\right), \quad I_{n}=\left(\begin{array}{cc}
1_{n} & 0_{n} \\
0_{n} & -1_{n}
\end{array}\right),
$$

where $1_{n}$ and $0_{n}$ denote the identity matrix and the zero matrix of order $n$ respectively. The group $S p(n, \boldsymbol{R})$ or $S p(n, \boldsymbol{C})$ is defined respectively as the 
group of real or complex matrices of order $2 n$ satisfying

$$
{ }^{t} g H_{n} g=H_{n},
$$

where ${ }^{t} g$ denotes the transposed matrix of $g$. We study the real form $S p(n, \boldsymbol{R})$ of $S p(n, C)$. But, for later conveniences, we always treat in this paper the following group isomorphic to $S p(n, \boldsymbol{R})$ :

$$
G_{n}=\left\{g \in S p(n, C) ; g^{*} I_{n} g=I_{n}\right\}
$$

where $g^{*}={ }^{t} \bar{g}$.

Put $G=G_{n}$ and let $\mathrm{g}$ be its Lie algebra. Let $\theta$ be the Cartan involution of $\mathfrak{g}$ given by $\theta X=I_{n} X I_{n}(X \in \mathfrak{g})$, and $\mathfrak{g}=\mathfrak{f}+\mathfrak{p}$ the corresponding decomposition of $\mathfrak{g}$. Then the maximal compact subgroup $K$ corresponding to $\mathfrak{f}$ is given as

$$
K=\left\{\left(\begin{array}{cc}
u & 0_{n} \\
0_{n} & \bar{u}
\end{array}\right) ; u \in U(n)\right\}
$$

where $U(n)$ denotes the group of all unitary matrices of order $n$.

2.2. Let $k, l$ and $m$ be non-negative integers such that

$$
k+2 l+m=n \text {. }
$$

Let $\mathfrak{h}^{k, l}$ be a $\theta$-invariant Cartan subalgebra of $\mathfrak{g}$ given as $\mathfrak{h}^{k, l}=\mathfrak{h}_{-}^{k, l}+\mathfrak{h}_{+}^{k, l}$, where $\mathfrak{h}_{-}^{k, l}=\mathfrak{h}^{k, l} \cap \mathfrak{p}$ and $\mathfrak{h}_{+}^{k, l}=\mathfrak{h}^{k, l} \cap \mathfrak{l}$ consist of the elements of the following forms respectively:

$$
\mathfrak{h}_{-}^{k, l}: \quad X_{-}=\left(\begin{array}{ccccccc}
0_{k} & & & 0_{k} & & \\
& 0_{2 l} & & & A & \\
& & 0_{m} & & & B \\
0_{k} & & & 0_{k} & & \\
& A & & & 0_{2 l} & \\
& & B & & & 0_{m}
\end{array}\right)
$$

with

$$
A=\left(\begin{array}{cccc}
\tau_{1} j_{2} & & & \\
& \tau_{2} j_{2} & & \\
& & \ddots & \\
& & & \tau_{l} j_{2}
\end{array}\right), \quad B=\left(\begin{array}{llll}
t_{1} & & & \\
& t_{2} & & \\
& & \ddots & \\
& & & t_{m}
\end{array}\right), \quad j_{2}=\left(\begin{array}{ll}
0 & 1 \\
1 & 0
\end{array}\right)
$$




$$
\begin{aligned}
\mathfrak{H}_{+}^{k, l}: & X_{+}= \\
& \operatorname{diag}\left(i \varphi_{1}, i \varphi_{2}, \cdots, i \varphi_{k} ; i \theta_{1},-i \theta_{1}, \cdots, i \theta_{l},-i \theta_{l} ; 0,0, \cdots, 0 ;\right. \\
& \left.-i \varphi_{1},-i \varphi_{2}, \cdots,-i \varphi_{k} ;-i \theta_{1}, i \theta_{1}, \cdots,-i \theta_{l}, i \theta_{l} ; 0,0, \cdots, 0\right),
\end{aligned}
$$

where $\tau_{p}, t_{q}, \varphi_{r}, \theta_{s} \in R, i=\sqrt{-1}$, the blank spaces must be filled with zeros and diag $\left(a_{1}, a_{2}, \cdots, a_{2 n}\right)$ denotes a diagonal matrix with diagonal elements $a_{1}, a_{2}, \cdots, a_{2 n}$.

Then $\left\{\mathfrak{h}^{k, l} ; k+2 l \leqslant n\right\}$ forms a complete system of representatives of the conjugate classes in Car (g) (cf. [6, pp. 410-411]).

Put $X=X_{-}+X_{+}$and arrange the eigenvalues of $X$ as

$$
\left(x_{1}, x_{2}, \cdots, x_{n} ;-x_{1},-x_{2}, \cdots,-x_{n}\right),
$$

where

$$
\begin{cases}x_{p}=i \varphi_{p} & (1 \leqslant p \leqslant k) ; \\ x_{k+2 q-1}=z_{q}=\tau_{q}+i \theta_{q}, x_{k+2 q}=\bar{z}_{q}=\tau_{q}-i \theta_{q} & (1 \leqslant q \leqslant l) ; \\ x_{k+2 l+r}=t_{r} & (1 \leqslant r \leqslant m) .\end{cases}
$$

We use $x=\left(x_{1}, x_{2}, \cdots, x_{n}\right)$ as the co-ordinates of $X \in \mathfrak{h}^{k, l}$.

Fix an order of roots of $\mathfrak{h}^{k, l}$ in such a way that the set of positive roots $P\left(\mathfrak{h}^{k, l}\right)$ is given by

$$
\alpha(X)=x_{p} \pm x_{q} \quad(1 \leqslant p<q \leqslant n), \quad 2 x_{p} \quad(1 \leqslant p \leqslant n) .
$$

Then the set of real positive $\operatorname{roots} S_{R}\left(\mathfrak{h}^{k, l}\right)$ is given by

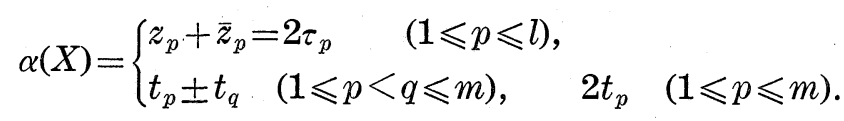

An imaginary root $\alpha$ is called compact or singular if its root vector $X_{\alpha}$ belongs to $\mathfrak{f}_{c}$ or $\mathfrak{p}_{c}$ respectively. Denote by $P_{0}\left(\mathfrak{h}^{k, l}\right)$ and $S_{I}\left(\mathfrak{h}^{k, l}\right)$ the sets of positive compact or singular imaginary roots respectively. Then they are respectively given as

$$
\begin{aligned}
& P_{0}\left(\mathfrak{h}^{k, l}\right): \alpha(X)=i \varphi_{p}-i \varphi_{q} \quad(1 \leqslant p<q \leqslant k) ;
\end{aligned}
$$

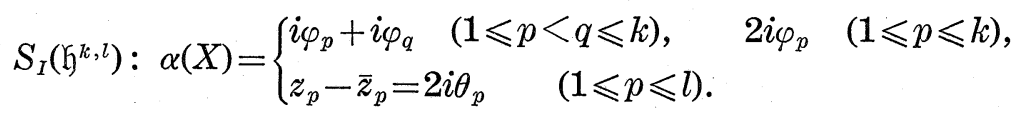

2.3. Let $H^{k, l}$ be the Cartan subgroup of $G$ corresponding to $\mathfrak{h}^{k, l}$. Then

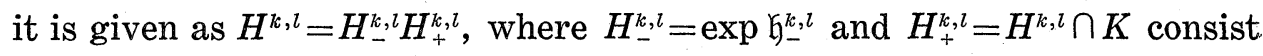
of the elements of the following forms respectively: 


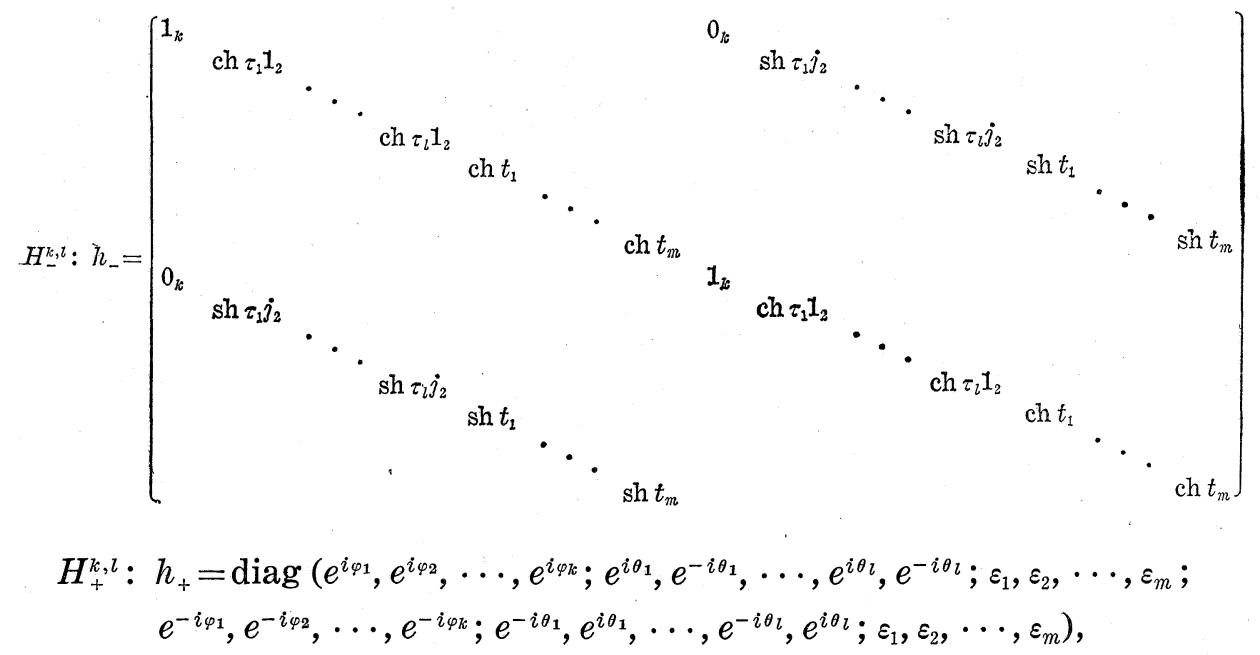

where $i=\sqrt{-1}$ and $\varepsilon_{p}= \pm 1$. Then $\left\{H^{k, l} ; k+2 l \leqslant n\right\}$ is a complete system of representatives of the conjugate classes in $\operatorname{Car}(G)$.

Put $h=h_{-} h_{+}$and arrange its eigenvalues as

$$
\left(\delta_{1}, \delta_{2}, \cdots, \delta_{n} ; \delta_{1}^{-1}, \delta_{2}^{-1}, \cdots, \delta_{n}^{-1}\right),
$$

where

$$
\begin{cases}\delta_{p}=e^{i \varphi_{p}} & (1 \leqslant p \leqslant k) ; \\ \delta_{k+2 q-1}=e^{z_{q}}, \quad \delta_{k+2 q}=e^{\bar{z}_{q}} & (1 \leqslant q \leqslant l) ; \\ \delta_{k+2 l+r}=\varepsilon_{r} e^{t_{r}} & (1 \leqslant r \leqslant m) .\end{cases}
$$

We use $\delta=\left(\delta_{1}, \delta_{2}, \cdots, \delta_{n}\right)$ as the co-ordinates of $h \in H^{k, l}$.

The set of characters $\xi_{\alpha}$ of $H^{k, l}$ corresponding to $\alpha \in P\left(\mathfrak{h}^{k, l}\right)$ are given as

$$
\xi_{\alpha}(h)=\delta_{p} \delta_{q}, \delta_{p} \delta_{q}^{-1} \quad(1 \leqslant p<q \leqslant n), \quad \delta_{p}^{2} \quad(1 \leqslant p \leqslant n) .
$$

Put $\Delta^{k, l}=\Delta^{\mathfrak{h}}, \Delta_{R}^{\prime k, l}=\Delta_{R}^{\prime \mathfrak{h}}$ for $\mathfrak{h}=\mathfrak{h}^{k, l}$. Then

$$
\begin{aligned}
\Delta^{k, l}(h)= & \prod_{1 \leqslant p<q \leqslant n}\left\{\left(\delta_{p}+\delta_{p}^{-1}\right)-\left(\delta_{q}+\delta_{q}^{-1}\right)\right\} \cdot \prod_{1 \leqslant p \leqslant n}\left(\delta_{p}-\delta_{p}^{-1}\right), \\
\Delta_{R}^{\prime k, l}(h)= & \prod_{1 \leqslant p \leqslant l}\left(1-e^{-2 \tau_{p}}\right) \cdot \prod_{1 \leqslant p \leqslant m}\left(1-e^{-2 t_{p}}\right) \\
& \times \prod_{1 \leqslant p<q \leqslant m}\left\{\left(1-\varepsilon_{p} \varepsilon_{q} e^{-t_{p}-t_{q}}\right)\left(1-\varepsilon_{p} \varepsilon_{q} e^{-t_{p}+t_{q}}\right)\right\} .
\end{aligned}
$$

2.4. Let us determine the group $W_{G}\left(H^{k, l}\right)$. For any real root $\alpha \in S_{R}\left(\mathfrak{h}^{k, l}\right)$, the element $\omega_{\alpha}$ in (1.14) belongs to $W_{G}\left(H^{k, l}\right)$. For any compact root $\alpha \in P_{0}\left(\mathfrak{h}^{k, l}\right)$, we can choose root vectors $X_{ \pm \alpha}$ in such a way that $X_{\alpha}-X_{-\alpha}$ $\in \mathfrak{f}$. Then the following element $\omega_{\alpha}$ also belongs to $W_{G}\left(H^{k, l}\right)$ : 


$$
\omega_{\alpha} h=g_{\alpha} h g_{\alpha}^{-1} \quad\left(h \in H^{k, l}\right),
$$

where $g_{\alpha}=\exp \frac{\pi}{2}\left(X_{\alpha}^{\prime}-X_{-\alpha}^{\prime}\right) \in K$

To determine $W_{G}\left(H^{k, l}\right)$, we apply the following lemma.

LEMMA 2.1. For $G=G_{n}$, the group $W_{G}\left(H^{k, l}\right)$ is canonically isomorphic to $W_{G}\left(\mathfrak{h}^{k, l}\right)$ under the homomorphism $\omega \rightarrow \bar{\omega}$. Let $N_{K}\left(\mathfrak{h}^{k, l}\right)$ and $Z_{K}\left(\mathfrak{h}^{k, l}\right)$ be the normalizer and the centralizer of $\mathfrak{h}^{k, l}$ in $K$ respectively. Then $W_{G}\left(\mathfrak{h}^{k, l}\right)$ is canonically isomorphic to $N_{K}\left(\mathfrak{h}^{k, l}\right) / Z_{K}\left(\mathfrak{h}^{k, l}\right)$ and $Z_{K}\left(\mathfrak{h}^{k, l}\right)=H^{k, l} \cap K=H_{+}^{k, l}$.

Proof. In general, the kernel of the homomorphism $\omega \rightarrow \bar{\omega}$ of $W_{G}\left(H^{\mathfrak{b}}\right)$ onto $W_{G}(\mathfrak{h})$ is $H^{\mathfrak{G}} / H_{0}^{\mathfrak{\zeta}}$, where $H_{0}^{\mathfrak{\zeta}}$ denotes the center of $H^{\mathfrak{h}}$. In the present case, every Cartan subgroup is commutative and hence the first assertion. Since $\mathfrak{h}^{k, l}$ is $\theta$-invariant, the second assertion follows from [2(b), $\S 16$, Cor. 2 of Lem. 26].

Q.E.D.

We see without difficulty that $W_{G}\left(H^{k, l}\right)$ contains the elements $\omega$ listed below. Here we use the co-ordinates $\delta=\left(\delta_{1}, \delta_{2}, \cdots, \delta_{n}\right)$ in (2.9) of $h \in H^{k, l}$, and denote by $\widetilde{\varsigma}_{k}$ the symmetric group of order $k$.

\begin{tabular}{c|c} 
operation of $\omega \in W_{G}\left(H^{k, l}\right)$ & $\tilde{\varepsilon}(\omega)$ \\
\hline any permutation $w \in \mathbb{S}_{k}$ of $e^{i \varphi_{1}}, e^{i \varphi_{2}}, \cdots, e^{i \varphi_{k}}$ & $\operatorname{sgn}(w)$ \\
any permutation of $z_{1}, z_{2}, \cdots, z_{l}$ or of the pairs & 1 \\
$\quad\left(\tau_{1}, \theta_{1}\right),\left(\tau_{2}, \theta_{2}\right), \cdots,\left(\tau_{l}, \theta_{l}\right)$ & -1 \\
change of the sign of $\tau_{r}(1 \leqslant r \leqslant l):$ & -1 \\
$\quad \tau_{r} \rightarrow-\tau_{r}\left(\right.$ or $\left.z_{r} \rightarrow-\bar{z}_{r}\right)$ & -1 \\
change of the sign of $\theta_{r}(1 \leqslant r \leqslant l):$ & $\operatorname{sgn}(w)$ \\
$\quad \theta_{r} \rightarrow-\theta_{r}$ (or $\left.z_{r} \rightarrow \bar{z}_{r}\right)$ & -1 \\
any permutation $w \in \mathbb{S}_{m}$ of $\varepsilon_{1} e^{t_{1}}, \varepsilon_{2} e^{t_{2}}, \cdots, \varepsilon_{m} e^{t_{m}}$ & \\
change of the sign of $t_{r}(1 \leqslant r \leqslant m): t_{r} \rightarrow-t_{r}$ &
\end{tabular}

Moreover we get the following theorem.

THEOREM 2. For $G=G_{n}$, the group $W_{G}\left(H^{k, l}\right)$ is generated by the elements listed above.

Proof. By Lemma 2.1 and the explicit form of the subgroup $K$ in (2.4), this can be proved without difficulty.

2.5. Put $\varepsilon=\left(\varepsilon_{1}, \varepsilon_{2}, \cdots, \varepsilon_{m}\right)$, where $\varepsilon_{r}= \pm 1$. Then it determines a connected component $H^{k, l}(\varepsilon)$ of $H^{k, l}$ consisting of such elements $h=h_{-} h_{+}$that $h_{+}$contains $\varepsilon$ just as in (2.9). Thus the number of connected components of $H^{k, l}$ is equal to $2^{m}$, where $m=n-k-2 l$. For $0 \leqslant j \leqslant m$, let $H_{j}^{k, l}$ be the 
connected component $H^{k, l}(\varepsilon)$ for which $\varepsilon_{1}=\varepsilon_{2}=\cdots=\varepsilon_{j}=1, \varepsilon_{j+1}=\cdots=\varepsilon_{m}=-1$. Then we get the following

THEOREM 3. For $G=G_{n} \cong S p(n, R)$, a complete system of representatives of the conjugate classes in $\operatorname{Car}^{0}(G)$ is given by

$$
\left\{H_{j}^{k, l} ; k+2 l \leqslant n, 0 \leqslant j \leqslant m\right\} .
$$

Proof. This follows easily from the structure of $W_{G}\left(H^{k, l}\right)$ given above.

Q.E.D.

2.6. The set of real roots $\alpha \in S_{R}\left(\mathfrak{h}^{k, l}\right)$ such that $\xi_{\alpha}(h)>0$ for $h \in H_{j}^{k, l}$ is given as

$$
\xi_{\alpha}(h)= \begin{cases}e^{2 \tau_{r}}=e^{z_{r}} e^{\bar{z}_{r}} & \quad(1 \leqslant r \leqslant l), \\ e^{t_{p \pm} t_{q}} & (1 \leqslant p<q \leqslant j \text { or } j+1 \leqslant p<q \leqslant m), \\ e^{2 t_{p}} & (1 \leqslant p \leqslant m) .\end{cases}
$$

The connected component $F_{+}$of $H_{j}^{k, l} \cap H^{\prime k, l}(R)$ is by definition given by $F_{+}$ $=\left\{h \in H_{j}^{k, l} ; 1-\xi_{\alpha}(h)^{-1}>0\left(\alpha \in S_{R}\left(\mathfrak{G}^{k, l}\right)\right)\right\}$. Therefore it is determined by the following conditions on $h \in H_{j}^{k, l}$ :

$$
\left\{\begin{array}{l}
\tau_{r}>0 \quad(1 \leqslant r \leqslant l), \\
t_{1}>t_{2}>\ldots>t_{j}>0, \quad t_{j+1}>t_{j+2}>\cdots>t_{m}>0 .
\end{array}\right.
$$

Hence the set $\Pi\left(F_{+}\right)$of $\alpha \in S_{R}\left(\mathfrak{h}^{k, l}\right)$ giving the walls of $F_{+}$by $\xi_{\alpha}(h)=1$ is given as

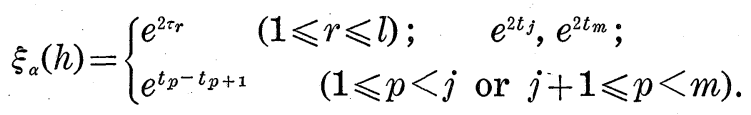

We see from the results in $\S 2.4$ that the group $W_{G}\left(F_{+}\right)$for $F_{+} \subset H_{j}^{k, l}$ is generated by the elements listed below.

\begin{tabular}{l|c} 
operation of $\omega \in W_{G}\left(F_{+}\right)$ & $\tilde{\varepsilon}(\omega)$ \\
\hline any permutation $w \in \Im_{k}$ of $e^{i \varphi_{1}}, e^{i \varphi_{2}}, \cdots, e^{i \varphi_{k}}$ & $\operatorname{sgn}(w)$ \\
any permutation of $z_{1}, z_{2}, \cdots, z_{l}$ & 1 \\
change of the sign of $\theta_{r}(1 \leqslant r \leqslant l)$ & -1
\end{tabular}

\section{§3. Orders in $\operatorname{Car}(G)$ and $\operatorname{Car}^{0}(G)$}

3.1. Let us determine the order $<$ in $\operatorname{Car}(G)$. Recall that for a Cartan subalgebra $\mathfrak{h}$ of $\mathfrak{g}$ and a real root $\alpha$ of $\mathfrak{h}$, we define in $\S 1.3$ a Cartan subalgebra $\mathfrak{h}_{\alpha}$ and write this relation as $\mathfrak{h}^{\stackrel{\alpha}{\longrightarrow}} \mathfrak{h}_{\alpha}$. Moreover the relation be- 
tween the conjugate classes [h] and $\left[\mathfrak{h}_{\alpha}\right]$ is denoted also by $[\mathfrak{h}] \stackrel{\alpha}{\longrightarrow}\left[\mathfrak{h}_{\alpha}\right]$.

Consider the real roots of $\mathfrak{h}^{k, l}$ given in (2.8). Then, representing $\alpha$ by $\alpha(X)$, we see that the positive real roots are divided into three conjugate classes under $W_{G}\left(\mathfrak{h}^{k, l}\right)$ as follows:

(1) the roots $2 \tau_{p} \quad(1 \leqslant p \leqslant l)$;

(2) the roots $2 t_{p} \quad(1 \leqslant p \leqslant m)$;

(3) the roots $t_{p} \pm t_{q} \quad(1 \leqslant p<q \leqslant m)$.

Hence we get without difficulty the next lemma.

LEMMA 3.1. There hold the following relations and moreover they determine the order < in Car (g) completely:

$$
\begin{array}{ll}
{\left[\mathfrak{h}^{k, l}\right] \stackrel{\alpha}{\longrightarrow}\left[\mathfrak{h}^{k+2, l-1}\right]} & \text { for any } \alpha \text { in }(1) ; \\
{\left[\mathfrak{h}^{k, l}\right] \stackrel{\alpha}{\longrightarrow}\left[\mathfrak{h}^{k+1, l}\right]} & \text { for any } \alpha \text { in }(2) ; \\
{\left[\mathfrak{h}^{k, l}\right] \stackrel{\alpha}{\longrightarrow}\left[\mathfrak{h}^{k, l+1}\right]} & \text { for any } \alpha \text { in }(3) .
\end{array}
$$

The proof of this lemma is omitted here.

The order $<$ in $\operatorname{Car}(G)$ is by definition the direct translation of that in $\operatorname{Car}(\mathfrak{g})$. For later conveniences, we illustrate it for $G=G_{5} \cong S p(5, R)$, representing $\left[H^{k, l}\right]$ by the point $(l, k+l)$.

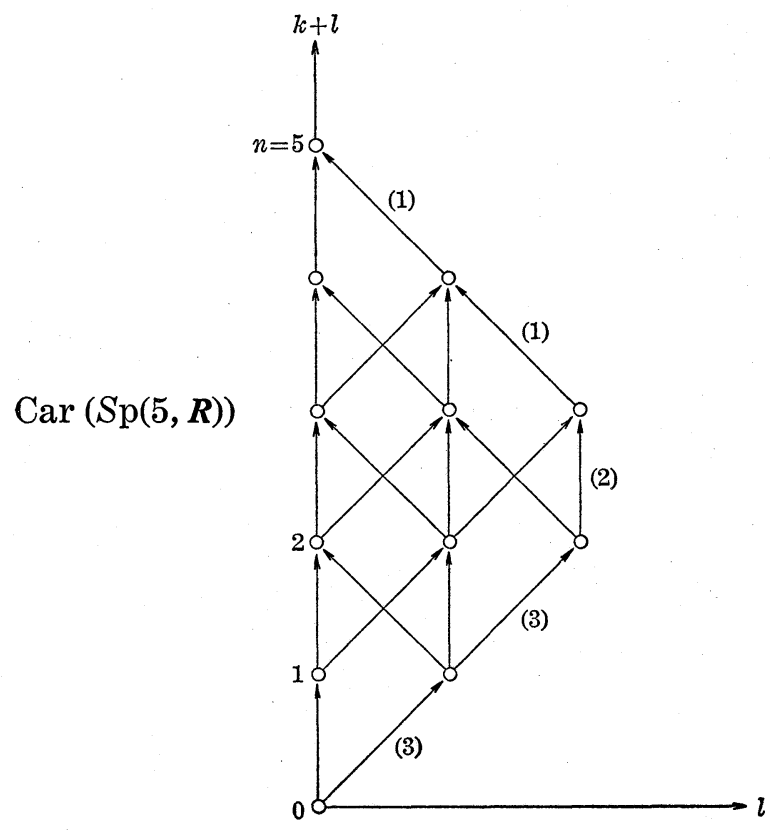

Figure 3.1. 
3.2. Let us determine the order $\prec$ in $\operatorname{Car}^{0}(G)$. Fix any $H_{j}^{k, l}$. Let $P^{\prime}$ be the set of positive real roots $\alpha \in S_{R}\left(\mathfrak{h}^{k, l}\right)$ such that $\xi_{\alpha}(h)>0$ for $h \in H_{j}^{k, l}$, and $W^{\prime}$ be the subgroup of $W_{G}\left(H^{k, l}\right)$ generated by the set $\left\{\omega_{\alpha} ; \alpha \in P^{\prime}\right\}$. Note that $\bar{\omega}_{\alpha}=s_{\alpha}$, the reflexion corresponding to the root $\alpha$. Then we see that $\bar{W}^{\prime}=\left\{\bar{\omega} ; \omega \in W^{\prime}\right\}$ is generated by $\left\{s_{\alpha} ; \alpha \in P^{\prime}\right\}$ and that every root $\alpha^{\prime}$ in $P^{\prime}$ is conjugate under $W^{\prime}$ to some root $\alpha$ in $\Pi\left(F_{+}\right)$with $F_{+} \subset H_{j}^{k, l}$. This means that, to determine the order $\prec$ in $\operatorname{Car}^{0}(G)$, it is sufficient to consider the relations $\left[H_{j}^{k, l}\right] \stackrel{\alpha}{\longrightarrow}[\cdot]$ only for $\alpha \in \Pi\left(F_{+}\right)$. This, in turn, is the another expression of the fact that in the equations (1.20) in Theorem 1, it is sufficient to treat only those real roots $\alpha$ which belong to $\Pi\left(F_{+}\right)$.

LEMMA 3.2. There hold the following relations and moreover they determine the order $\prec$ in $\operatorname{Car}^{0}(G)$ completely:

(1) $\left[H_{j}^{k, l}\right] \stackrel{\alpha}{\longrightarrow}\left[H_{j}^{k+2, l-1}\right]$, where $\alpha(X)=2 \tau_{p}(1 \leqslant p \leqslant l)$;

$\left(2^{+}\right) \quad\left[H_{j}^{k, l}\right] \stackrel{\alpha}{\longrightarrow}\left[H_{j-1}^{k+1, l}\right]$, where $\alpha(X)=2 t_{j}$;

(2- $\left[H_{j}^{k, l}\right] \stackrel{\alpha}{\longrightarrow}\left[H_{j}^{k+1, l}\right]$, where $\alpha(X)=2 t_{m}$;

$\left(3^{+}\right)\left[H_{j}^{k, l}\right] \stackrel{\alpha}{\longrightarrow}\left[H_{j-2}^{k, l+1}\right]$, where $\alpha(X)=t_{p}-t_{p+1}(1 \leqslant p \leqslant j-1)$;

(3-) $\left[H_{j}^{k, l}\right] \stackrel{\alpha}{\longrightarrow}\left[H_{j}^{k, l+1}\right]$, where $\alpha(X)=t_{p}-t_{p+1}(j+1 \leqslant p \leqslant m-1)$.

Here, for example, $\left(3^{+}\right)$occurs when $k+2(l+1) \leqslant n$ and $j \geqslant 2$.

Proof. We prove the lemma using the explicit forms of $H_{j}^{k, l}$ 's in $\S 2.5$ and those of $W_{G}\left(H_{j}^{k, l}\right)$ in $\S 2.4$. The detailed calculations are omitted here but will be found in the course of the next section.

Q.E.D.

Note that when $k+2 l=n$ (i.e., $m=0$ ), $H^{k, l}$ is connected and $H_{0}^{k, l}=H^{k, l}$. For later conveniences, we illustrate the orders in $\operatorname{Car}^{0}(G)$ for $G=G_{2} \cong$ $S p(2, R)$ and $G_{3} \cong S p(3, R)$, omitting the blackets for simplicity. 

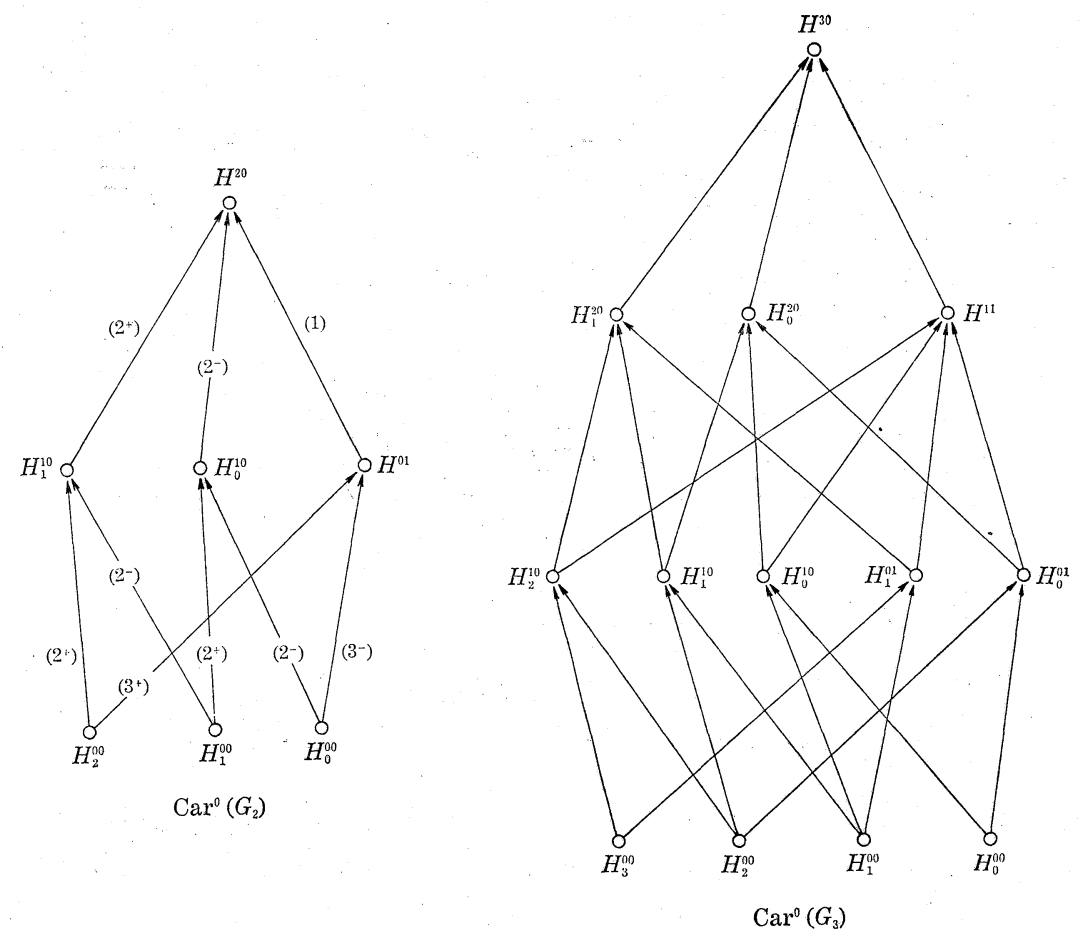

Figure 3.2.

\section{§4. Differential equations connecting $\tilde{\kappa}^{\natural}$ and $\tilde{\kappa}^{\text {bx }}$}

4.1. Let us write down explicitly the equations (1.20) in Theorem 1 with respect to the co-ordinates introduced in $\S 2.3$. Put $\tilde{\kappa}^{k, l}=\tilde{\kappa}^{\mathfrak{l}}$ for $\mathfrak{h}=\mathfrak{h}^{k, l}$ and $\tilde{\kappa}_{j}^{k, l}=\tilde{\kappa}^{k, l} \mid F_{+}$for $F_{+} \subset H_{j}^{k, l}$. Then, according to Lemma 3.2, these equations are divided into five cases $(1),\left(2^{+}\right),\left(2^{-}\right),\left(3^{+}\right)$and $\left(3^{-}\right)$, and in each case, they connect the analytic functions $\tilde{\kappa}_{j}^{k, l}$ with such a one corresponding to the end of the arrow in the following figure (the blackets are omitted here):

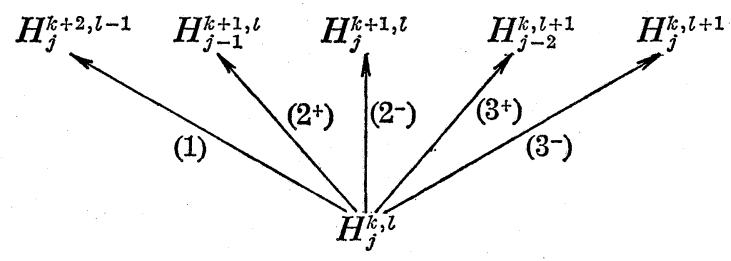

We introduce the following notations:

$$
\Phi_{p}=i \varphi_{p}, \quad \Theta_{q}=i \theta_{q}, \quad \frac{\partial}{\partial \Phi_{p}}=\frac{1}{i} \frac{\partial}{\partial \varphi_{p}}, \quad \frac{\partial}{\partial \Theta_{q}}=\frac{1}{i} \frac{\partial}{\partial \theta_{q}} \quad(i=\sqrt{-1}) .
$$


4.2. Case (1). The roots $\alpha$ in $\Pi\left(F^{+}\right)$corresponding to this case are given by $\alpha(X)=2 \tau_{p}(1 \leqslant p \leqslant l)$. For every such $\alpha$, there exists some $g \in G$ such that $H_{j}^{k, l} \stackrel{\alpha, g}{\longrightarrow} H_{j}^{k+2, l-1}$ or $H_{j}^{k, l} \stackrel{\alpha}{\longrightarrow} g^{-1} H_{j}^{k+2, l-1} g$. This means by definition that $\left(H_{j}^{k, l} \cap \Sigma_{\alpha}^{\prime}\right) \cap g^{-1} H_{j}^{k+2, l-1} g \neq \varnothing$. We must calculate $H_{\alpha}^{\prime}, H_{\beta}^{\prime}=\sqrt{-1}\left(X_{\alpha}^{\prime}-X_{-\alpha}^{\prime}\right)$ in $\S 1.2$ and $w \in W\left(\mathfrak{h}_{c}^{k+2, l-1}\right)$ in (1.18).

Denote by $g_{n}$ the Lie algebra of $G_{n}$. Let us first consider the case $n=2$. An appropriate choice of the root vectors $X_{ \pm \alpha}$ from $g_{2} \cong g_{p}(2, R)$ gives us by (1.6) the following:

$$
H_{\alpha}^{\prime}=\left(\begin{array}{ll}
0_{2} & j_{2} \\
j_{2} & 0_{2}
\end{array}\right), \quad X_{\varepsilon \alpha}^{\prime}=\frac{\sqrt{-1}}{2}\left(\begin{array}{cc}
-\varepsilon 1_{2} & j_{2} \\
-j_{2} & \varepsilon 1_{2}
\end{array}\right) \quad(\varepsilon= \pm 1)
$$

where $j_{2}=\left(\begin{array}{ll}0 & 1 \\ 1 & 0\end{array}\right)$. Hence

$$
H_{\beta}^{\prime}=\left(\begin{array}{cc}
1_{2} & 0_{2} \\
0_{2} & -1_{2}
\end{array}\right)=\frac{1}{\sqrt{-1}}\left(\begin{array}{cccc}
\sqrt{-1} & 1_{2} & 0_{2} \\
0_{2} & -\sqrt{-1} & 1_{2}
\end{array}\right) .
$$

In the general case of $n$, take $\alpha(X)=2 \tau_{p}$ for $1 \leqslant p \leqslant l$. Consider the isomorphism $\phi$ of $g_{2}$ into $g_{n}$ defined as follows: for an element $Y=\left(y_{r s}\right)_{1 \leqslant r, s \leqslant 4}$ in $g_{2}$, we make correspond $X=\left(x_{r s}\right)_{1 \leqslant r, s \leqslant 2 n}$ in $g_{n}$ such that

$$
\left(x_{r s}\right)_{r, s=k+2 p-1, k+2 p, n+k+2 p-1, n+k+2 p}=Y
$$

and all the other components $x_{r s}$ 's are zero. Then, taking into account (1.9), we see that the images by $\phi$ of the elements of $g_{2}$ in (4.2) can be taken as the corresponding elements for $\mathfrak{g}_{n}$. Hence we denote $\phi\left(H_{\alpha}^{\prime}\right), \phi\left(X_{ \pm \alpha}^{\prime}\right)$ and $\phi\left(\sqrt{-1} H_{\beta}^{\prime}\right)$ again by $H_{\alpha}^{\prime}, X_{ \pm \alpha}^{\prime}$ and $\sqrt{-1} H_{\beta}^{\prime}$ respectively.

Put $g \in G$ as

$$
\mathrm{g}=\left(\begin{array}{ccccccc}
1_{k} & & & & & & \\
& 0 & 1_{2} & & & & \\
& 1_{2 p-2} & 0 & & & & \\
& & & 1_{n-2 p} & & & \\
& & & & 0 & 1_{2} & \\
& & & & 1_{2 p-2} & 0 & \\
& & & & & & 1_{n-k-2 p}
\end{array}\right) .
$$

Then we get Ad $(g) \mathfrak{h}_{\alpha}=\mathfrak{h}^{k+2, l-1}$ with $\mathfrak{h}=\mathfrak{h}^{k, l}$, and $H_{j}^{k, l} \stackrel{\alpha, g}{\longrightarrow} H_{j}^{k+2, l-1}$.

Let $D \in S\left(\mathfrak{h}_{c}\right)$. Here we denote by $\partial(D)$ the left invariant differential operator on $H^{\natural}$ corresponding canonically to $D$. We use the co-ordinates on $H^{k, l}$ given by (2.9) and those on $H^{k+2, l-1}$ given analogously. Put on $H^{k+2, l-1}$, 


$$
\begin{cases}\psi=\varphi_{k+1}+\varphi_{k+2}, & \Psi=i \psi, \\ \theta=\varphi_{k+1}-\varphi_{k+2}, & \Theta=i \theta \quad(i=\sqrt{-1})\end{cases}
$$

then we get

$$
\begin{array}{ll}
\partial\left(H_{\alpha}^{\prime}\right)=\frac{\partial}{\partial \tau_{p}} & \text { on } H^{k, l}, \\
\partial\left(H_{\tilde{\beta}}^{\prime}\right)=\partial\left(\operatorname{Ad}(g) H_{\beta}^{\prime}\right)=\frac{\partial}{\partial \Psi} & \text { on } H^{k+2, l-1},
\end{array}
$$

where $\tilde{\beta}=\nu \beta$ with $\nu=\operatorname{Ad}(g) \mid \mathfrak{h}_{\alpha}, \mathfrak{h}=\mathfrak{h}^{k, l}$, and $\partial / \partial \Psi=i^{-1} \partial / \partial \psi$.

Let us now determine $w \in W\left(\mathfrak{G}_{c}^{k+2, l-1}\right)$ in (1.18) or the unique element satisfying

$$
P\left(\mathfrak{h}_{\alpha}\right)^{\nu}=P\left(\mathfrak{h}^{k+2, l-1}\right)^{w} .
$$

The set of positive roots $P\left(\mathfrak{h}^{k+2, t-1}\right)$ is defined in $\S 2.2$ as follows. Introduce in $\mathfrak{h}^{k+2, l-1}$ the co-ordinates $\left(x_{1}, x_{2}, \cdots, x_{n}\right)$ analogously as (2.6) by

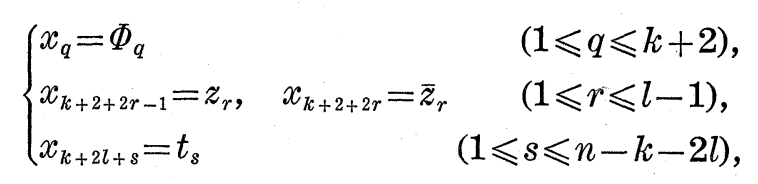

and let $X$ be the element in $\mathfrak{h}^{k+2, l-1}$ with these co-ordinates. Then

$$
P\left(\mathfrak{h}^{k+2, l-1}\right)=\left\{\alpha ; \alpha(X)=x_{q} \pm x_{r}(1 \leqslant q<r \leqslant n), 2 x_{q}(1 \leqslant q \leqslant n)\right\} .
$$

On the other hand, we see that $P\left(\mathfrak{h}_{\alpha}\right)^{\nu}$ is given as follows: put

$$
\begin{aligned}
\left(y_{1}, y_{2}, \cdots, y_{n}\right)= & \left(x_{1}, x_{2}, \cdots, x_{k}, \hat{x}_{k+1}, \hat{x}_{k+2},\right. \\
& x_{k+3}, x_{k+4}, \cdots, x_{k+2 p+1}, x_{k+2 p+2}, x_{k+1}, x_{k+2}, \\
& \left.x_{k+2 p+3}, x_{k+2 p+4}, \cdots, x_{n}\right),
\end{aligned}
$$

then,

$$
P\left(\mathfrak{h}_{\alpha}\right)^{\nu}=\left\{\alpha ; \alpha(X)=y_{q} \pm y_{r}(1 \leqslant q<r \leqslant n), 2 y_{q}(1 \leqslant q \leqslant n)\right\} .
$$

In other words, we get $P\left(\mathfrak{h}_{\alpha}\right)^{\nu}$ with $\mathfrak{h}=\mathfrak{h}^{k, l}, P\left(\mathfrak{h}_{\alpha}\right)=P(\mathfrak{h})^{\nu \alpha}$, from $P\left(\mathfrak{h}^{k, l}\right)$ by making the following replacements in the co-ordinates (2.6) in $\mathfrak{h}^{k, l}$ :

$$
\left\{\begin{array}{l}
z_{p}=\tau_{p}+\Theta_{p} \rightarrow \Phi_{k+1}=\Psi+\Theta, \\
\bar{z}_{p}=\tau_{p}-\Theta_{p} \rightarrow \Phi_{k+2}=\Psi-\Theta ; \\
z_{q} \rightarrow z_{q-1}, \quad \bar{z}_{q} \rightarrow \bar{z}_{q-1} \quad(p<q \leqslant l) .
\end{array}\right.
$$

Thus we see that (4.8) gives the co-ordinates of $w^{-1} X$, and so the permuta- 
tion in the right hand side of (4.8) gives the element: $w^{-1} \in W\left(\mathfrak{h}_{c}^{k+2, l-1}\right)$. Hence

$$
\operatorname{sgn}(w)=1
$$

The subsets $\Sigma_{\alpha}$ and $\Sigma_{\tilde{\beta}}$ of $H^{k, l}$ and $H^{k+2, l-1}$ are defined respectively by $e^{2 \tau}=1$ and $e^{2 \Psi}=e^{\Phi_{k+1}+\Phi_{k+2}}=1$, or rather by $\tau_{p}=0$ and $\Psi=0$. Moreover the replacements (4.9) of the co-ordinates, restricted to the case where $\tau_{p}=0$ and $\Psi=0$, gives us the co-ordinates of $h^{g}=g h g^{-1} \in \Sigma_{\tilde{\beta}} \subset H^{k+2, l-1}$ from those of $h \in \Sigma_{\alpha} \subset H^{k, l}$. (This replacement is essentially a chage of suffices of the co-ordinates.)

Consider $\tilde{\kappa}_{j}^{k, l}$ and $\tilde{\kappa}_{j}^{k+2, l-1}$ as the functions of the co-ordinates (2.6) and (4.7) respectively. Then it follows from (4.6), (4.10) and the above remark that the equations (1.20) for $H_{j}^{k, l} \stackrel{\alpha, g}{\longrightarrow} H_{j}^{k+2, l-1}$ are given as follows: for $M=$ $0,1,2, \cdots$,

$$
\begin{gathered}
\left.\left(\frac{\partial}{\partial \tau_{p}}\right)_{0}^{2 M+1} \tilde{\kappa}_{j}^{k, l}\right|_{(4.12)}=\left(\frac{\partial}{\partial \Psi}\right)_{0}^{2 M+1} \tilde{\kappa}_{j}^{k+2, l-1}, \\
\Theta_{p} \rightarrow \Theta, \quad z_{q} \rightarrow z_{q-1}, \quad \bar{z}_{q} \rightarrow \bar{z}_{q-1} \quad(p<q \leqslant l),
\end{gathered}
$$

where $\left(\partial / \partial \tau_{p}\right)_{0}$ and $(\partial / \partial \Psi)_{0}$ denote the differentiations at $\tau_{p}=0$ and $\Psi=0$ respectively, and we understand by $\left.\right|_{(4.12)}$ that the replacement or the change of the suffices (4.12) is carried out (this is nothing but the replacement (4.9) restricted to the case where $\tau_{p}=0$ and $\Psi=0$ ).

4.3. Case $\left(2^{+}\right)$. The root $\alpha$ in $\Pi\left(F_{+}\right)$with $F_{+} \subset H_{j}^{k, l}$ corresponding to this case is given by $\alpha(X)=2 t_{j}$. Similarly as for Case (1), let us determine $H_{\alpha}^{\prime}, H_{\beta}^{\prime}, g \in G$ such that $H_{j}^{k, l} \stackrel{\alpha, g}{\longrightarrow} H_{j-1}^{k+1, l}$ and $w \in W_{G}\left(\mathfrak{G}_{c}^{k+1, l}\right)$ such that $P\left(\mathfrak{h}_{\alpha}\right)^{\nu}=$ $P\left(\mathfrak{h}^{k+1, l}\right)^{w}$, where $\mathfrak{h}=\mathfrak{h}^{k, l}$ and $\nu=\operatorname{Ad}(g) \mid \mathfrak{h}_{\alpha}$.

Let us first consider the case $n=1$. Then choosing the root vectors $X_{ \pm \alpha}$ from $\mathfrak{g}_{1} \cong \mathfrak{g} \mathfrak{p}(1, \boldsymbol{R})$ appropriately, we get

$$
H_{\alpha}^{\prime}=\left(\begin{array}{ll}
0 & 1 \\
1 & 0
\end{array}\right), \quad X_{\varepsilon \alpha}^{\prime}=\frac{\sqrt{-1}}{2}\left(\begin{array}{ll}
-\varepsilon & 1 \\
-1 & \varepsilon
\end{array}\right) \quad(\varepsilon= \pm 1) .
$$

Hence

$$
H_{\beta}^{\prime}=\sqrt{-1}\left(X_{\alpha}^{\prime}-X_{-\alpha}^{\prime}\right)=\left(\begin{array}{rr}
1 & 0 \\
0 & -1
\end{array}\right)=\frac{1}{\sqrt{-1}}\left(\begin{array}{cc}
\sqrt{-1} & 0 \\
0 & -\sqrt{-1}
\end{array}\right) .
$$

In the general case of $n$, consider an isomorphism $\phi$ of $\mathfrak{g}_{1}$ into $\mathfrak{g}_{n}$ given as follows. For an element $Y=\left(y_{r s}\right)_{1 \leqslant r, s \leqslant 2}$ in $g_{1}$, we make correspond an element $X=\left(x_{r s}\right)_{1 \leqslant r, s \leqslant 2 n}$ in $\mathfrak{g}_{n}$ such that 


$$
\left(x_{r s}\right)_{r, s=k+2 l+j, n+k+2 l+j}=Y
$$

and all the other components $x_{r s}$ 's are zero. Then we see that the images $\phi\left(H_{\alpha}^{\prime}\right), \phi\left(X_{ \pm \alpha}^{\prime}\right)$ and $\phi\left(\sqrt{-1} H_{\beta}^{\prime}\right)$ of the elements in (4.13) and (4.14) can be taken as the corresponding elements in $\mathfrak{g}_{n}$, and so they are denoted again by $H_{\alpha}^{\prime}, X_{ \pm \alpha}^{\prime}$ and $\sqrt{-1} H_{\beta}^{\prime}$ respectively.

Put $g \in G_{n}$ as

$$
g=\left(\begin{array}{ccccccc}
1_{k} & & & & & & \\
& 0 & 1 & & & & \\
& 1_{2 l+j-1} & 0 & & & & \\
& & & 1_{n-2 l-j} & & & \\
& & & & 0 & 1 & \\
& & & & 1_{2 l+j-1} & 0 & \\
& & & & & & 1_{n-k-2 l-j}
\end{array}\right) .
$$

Then $\operatorname{Ad}(g) \mathfrak{h}_{\alpha}=\mathfrak{h}^{k+1, l}$ with $\mathfrak{h}=\mathfrak{h}^{k, l}$, and $H^{k, l} \stackrel{\alpha, g}{\longrightarrow} H_{j-1}^{k+1, l}$.

We use the co-ordinates (2.9) in $H^{k, l}$ and the analogous ones in $H^{k+1, l}$. "Then we get

$$
\begin{array}{ll}
\partial\left(H_{\alpha}^{\prime}\right)=\frac{\partial}{\partial t_{j}} & \text { on } H^{k, l}, \\
\partial\left(H_{\tilde{\beta}}^{\prime}\right)=\partial\left(\operatorname{Ad}(g) H_{\beta}^{\prime}\right)=\frac{\partial}{\partial \Phi_{k+1}} & \text { on } H^{k+1, l},
\end{array}
$$

where $\tilde{\beta}=\nu \beta$ with $\nu=\operatorname{Ad}(g) \mid \mathfrak{h}_{\alpha}$.

Let us determine $w \in W\left(\mathfrak{h}_{c}^{k+1, l}\right)$ such that $P\left(\mathfrak{h}_{\alpha}\right)^{\nu}=P\left(\mathfrak{h}^{k+1, l}\right)^{w}$. The set of positive roots $P\left(\mathfrak{h}^{k+1, l}\right)$ is defined in $\S 2.2$ as follows: introduce in $\mathfrak{h}^{k+1, l}$ the co-ordinates $\left(x_{1}, x_{2}, \cdots, x_{n}\right)$ as

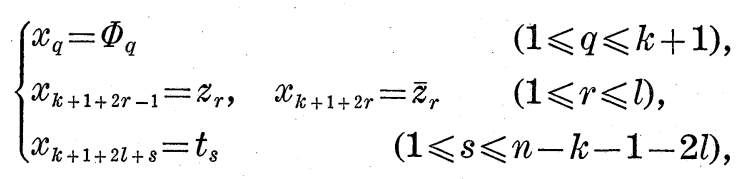

and denote by $X$ the element in $\mathfrak{h}^{k+1, l}$ with these co-ordinates, then

$$
P\left(\mathfrak{G}^{k+1, l}\right)=\left\{\alpha ; \alpha(X)=x_{q} \pm x_{r}(1 \leqslant q<r \leqslant n), 2 x_{q}(1 \leqslant q \leqslant n)\right\} .
$$

On the other hand, we see that $P\left(\mathfrak{h}_{\alpha}\right)^{\nu}$ is given as follows: put

$$
\begin{aligned}
\left(y_{1}, y_{2}, \cdots, y_{n}\right)= & \left(x_{1}, x_{2}, \cdots, x_{k}, \hat{x}_{k+1},\right. \\
& x_{k+2}, x_{k+3}, \cdots, x_{k+2 l+j}, x_{k+1}, x_{k+2 l+j+1}, \\
& \left.x_{k+2 l+j+2}, \cdots, x_{n}\right),
\end{aligned}
$$

then 


$$
P\left(\mathfrak{h}_{\alpha}\right)^{\nu}=\left\{\alpha ; \alpha(X)=y_{q} \pm y_{r}(1 \leqslant q<r \leqslant n), 2 y_{q}(1 \leqslant q \leqslant n)\right\} .
$$

This means that $P\left(\mathfrak{h}_{\alpha}\right)^{v}$ is obtained from $P(\mathfrak{h})$ with $\mathfrak{h}=\mathfrak{h}^{k, l}$ by making the following replacements in the co-ordinates (2.6) in $\mathfrak{h}^{k, l}$ :

$$
\left\{\begin{array}{l}
t_{j} \rightarrow \Phi_{k+1}, \\
t_{q} \rightarrow t_{q-1}
\end{array} \quad(j<q \leqslant m=n-k-2 l) .\right.
$$

Quite analogously as for Case (1), the permutation in the right hand side of (4.18) gives the element $w^{-1} \in W\left(\mathfrak{h}_{c}^{k+1, l}\right)$. Hence

$$
\operatorname{sgn}(w)=(-1)^{j-1} .
$$

The subsets $\Sigma_{\alpha} \cap H_{j}^{k, l}$ and $\Sigma_{\bar{\beta}} \cap H_{j-1}^{k+1, l}$ are given by $t_{j}=0$ and $\Phi_{k+1}=0$ respectively. Therefore we see from (4.16), (4.19) and (4.20) that the equations (1.20) corresponding to $H_{j}^{k, l} \stackrel{\alpha, g}{\longrightarrow} H_{j-1}^{k+1, l}$ are given as follows: for $M=0$, $1,2, \cdots$,

$$
\begin{gathered}
\left.\left(\frac{\partial}{\partial t_{j}}\right)_{0}^{2 M+1} \tilde{\kappa}_{j}^{k, l}\right|_{(4.22)}=(-1)^{j-1}\left(\frac{\partial}{\partial \Phi_{k+1}}\right)_{0}^{2 M+1} \tilde{\kappa}_{j-1}^{k+1, l} \\
t_{q} \rightarrow t_{q-1} \quad(j<q \leqslant m),
\end{gathered}
$$

where $\left.\right|_{(4.22)}$ means that the replacements (4.22) are carried out.

4.4. Case (2-). The root $\alpha$ in $\Pi\left(F_{+}\right)$with $F_{+} \subset H_{j}^{k, l}$ corresponding to this case is given by $\alpha(X)=2 t_{m}$. The calculations are quite analogous as for Case $\left(2^{+}\right)$. The difference is that the subsets $\Sigma_{\alpha} \cap H_{j}^{k, l}$ and $\Sigma_{\tilde{\beta}} \cap H_{j}^{k+1, l}$ are given by the equations $t_{m}=0$ and $\Phi_{k+1}=\pi i(i=\sqrt{-1})$ respectively. Thus we see that the equations (1.20) corresponding to $H_{j}^{k, l} \stackrel{\alpha, g}{\longrightarrow} H_{j}^{k+1, l}$ are given as follows: for $M=0,1,2, \cdots$,

$$
\left(\frac{\partial}{\partial t_{m}}\right)_{0}^{2 M+1} \tilde{\kappa}_{j}^{k, l}=(-1)^{m-1}\left(\frac{\partial}{\partial \Phi_{k+1}}\right)_{\pi i}^{2 M+1} \tilde{\kappa}_{j}^{k+1, l}
$$

where $\left(\partial / \partial \Phi_{k+1}\right)_{\pi i}$ denotes the differentiation at $\Phi_{k+1}=\pi i$, and the replacement. of the co-ordinates corresponding to (4.22) is trivial in this case.

4.5. Case $\left(3^{+}\right)$. The roots in $\Pi\left(F_{+}\right)$with $F_{+} \subset H_{j}^{k, l}$ corresponding to this case are given as $\alpha(X)=t_{p}-t_{p+1}(1 \leqslant p \leqslant j-1)$. Let us calculate $H_{\alpha}^{\prime}$, $H_{\beta}^{\prime}, g \in G$ such that $H_{j}^{k, l} \stackrel{\alpha, g}{\longrightarrow} H_{j j-2}^{k, l+1}$, and $w \in W\left(\mathfrak{h}_{c}^{k, l+1}\right)$ such that $P\left(\mathfrak{h}_{\alpha}\right)^{\nu}=$ $P\left(\mathfrak{h}^{k, l+1}\right)^{w}$, where $\mathfrak{h}=\mathfrak{h}^{k, l}$ and $\nu=\operatorname{Ad}(g) \mid \mathfrak{h}_{\alpha}$.

Let us first consider the case $n=2$. In this case, $\mathfrak{h}^{k, l}=\mathfrak{h}^{0,0}$. Choosing the root vectors $X_{ \pm \alpha}$ from $g_{2} \cong \mathfrak{Z} p(2, R)$ appropriately, we get 
$(4.24)$

$$
H_{\alpha}^{\prime}=\left(\begin{array}{rrrr} 
& & 1 & 0 \\
1 & 0 & 0 & -1 \\
0 & -1 &
\end{array}\right), \quad X_{s \alpha}^{\prime}=\frac{1}{2}\left(\begin{array}{rrrr}
0 & \varepsilon & 0 & 1 \\
-\varepsilon & 0 & 1 & 0 \\
0 & 1 & 0 & \varepsilon \\
1 & 0 & -\varepsilon & 0
\end{array}\right) \quad(\varepsilon= \pm 1)
$$

and hence

$$
H_{\beta}^{\prime}=\sqrt{-1}\left(X_{\alpha}^{\prime}-X_{-\alpha}^{\prime}\right)=\frac{1}{\sqrt{-1}}\left(\begin{array}{rrrr}
0 & -1 & & \\
1 & 0 & & \\
& & 0 & -1 \\
& & 1 & 0
\end{array}\right) \text {. }
$$

Note that $\mathfrak{h}_{\alpha}=R H+R \sqrt{-1} H_{\beta}^{\prime}$, where

$$
H=\left(\begin{array}{ll}
0_{2} & j_{2} \\
j_{2} & 0_{2}
\end{array}\right) \quad \text { with } \quad j_{2}=\left(\begin{array}{ll}
0 & 1 \\
1 & 0
\end{array}\right)
$$

Put $g_{1} \in G_{2}$ as

$$
g_{1}=\left(\begin{array}{ll}
u & 0_{2} \\
0_{2} & u
\end{array}\right), \quad \text { where } \quad u=\frac{1}{\sqrt{2}}\left(\begin{array}{rr}
-\sqrt{-1} & 1 \\
\sqrt{-1} & 1
\end{array}\right) \in U(2) .
$$

Then we get

$$
\left\{\begin{array}{l}
\operatorname{Ad}\left(g_{1}\right)\left(\sqrt{-1} H_{\beta}^{\prime}\right)=\operatorname{diag}(\sqrt{-1},-\sqrt{-1},-\sqrt{-1}, \sqrt{-1}) \\
\operatorname{Ad}\left(g_{1}\right) H=H
\end{array}\right.
$$

Hence $\operatorname{Ad}\left(g_{1}\right) \mathfrak{h}_{\alpha}=\mathfrak{h}^{0,1}$, where $\mathfrak{h}=\mathfrak{h}^{0,0}$.

In the general case of $n$, consider an isomorphism $\phi$ of $g_{2}$ into $\mathfrak{g}_{n}$ given as follows. For an element $Y=\left(y_{r s}\right)_{1 \leqslant r, s \leqslant 4}$ in $g_{2}$, we make correspond an element $X=\left(x_{r s}\right)_{1 \leqslant r, s \leqslant 2 n}$ in $\mathfrak{g}_{n}$ such that

$$
\left(x_{r s}\right)_{r, s=k+2 l+p-1, k+2 l+p, n+k+2 l+p-1, n+k+2 l+p}=Y
$$

and all the other $x_{r s}$ 's are zero. Then we can take $\phi\left(H_{\alpha}^{\prime}\right), \phi\left(X_{ \pm \alpha}^{\prime}\right)$ and $\phi\left(\sqrt{-1} H_{\beta}^{\prime}\right)$ as the corresponding elements for $\mathfrak{g}_{n}$, and so they are denoted again by $H_{\alpha}^{\prime}, X_{ \pm \alpha}^{\prime}$ and $\sqrt{-1} H_{\beta}^{\prime}$ respectively.

Let $u \in U(2)$ be as in (4.26) and put $g \in G_{n}$ as

$$
g=\left(\begin{array}{ccccccc}
\mathbf{1}_{k+2 l} & & & & & & \\
& 0 & u & & & & \\
& \mathbf{1}_{p-1} & 0 & & & & \\
& & & \mathbf{1}_{n-p-1} & & & \\
& & & & 0 & u & \\
& & & & \mathbf{1}_{p-1} & 0 & \\
& & & & & & \mathbf{1}_{m-p-1}
\end{array}\right) \text {, }
$$


where $m=n-k-2 l$. Then we get $\operatorname{Ad}(g) \mathfrak{h}_{\alpha}=\mathfrak{h}^{k, l+1}$ with $\mathfrak{h}=\mathfrak{h}^{k, l}$, and $H_{j}^{k, l}$ $\stackrel{\alpha, g}{\longrightarrow} H_{j-2}^{k, l+1}$.

We use the co-ordinates (2.9) in $H^{k, l}$ and the analogous ones in $H^{k, l+1}$, and put on $H^{k, l}$

$$
t_{p}=\tau+\sigma, \quad t_{p+1}=\tau-\sigma .
$$

Then we get

$$
\begin{array}{ll}
\partial\left(H_{\alpha}^{\prime}\right)=\frac{\partial}{\partial \sigma} & \text { on } H^{k, l}, \\
\partial\left(H_{\tilde{\beta}}^{\prime}\right)=\partial\left(\operatorname{Ad}(g) H_{\beta}^{\prime}\right)=\frac{\partial}{\partial \Theta_{l+1}} & \text { on } H^{k, l+1},
\end{array}
$$

where $\tilde{\beta}=\nu \beta$ with $\nu=\operatorname{Ad}(g) \mid \mathfrak{h}_{\alpha}$.

Let us now determine $w \in W\left(\mathfrak{h}_{c}^{k, l+1}\right)$ such that $P\left(\mathfrak{h}_{\alpha}\right)^{\nu}=P\left(\mathfrak{h}^{k, l+1}\right)^{w}$. The set of positive roots $P\left(\mathfrak{h}^{k, l+1}\right)$ is defined in $\S 2.2$ as follows. Introduce in $\mathfrak{h}^{k, l+1}$ the co-ordinates $\left(x_{1}, x_{2}, \cdots, x_{n}\right)$ as

$$
\begin{cases}x_{q}=\Phi_{q} & (1 \leqslant q \leqslant k), \\ x_{k+2 r-1}=z_{r}, \quad x_{k+2 r}=\bar{z}_{r} & (1 \leqslant r \leqslant l+1), \\ x_{k+2 l+2+s}=t_{s} & (1 \leqslant s \leqslant n-k-2 l-2),\end{cases}
$$

and denote by $X$ the element in $\mathfrak{h}^{k, l+1}$ with these co-ordinates, then

$$
P\left(\mathfrak{h}^{k, l+1}\right)=\left\{\alpha ; \alpha(X)=x_{q} \pm x_{r}(1 \leqslant q<r \leqslant n), 2 x_{q}(1 \leqslant q \leqslant n)\right\} .
$$

On the other hand, we see that $P\left(\mathfrak{h}_{\alpha}\right)^{\text {y }}$ is given as follows: put

$$
\begin{aligned}
\left(y_{1}, y_{2}, \cdots, y_{n}\right)= & \left(x_{1}, x_{2}, \cdots, x_{k+2 l}, \hat{x}_{k+2 l+1},\right. \\
& \hat{x}_{k+2 l+2}, x_{k+2 l+3}, x_{k+2 l+4}, \cdots, x_{k+2 l+p+1}, x_{k+2 l+1}, x_{k+2 l+2}, \\
& \left.x_{k+2 l+p+2}, x_{k+2 l+p+3}, \cdots, x_{n}\right)
\end{aligned}
$$

then

$$
P\left(\mathfrak{h}_{\alpha}\right)^{\nu}=\left\{\alpha ; \alpha(X)=y_{q} \pm y_{r}(1 \leqslant q<r \leqslant n), 2 y_{q}(1 \leqslant q \leqslant n)\right\} .
$$

This means that $P\left(\mathfrak{h}_{\alpha}\right)^{\nu}$ can be obtained from $P(\mathfrak{h})$ with $\mathfrak{h}=\mathfrak{h}^{k, l}$ by making the following replacements in the co-ordinates $(2.6)$ in $\mathfrak{h}^{k, l}$ :

$$
\left\{\begin{array}{l}
t_{p}=\tau+\sigma \rightarrow \tau_{l+1}+\Theta_{l+1}=z_{l+1}, \\
t_{p+1}=\tau-\sigma \rightarrow \tau_{l+1}-\Theta_{l+1}=\bar{z}_{l+1}, \\
t_{q} \rightarrow t_{q-1} \quad(p+1<q \leqslant m=n-k-2 l) .
\end{array}\right.
$$


Quite analogously as for Case (1), the permutation in the right hand side of (4.32) gives the element $w^{-1} \in W\left(\mathfrak{G}_{c}^{k, l+1}\right)$. Hence

$$
\operatorname{sgn}(w)=1
$$

The subsets $\Sigma_{\alpha} \cap H_{j}^{k, l}$ and $\Sigma_{\tilde{\beta}} \cap H_{j-2}^{k, l+1}$ are given by the equations $\sigma=0$ and $\Theta_{l+1}=0$ respectively. Thus we see from (4.30), (4.32) and (4.34) that the equations (1.20) corresponding to $H_{j}^{k, l} \stackrel{\alpha, g}{\longrightarrow} H_{j-2}^{k, l+1}$ are expressed as

$$
\begin{aligned}
&\left.\left(\frac{\partial}{\partial \sigma}\right)_{0}^{2 M+1} \tilde{\kappa}_{j}^{k, l}\right|_{(4.36)}=\left(\frac{\partial}{\partial \Theta_{l+1}}\right)_{0}^{2 M+1} \tilde{\kappa}_{j-2}^{k, l+1} \quad(M=0,1,2, \cdots), \\
&\left\{\begin{array}{l}
\tau \rightarrow \tau_{l+1}, \\
t_{q} \rightarrow t_{q-2}
\end{array} \quad(p+1<q \leqslant m) .\right.
\end{aligned}
$$

4.6. Case (3-). The roots $\alpha$ in $\Pi\left(F_{+}\right)$with $F_{+} \subset H_{j}^{k, l}$ corresponding to this case is given by $\alpha(X)=t_{p}-t_{p+1}(j<p<m)$. The calculations are quite analogous as for Case $\left(3^{+}\right)$and we put

$$
t_{p}=\tau+\sigma, \quad t_{p+1}=\tau-\sigma .
$$

The difference is that the subsets $\Sigma_{\alpha} \cap H_{j}^{k, l}$ and $\Sigma_{\tilde{\beta}} \cap H_{j}^{k, l+1}$ are given by $\sigma=0$ and $\Theta_{l+1}=\pi i(i=\sqrt{-1})$ respectively. Thus we see that the equations (1.20) corresponding to $H_{j}^{k, l} \stackrel{\alpha, g}{\longrightarrow} H_{j}^{k, l+1}$ are given as

$$
\begin{aligned}
\left.\left(\frac{\partial}{\partial \sigma}\right)_{0}^{2 M+1} \tilde{\kappa}_{j}^{k, l+1}\right|_{(4.39)} & =\left(\frac{\partial}{\partial \Theta_{l+1}}\right)_{\pi i}^{2 M+1} \tilde{\kappa}_{j}^{k, l+1} \quad(M=0,1,2, \cdots), \\
& \left\{\begin{array}{l}
\tau \rightarrow \tau_{l+1}, \\
t_{q} \rightarrow t_{q-2}
\end{array} \quad(p+1<q \leqslant m),\right.
\end{aligned}
$$

where $\left(\partial / \partial \Theta_{l+1}\right)_{\pi i}$ denotes the differentiation at $\Theta_{l+1}=i \theta_{l+1}=\pi i$.

\section{The equations on the system of functions $\tilde{\kappa}_{j}^{k, l}$}

5.1. Let us rewrite Theorem 1 for $G=G_{n} \cong S p(n, \boldsymbol{R})$ by means of the functions $\tilde{\kappa}_{j}^{k, l}$. Introduce in $H^{k, l}$ the co-ordinates $\left(\delta_{1}, \delta_{2}, \cdots, \delta_{n}\right)$ as in $\S 2.3$, and consider the analytic function $\tilde{\kappa}_{j}^{k, l}$ on $F_{+} \subset H_{j}^{k, l}$ as a function of these co-ordinates. Recall that

$$
\begin{cases}\delta_{q}=e^{\Phi_{q}}, \quad \Phi_{q}=i \varphi_{q} & (1 \leqslant q \leqslant k) \\ \delta_{k+2 r-1}=e^{z_{r}}, \quad \delta_{k+2 r}=e^{\bar{z}_{r}}, \quad z_{r}=\tau_{r}+\Theta_{r}, \quad \Theta_{r}=i \theta_{r} & (1 \leqslant q \leqslant l) \\ \delta_{k+2 l+s}=\varepsilon_{s} e^{t_{s}} & (1 \leqslant s \leqslant m) .\end{cases}
$$


Let us first consider the condition (i) in Theorem 1. By the result on $W_{G}\left(F_{+}\right)$in $\S 2.5$, it is expressed as follows.

( I ) The analytic function $\tilde{\kappa}_{j}^{k, l}$ on $\boldsymbol{F}_{+} \subset H_{j}^{k, l}$ is

(a) skew-symmetric in $\left(e^{\Phi_{1}}, e^{\Phi_{2}}, \cdots, e^{\Phi_{k}}\right)$;

(b) invariant under any permutation of the pairs $\left(z_{r}, \bar{z}_{r}\right)(1 \leqslant r \leqslant l)$;

(c) odd in every $\Theta_{r}(1 \leqslant r \leqslant l)$.

Next consider the condition (ii) in Theorem 1. Put

$$
\begin{cases}x_{q}=\Phi_{q} & (1 \leqslant q \leqslant k) ; \\ x_{k+2 r-1}=z_{r}, \quad x_{k+2 r}=\bar{z}_{r} & (1 \leqslant r \leqslant l) ; \\ x_{k+2 l+s}=t_{s} & (1 \leqslant s \leqslant m=n-k-2 l),\end{cases}
$$

and moreover put

$$
\begin{aligned}
& \frac{\partial}{\partial \Phi_{q}}=\frac{1}{i} \frac{\partial}{\partial \varphi_{q}}, \quad \frac{\partial}{\partial z_{r}}=\frac{1}{2}\left(\frac{\partial}{\partial \tau_{r}}+\frac{\partial}{\partial \Theta_{r}}\right), \\
& \frac{\partial}{\partial \bar{z}_{r}}=\frac{1}{2}\left(\frac{\partial}{\partial \tau_{r}}-\frac{\partial}{\partial \Theta_{r}}\right), \quad \frac{\partial}{\partial \Theta_{r}}=\frac{1}{i} \frac{\partial}{\partial \theta_{r}} .
\end{aligned}
$$

Then the subalgebra $I\left(\mathfrak{h}_{c}^{k, l}\right)$ of $S\left(\mathfrak{h}_{c}^{k, l}\right)$, considered as an algebra of differential operators on $H^{k, l}$, consists of the elements of the following form:

$$
P\left(\frac{\partial}{\partial x_{1}}, \frac{\partial}{\partial x_{2}}, \cdots, \frac{\partial}{\partial x_{n}}\right),
$$

where $P\left(Z_{1}, Z_{2}, \cdots, Z_{n}\right)$ are symmetric polynomials of $Z_{1}^{2}, Z_{2}^{2}, \cdots, Z_{n}{ }^{2}$. Therefore the condition (ii) in Theorem 1 is written as follows.

(II) There exist complex numbers $\lambda_{1}, \lambda_{2}, \cdots, \lambda_{n}$ such that every function $\tilde{\kappa}_{j}^{k, l}$ satisfies

$$
P\left(\frac{\partial}{\partial x_{1}}, \frac{\partial}{\partial x_{2}}, \cdots, \frac{\partial}{\partial x_{n}}\right) \tilde{\kappa}_{j}^{k, l}=P\left(\lambda_{1}, \lambda_{2}, \cdots, \lambda_{n}\right) \tilde{\kappa}_{j}^{k, l},
$$

for any symmetric polynomial $P\left(Z_{1}, Z_{2}, \cdots, Z_{n}\right)$ of $Z_{1}^{2}, Z_{2}^{2}, \cdots, Z_{n}^{2}$.

Let us now consider the condition (iii) in Theorem 1. This is studied in the preceeding section and we get the following result.

(III) The functions $\tilde{\kappa}_{j}^{k, l}$ 's satisfy the equations (4.11), (4.12) in Case (1); (4.21), (4.22) in Case $\left(2^{+}\right)$; (4.23) in Case $\left(2^{-}\right) ;(4.35),(4.36)$ in Case $\left(3^{+}\right)$; and (4.38), (4.39) in Case (3-). 
Recall that $\left\{H_{j}^{k, l} ; k+2 l \leqslant n, k \geqslant 0, l \geqslant 0,0 \leqslant j \leqslant m=n-k-2 l\right\}$ is a complete system of representatives of $\operatorname{Car}^{0}(G)$. Then we get from Theorem 1 and the condition (T) the following theorem on $G=G_{n} \cong S p(n, R)$.

THEOREM 4. Assume that for every $H_{j}^{k, l}$, an analytic function $\tilde{\kappa}_{j}^{k, l}$ on $F_{+} \subset H^{k, l}$ is given. Then the system of functions $\left\{\tilde{\kappa}_{j}^{k, l}\right\}$ defines canonically a tempered invariant eigendistribution on $G$ if it satisfies the condition $(\mathrm{T})$ in $\S 1.5$ and the conditions (I), (II) and (III) above.

5.2. In practice the differential equations in the condition (III) are treated in the analogous fashion as was done in [4(d), §2]. Let us explain this for the equations (4.11), (4.12) in Case (1).

Consider $\tilde{\kappa}_{j}^{k, l}$ as a function of the co-ordinates in (5.1) or rather of those in (5.2). Since $\tilde{\kappa}_{j}^{k, l}$ is analytic and satisfies (5.4), we can express it in the form of Taylor series in $\tau_{p}$ arround $\tau_{p}=0$. Replace in this series the variable $\tau_{p}$ by the one $\Psi=i \psi$, then we get an analytic function in $\Psi$, denoted by $\boldsymbol{R}\left(\Psi, \tau_{p}\right) \tilde{\kappa}_{j}^{k, l}$. Then the differential equations (4.11), (4.12) take the following form :

$$
\begin{gathered}
\left.\boldsymbol{R}\left(\Psi, \tau_{p}\right) \tilde{\kappa}_{j}^{k, l}\right|_{(5.6)} \equiv \tilde{\kappa}_{j}^{k+2, l-1} \text { (modulo even functions in } \Psi \text { ), } \\
\Theta_{p} \rightarrow \Theta ; z_{q} \rightarrow z_{q-1}, \quad \bar{z}_{q} \rightarrow \bar{z}_{q-1} \quad(p<q \leqslant l),
\end{gathered}
$$

where $\left.\right|_{(5.6)}$ means that the changes of the suffices of the variables in (5.6) are carried out.

The differential equations in other cases can also be rewritten analogously (for the details, see $\S 8$ below).

5.3. Now let $\pi$ be the character of a representation of $G$ in the discrete series. Then, by the result referred in $\S 1.5$, the function $\tilde{\kappa}^{n, 0}$ on the compact Cartan subgroup $H^{n, 0}$ corresponding to $\pi$ by $\tilde{\kappa}^{n, 0}=\Delta^{n, 0} \pi$ is given as follows.

Let $l_{1}, l_{2}, \cdots, l_{n}$ be positive integers such that

$$
l_{1}>l_{2}>\ldots>l_{n}>0 \text {, }
$$

and let $\nu_{1}, \nu_{2}, \cdots, \nu_{n}$ be 1 or -1 . Put

$$
\Lambda=\left(\lambda_{1}, \lambda_{2}, \cdots, \lambda_{n}\right)=\left(\nu_{1} l_{1}, \nu_{2} l_{2}, \cdots, \nu_{n} l_{n}\right) .
$$

Then $\Lambda$ defines a linear form on $\mathfrak{h}_{e}^{n, 0}$ in such a way that

$$
\Lambda(X)=\lambda_{1} x_{1}+\lambda_{2} x_{2}+\cdots+\lambda_{n} x_{n},
$$

in the co-ordinates $x_{s}=\Phi_{s}(1 \leqslant s \leqslant n)$ of $X$ in $(5.2)$ for $(k, l)=(n, 0)$. Any 
regular character of $H^{n, 0}$ is given by $H^{n, 0} \ni \exp X \rightarrow e^{\Lambda(w X)}$ for some such $A$ and $w \in W_{G}\left(\mathfrak{h}^{n, 0}\right) \cong \mathfrak{S}_{n}$.

On the other hand, since $2^{-1}(\operatorname{dim} G-\operatorname{dim} K)=n(n+1) / 2$, we see from (1.24) that $\pi$ is given as $\pi=\pi_{A}=(-1)^{n(n+1) / 2} \varepsilon(\Lambda) \Theta_{\Lambda}$ for some $\Lambda$. Note that

$$
\varepsilon(A)=\nu_{1} \nu_{2} \cdots \nu_{n},
$$

then we see from (1.22) that the function $\tilde{\kappa}^{n, 0}$ for $\pi=\pi_{A}$ is given as

$$
\tilde{\kappa}^{n, 0}(h)=(-1)^{n(n+1) / 2}\left|\nu_{1} \delta^{\nu_{1} l_{1}}, \nu_{2} \delta^{\nu_{2} l_{2}}, \cdots, \nu_{n} \delta^{\nu_{n} l_{n}}\right|_{\delta=\delta_{1}, \delta_{2}}, \cdots, \delta_{n},
$$

where $\delta_{s}=e^{\Phi_{s}}(1 \leqslant s \leqslant n)$ are the co-ordinates of $h \in H^{n, 0}$, and the right hand side denotes, except the multiplicative factor $(-1)^{n(n+1) / 2}$, the determinant of order $n$ whose $s$-th row is equal to $\left(\nu_{1} \delta_{s}^{\nu_{1} l_{1}}, \nu_{2} \delta_{s}^{\nu_{2} l_{2}}, \cdots, \nu_{n} \delta_{s}^{\nu_{n} l_{n}}\right)$.

In the following we shall determine the functions $\tilde{\kappa}_{j}^{k, l}$ corresponding to the character $\pi_{\Lambda}$.

\section{§ 6. Explicit formulas for $S p(2, \boldsymbol{R})$ and $S p(3, \boldsymbol{R})$}

In this section we give without proofs the explicit formulas of the characters of discrete series representations for the groups $G_{2} \cong S p(2, R)$ and $G_{3} \cong S p(3, R)$. They help us to understand well the general formulas for $G_{n}$. For the groups $G=G_{2}$ and $G_{3}$, we calculated the functions $\tilde{\kappa}_{j}^{k, l}$ 's one by one starting from $\tilde{\kappa}^{n, 0}$ and according to the order $\prec$ in $\operatorname{Car}^{0}(G)$ by applying Theorem 4.

6.1. Let us introduce some notations which will be employed frequently in the following.

Let $l_{1}, l_{2}, \cdots, l_{k}$ be positive integers such that $l_{1}>l_{2}>\ldots>l_{k}>0$ and $\nu_{s}=$ \pm 1 for $1 \leqslant s \leqslant k$. Let $\delta_{1}, \delta_{2}, \cdots, \delta_{k}$ be indeterminates and put

$$
\begin{aligned}
& \xi_{k}\left(\delta_{1}, \delta_{2}, \cdots, \delta_{k} ; \nu_{1} l_{1}, \nu_{2} l_{2}, \cdots, \nu_{k} l_{k}\right) \\
& =\operatorname{det}\left(\nu_{i} \delta_{j}^{\nu_{i} l} l_{i}\right)_{1 \leqslant i, j \leqslant k} \\
& =\left|\nu_{1} \delta^{\nu_{1} l_{1}}, \nu_{2} \delta^{\nu_{2} l_{2}}, \cdots, \nu_{k} \delta^{\nu_{k} l_{k}}\right|_{\delta_{\delta=\delta_{1}} \delta_{2}, \cdots, \delta_{n}} .
\end{aligned}
$$

For $l_{1}>l_{2}>0$ and $\nu_{1}, \nu_{2}= \pm 1$, we put

$$
\begin{gathered}
\eta_{2}\left(\delta_{1}, \delta_{2} ; \nu_{1} l_{1}, \nu_{2} l_{2}\right)=\left|\begin{array}{cc}
-\delta_{1}^{-l_{1}} & -\nu_{1} \nu_{2} \delta_{1}^{-\nu_{1} \nu_{2} l_{2}} \\
-\delta_{2}^{-l_{1}} & -\nu_{1} \nu_{2} \delta_{2}^{-\nu_{1} \nu_{2} l_{2}}
\end{array}\right| ; \\
\zeta_{2}\left(\delta_{1}, \delta_{2} ; \nu_{1} l_{1}, \nu_{2} l_{2}\right)=\left|\begin{array}{cc}
-\delta_{1}^{-l_{1}} & -\delta_{1}^{-l_{2}} \\
-\delta_{2}^{-l_{1}} & -\nu_{1} \nu_{2}\left(\delta_{2}^{-l_{2}}+\delta_{2}^{l_{2}}\right)+\delta_{2}^{l_{2}}
\end{array}\right| ; \\
\chi\left(\delta_{1}, \delta_{2} ; \nu_{1} l_{1}, \nu_{2} l_{2}\right)=\left|\begin{array}{ll}
-\delta_{1}^{-l_{1}} & -\delta_{2}^{-l_{2}} \\
-\delta_{2}^{-l_{1}} & -\delta_{2}^{-l_{2}}
\end{array}\right|
\end{gathered}
$$




$$
\chi\left(\delta_{1} ; \nu_{1} l_{1}\right)=-\delta_{1}^{-l_{1}}
$$

Then

$$
\begin{aligned}
\zeta_{2}\left(\delta_{1}, \delta_{2} ; \nu_{1} l_{1}, \nu_{2} l_{2}\right)= & \chi\left(\delta_{1}, \delta_{2} ; \nu_{1} l_{1}, \nu_{2} l_{2}\right) \\
& -\left(\nu_{1} \nu_{2}-1\right) \delta_{1}^{-l_{1}}\left(\delta_{2}^{-l_{2}}+\delta_{2}^{l_{2}}\right) .
\end{aligned}
$$

6.2. The group $G_{2} \cong S p(2, R)$. A complete system of representatives of $\operatorname{Car}^{0}(G)$ is given by

$$
\left\{H^{2,0} ; H^{0,1} ; H_{1}^{1,0}, H_{0}^{1,0} ; H_{2}^{0,0}, H_{1}^{0,0}, H_{0}^{0,0}\right\},
$$

where $H^{k, l}$ denotes $H_{0}^{k, l}$ if $H^{k, l}$ is connected or $k+2 l=n$. The order in $\operatorname{Car}^{0}(G)$ is illustrated in Figure 3.2.

As in $\S 2.3$, let $\left(\delta_{1}, \delta_{2}\right)$ be respectively

$$
\begin{cases}\left(e^{\Phi_{1}}, e^{\Phi_{2}}\right) \text { for } H^{2,0} ; & \left(e^{z_{1}}, e^{\bar{z}_{1}}\right) \text { for } H^{0,1} ; \\ \left(e^{\Phi_{1}}, \varepsilon_{1} e^{t_{1}}\right) \text { for } H^{1,0} ; & \left(\varepsilon_{1} e^{t_{1}}, \varepsilon_{2} e^{t_{2}}\right) \text { for } H^{0,0} .\end{cases}
$$

Note that the subset $F_{+}$of $H_{j}^{k, l}$ is given by (2.12) and that $(-1)^{n(n+1) / 2}=-1$ for $n=2$. Put $A=\left(\lambda_{1}, \lambda_{2}\right)=\left(\nu_{1} l_{1}, \nu_{2} l_{2}\right)$. Then, for the character $\pi_{\Lambda}$, the functions $\tilde{\kappa}_{j}^{k, l}$ on $F_{+} \subset H_{j}^{k, l}$ are given as follows: let $\left(\delta_{1}, \delta_{2}\right)$ be the co-ordinates of $h \in F_{+} \subset H_{j}^{k, l}$ given in (6.6), then

$$
\begin{array}{rlr}
\tilde{\kappa}^{2,0}(h) & =-\xi_{2}\left(\delta_{1}, \delta_{2} ; \lambda_{1}, \lambda_{2}\right) ; \\
\tilde{\kappa}^{0,1}(h) & =-\eta_{2}\left(\delta_{1}, \delta_{2} ; \lambda_{1}, \lambda_{2}\right) ; & \\
\tilde{\kappa}_{j}^{1,0}(h) & =-\left\{-\xi_{1}\left(\delta_{1} ; \lambda_{1}\right) \cdot \chi\left(\delta_{2} ; \lambda_{2}\right)+\xi_{1}\left(\delta_{1} ; \lambda_{2}\right) \cdot \chi\left(\delta_{2} ; \lambda_{1}\right)\right\} \\
& =-\left|\begin{array}{ll}
\nu_{1} \delta^{\nu_{1} l_{1}} & \nu_{2} \delta^{\nu_{2} l_{2}} \\
-\delta_{2}^{-l_{1}} & -\delta_{2}^{-l_{2}}
\end{array}\right| & (j=1,0) ; \\
\tilde{\kappa}_{j}^{0,0}(h) & =-\zeta_{2}\left(\delta_{1}, \delta_{2} ; \lambda_{1}, \lambda_{2}\right) & (j=2,0) ; \\
\tilde{\kappa}_{1}^{0,0}(h) & =-\chi\left(\delta_{1}, \delta_{2} ; \lambda_{1}, \lambda_{2}\right) . &
\end{array}
$$

Note that $\zeta_{2}$ and so $\tilde{\kappa}_{j}^{0,0}$ for $j=2,0$ take apparently different forms according as $\nu_{1} \nu_{2}=1$ or -1 . The cases where $\nu_{1} \nu_{2}=1$ correspond to the holomorphic discrete series and their contragradient ones (the latters are holomorphic with respect to another complex structure on $G / K)$.

6.3. The group $G_{3} \cong S p(3, \boldsymbol{R})$. A complete system of representatives of $\operatorname{Car}^{0}(G)$ is given by

$$
\left\{H^{3,0} ; H^{1,1} ; H_{1}^{2,0}, H_{0}^{2,0} ; H_{1}^{0,1}, H_{0}^{0,1} ; H_{2}^{1,0}, H_{1}^{1,0}, H_{0}^{1,0} ; H_{3}^{0,0}, H_{2}^{0,0}, H_{1}^{0,0}, H_{0}^{0,0}\right\} .
$$

The order $\prec$ in $\operatorname{Car}^{0}(G)$ is illustrated in Figure 3.2.

As in $\S 2.3$, let $\left(\delta_{1}, \delta_{2}, \delta_{3}\right)$ be respectively 


$$
\begin{array}{ll}
\left(e^{\Phi_{1}}, e^{\Phi_{2}}, e^{\Phi_{3}}\right) \text { for } H^{3,0} ; & \left(e^{\Phi_{1}}, e^{z_{1}}, e^{\Sigma_{1}}\right) \text { for } H^{1,1} ; \\
\left(e^{\Phi_{1}}, e^{\Phi_{2}}, \varepsilon_{1} e^{t_{1}}\right) \text { for } H^{2,0} ; & \left(e^{z_{1}}, e^{z_{1}}, \varepsilon_{1} e^{t_{1}}\right) \text { for } H^{0,1} ; \\
\left(e^{\Phi_{1}}, \varepsilon_{1} e^{t_{1}}, \varepsilon_{2} e^{t_{2}}\right) \text { for } H^{1,0} ; & \left(\varepsilon_{1} e^{t_{1}}, \varepsilon_{2} e^{t_{2}}, \varepsilon_{3} e^{t_{3}}\right) \text { for } H^{0,0}
\end{array}
$$

Note that $(-1)^{n(n+1) / 2}=1$ for $n=3$. Put $\Lambda=\left(\lambda_{1}, \lambda_{2}, \lambda_{3}\right)=\left(\nu_{1} l_{1}, \nu_{2} l_{2}, \nu_{3} l_{3}\right)$. By the method mentioned above, we get the system of functions $\tilde{\kappa}_{j}^{k, l}$ for $h \in F_{+}$ $\subset H_{j}^{k, l}$ corresponding to the character $\pi_{A}$. For some $(k, l, j)$, the resulting explicit forms of $\tilde{\kappa}_{j}^{k, l}$ have very different appearances depending on $\left(\nu_{1}, \nu_{2}, \nu_{3}\right)$ (but not on $\left(l_{1}, l_{2}, l_{3}\right)$ ).

Let $\left(\delta_{1}, \delta_{2}, \delta_{3}\right)$ be the co-ordinates of $h \in F_{+} \subset H_{j}^{k, l}$, then

$$
\begin{aligned}
\tilde{\kappa}^{3,0}(h)= & \xi_{3}\left(\delta_{1}, \delta_{2}, \delta_{3} ; \lambda_{1}, \lambda_{2}, \lambda_{3}\right) ; \\
\tilde{\kappa}^{1,1}(h)= & \xi_{1}\left(\delta_{1} ; \lambda_{1}\right) \cdot \eta_{2}\left(\delta_{2}, \delta_{3} ; \lambda_{2}, \lambda_{3}\right) \\
& -\xi_{1}\left(\delta_{1} ; \lambda_{2}\right) \cdot \eta_{2}\left(\delta_{2}, \delta_{3} ; \lambda_{1}, \lambda_{3}\right)+\xi_{1}\left(\delta_{1} ; \lambda_{3}\right) \cdot \eta_{2}\left(\delta_{2}, \delta_{3} ; \lambda_{1}, \lambda_{2}\right) ; \\
\tilde{\kappa}_{j}^{2,0}(h)= & \xi_{2}\left(\delta_{1}, \delta_{2} ; \lambda_{1}, \lambda_{2}\right) \cdot \chi\left(\delta_{3} ; \lambda_{3}\right) \\
& -\xi_{2}\left(\delta_{1}, \delta_{2} ; \lambda_{1}, \lambda_{3}\right) \cdot \chi\left(\delta_{3} ; \lambda_{2}\right)+\xi_{2}\left(\delta_{1}, \delta_{2} ; \lambda_{2}, \lambda_{3}\right) \cdot \chi\left(\delta_{3} ; \lambda_{1}\right) \\
= & \left|\begin{array}{lll}
\nu_{1} \delta^{\nu_{1} l_{1}} & \nu_{2} \delta^{\nu_{2} l_{2}} & \nu_{3} \delta^{\nu_{3} l_{3}} \\
-\delta_{3}^{-l_{1}} & -\delta_{3}^{-l_{2}} & -\delta_{3}^{-l_{3}}
\end{array}\right|_{\delta=\delta_{1}, \delta_{2}} \quad(j=2,0),
\end{aligned}
$$

where the last term denotes the determinant of order 3 with $\left(\nu_{1} \delta_{i}^{\nu_{1} l_{1}}, \nu_{2} \delta_{i}^{\nu_{2} l_{2}}\right.$, $\left.\nu_{3} \delta_{i}^{\nu_{3} l_{3}}\right)$ as its $i$-th row for $i=1,2$;

$$
\begin{aligned}
& \tilde{\kappa}_{j}^{0,1}(h)=\eta_{2}\left(\delta_{1}, \delta_{2} ; \lambda_{1}, \lambda_{2}\right) \cdot \chi\left(\delta_{3} ; \lambda_{3}\right) \\
& -\eta_{2}\left(\delta_{1}, \delta_{2} ; \lambda_{1}, \lambda_{3}\right) \cdot \chi\left(\delta_{3} ; \lambda_{2}\right)+\eta_{2}\left(\delta_{1}, \delta_{2} ; \lambda_{2}, \lambda_{3}\right) \cdot \chi\left(\delta_{3} ; \lambda_{1}\right) \quad(j=1,0) ; \\
& \tilde{\kappa}_{j}^{1,0}(h)=\xi_{1}\left(\delta_{1} ; \lambda_{1}\right) \cdot \zeta_{2}\left(\delta_{2}, \delta_{3} ; \lambda_{2}, \lambda_{3}\right) \\
& -\xi_{1}\left(\delta_{1} ; \lambda_{2}\right) \cdot \zeta_{2}\left(\delta_{2}, \delta_{3} ; \lambda_{1}, \lambda_{3}\right)+\xi_{1}\left(\delta_{1} ; \lambda_{3}\right) \cdot \zeta_{2}\left(\delta_{2}, \delta_{3} ; \lambda_{1}, \lambda_{2}\right) \quad(j=2,0) ; \\
& \tilde{\kappa}_{1}^{1,0}(h)=\xi_{1}\left(\delta_{1} ; \lambda_{1}\right) \cdot \chi\left(\delta_{2}, \delta_{3} ; \lambda_{2}, \lambda_{3}\right) \\
& -\xi_{1}\left(\delta_{1} ; \lambda_{2}\right) \cdot \chi\left(\delta_{2}, \delta_{3} ; \lambda_{1}, \lambda_{3}\right)+\xi_{1}\left(\delta_{1} ; \lambda_{3}\right) \cdot \chi\left(\delta_{2}, \delta_{3} ; \lambda_{1}, \lambda_{2}\right) \\
& =\left|\begin{array}{lll}
\nu_{1} \delta_{1}^{\nu_{1} l_{1}} & \nu_{2} \delta_{1}^{\nu_{2} l_{2}} & \nu_{3} \delta_{1}^{\nu_{3} l_{3}} \\
-\delta^{-l_{1}} & -\delta^{-l_{2}} & -\delta^{-l_{3}}
\end{array}\right|_{\hat{\delta}=\delta_{2}, \delta_{3}} ; \\
& \tilde{\kappa}_{2}^{0,0}(h)=\zeta_{2}\left(\delta_{1}, \delta_{2} ; \lambda_{1}, \lambda_{2}\right) \cdot \chi\left(\delta_{3} ; \lambda_{3}\right) \\
& -\zeta_{2}\left(\delta_{1}, \delta_{2} ; \lambda_{1}, \lambda_{3}\right) \cdot \chi\left(\delta_{3} ; \lambda_{2}\right)+\zeta_{2}\left(\delta_{1}, \delta_{2} ; \lambda_{2}, \lambda_{3}\right) \cdot \chi\left(\delta_{3} ; \lambda_{1}\right) ; \\
& \tilde{\kappa}_{1}^{0,0}(h)=\chi\left(\delta_{1} ; \lambda_{1}\right) \cdot \zeta_{2}\left(\delta_{2}, \delta_{3} ; \lambda_{2}, \lambda_{3}\right) \\
& -\chi\left(\delta_{1} ; \lambda_{2}\right) \cdot \zeta_{2}\left(\delta_{2}, \delta_{3} ; \lambda_{1}, \lambda_{3}\right)+\chi\left(\delta_{1} ; \lambda_{3}\right) \cdot \zeta_{2}\left(\delta_{2}, \delta_{3} ; \lambda_{1}, \lambda_{2}\right) ; \\
& \tilde{\kappa}_{j}^{0,0}(h)=\left|-\delta^{-l_{1}}-\delta^{-l_{2}}-\delta^{-l_{3}}\right|_{\delta=\delta_{1}, \delta_{2}, \delta_{3}}+S \quad(j=3,0),
\end{aligned}
$$

where

$$
S=-\left(\nu_{1} \nu_{2}-1\right) \delta_{1}^{-l_{1}}\left(\delta_{2}^{-l_{2}}+\delta_{2}^{l_{2}}\right) \delta_{3}^{-l_{3}}+\left(\nu_{1} \nu_{3}-1\right) \delta_{1}^{-l_{1}}\left(\delta_{2}^{-l_{3}}+\delta_{2}^{l_{3}}\right) \delta_{3}^{-l_{2}}
$$




$$
\begin{aligned}
& -\left(\nu_{2} \nu_{3}-1\right) \delta_{1}^{-l_{2}}\left(\delta_{2}^{-l_{3}}+\delta_{2}^{l_{3}}\right) \delta_{3}^{-l_{1}}-\left(\nu_{1} \nu_{2}-1\right)\left(\delta_{1}^{-l_{1}} \delta_{2}^{-l_{3}}+\delta_{1}^{-l_{3}} \delta_{2}^{-l_{1}}\right)\left(\delta_{3}^{-l_{2}}+\delta_{3}^{l_{2}}\right) \\
& +\left\{\left(\nu_{1} \nu_{3}-1\right)-\left(\nu_{2} \nu_{3}-1\right)\right\}\left(\delta_{1}^{-l_{1}} \delta_{2}^{-l_{2}}+\delta_{1}^{-l_{2}} \delta_{2}^{-l_{1}}\right)\left(\delta_{3}^{-l_{3}}+\delta_{3}^{l_{3}}\right) .
\end{aligned}
$$

Note that when $\nu_{1}=\nu_{2}=\nu_{3}= \pm 1$, we get $S=0$ for $\tilde{\kappa}_{j}^{0,0}(j=3,0)$ and moreover every $\tilde{\kappa}_{j}^{k, l}$ has a simple expression. In particular, when $\nu_{1}=\nu_{2}=\nu_{3}=1$, every $\tilde{\kappa}_{j}^{k, l}$ can be expressed by a single formula as

$$
\tilde{\kappa}_{j}^{k, l}(h)=\left|-\delta^{-l_{1}} \quad-\delta^{-l_{2}} \quad-\delta^{-l_{3}}\right|_{\delta=\delta_{1}, \tilde{\delta}_{2}, \hat{\delta}_{3}} .
$$

These cases correspond to the holomorphic discrete series and their contragradient ones (cf. $\S 9$ below).

6.4. To generalize these results for $G=G_{2}$ and $G_{3}$ to the case $G=G_{n}$, it seems natural to use the functions $\xi_{k}, \eta_{2}, \zeta_{2}$ and $\chi$ as fundamental elements. In fact, we shall get in the sequel certain unified expressions of $\tilde{\kappa}_{j}^{k, l}$ 's by means of these functions, valid for all $\Lambda=\left(\lambda_{1}, \lambda_{2}, \cdots, \lambda_{n}\right)=\left(\nu_{1} l_{1}, \nu_{2} l_{2}, \cdots, \nu_{n} l_{n}\right)$.

In this direction, the above expression of the functions $\tilde{\kappa}_{j}^{0,0}(j=3,0)$ for $G_{3}$ are rewritten as follows: denote by $(p, q, r)$ and $(u, v, w)$ permutations of $(1,2,3)$ satisfying respectively

$$
p<q \text { and } u<v
$$

then for $j=3,0$,

$$
\tilde{\kappa}_{j}^{0,0}(h)=\sum_{(p, q, r)} \operatorname{sgn}(p, q, r) \sum_{(u, v, w)} \zeta_{2}\left(\delta_{u}, \delta_{v} ; \lambda_{p}, \lambda_{q}\right) \cdot \chi\left(\delta_{w} ; \lambda_{r}\right),
$$

where the sums runs over all permutations satisfying (6.8).

\section{§7. Explicit formulas for $S p(n, \boldsymbol{R})$}

7.1. In this section we give the explicit formulas of the characters of discrete series representations for the groups $G=G_{n} \cong S p(n, \boldsymbol{R})$. The proof of these formulas will be given in the next section.

A complete system of representatives of $\operatorname{Car}^{0}(G)$ is given by

$$
\left\{H_{j}^{k, l} ; k+2 l \leqslant n, k \geqslant 0, l \geqslant 0,0 \leqslant j \leqslant m=n-k-2 l\right\},
$$

where $H_{j}^{k, l}$ is the connected component of $H^{k, l}$ defined in $\S 2.5$. The order $\prec$ in $\operatorname{Car}^{0}(G)$ is determined by the relations $(1),\left(2^{+}\right),\left(2^{-}\right),\left(3^{+}\right)$and $\left(3^{-}\right)$in Lemma 3.2. As in $\S 5$, we introduce in every Cartan subgroup $H^{k, l}$ the coordinates $\left(\delta_{1}, \delta_{2}, \cdots, \delta_{n}\right)$ by (5.1), and denote in general by $h$ the element of $H^{k, l}$ with the co-ordinates $\left(\delta_{1}, \delta_{2}, \cdots, \delta_{n}\right)$. The open subset $F_{+}$of $H_{j}^{k, l}$ is defined by (2.12). We give the formulas of the bounded analytic functions $\tilde{\kappa}_{j}^{k, l}$ on $F_{+} \subset H_{j}^{k, l}$ for all $H_{j}^{k, l}$. Let $\xi_{k}, \eta_{2}, \zeta_{2}$ and $\chi$ be as in $\S 6.1$.

Let $l_{1}>l_{2}>\ldots>l_{n}>0$ and $\nu_{s}= \pm 1(1 \leqslant s \leqslant n)$ be as in $\S 5.3$, and put 


$$
\Lambda=\left(\lambda_{1}, \lambda_{2}, \cdots, \lambda_{n}\right)=\left(\nu_{1} l_{1}, \nu_{2} l_{2}, \cdots, \nu_{n} l_{n}\right) .
$$

Then, as is seen in $\S 5.3$, the function $\tilde{\kappa}^{n, 0}$ corresponding to the character $\pi_{\Lambda}=(-1)^{n(n+1) / 2} \varepsilon(\Lambda) \Theta_{\Lambda}$ is given by

$$
\tilde{\kappa}^{n, 0}(h)=\xi_{n}\left(\delta_{1}, \delta_{2}, \cdots, \delta_{n} ; \lambda_{1}, \lambda_{2}, \cdots, \lambda_{n}\right) .
$$

7.2. To give the formulas of $\tilde{\kappa}_{j}^{k, l}$ in general, let us introduce some notations. Put $\Omega_{n}=\{1,2, \cdots, n\}$. Fix $(k, l, j)$ and put

$$
J=[j / 2], \quad I=[(m-j) / 2] .
$$

Let $A, B, C, P$ and $Q$ be ordered sets of different integers in $\Omega_{n}$ satisfying the following conditions :

$$
\begin{gathered}
A=\left(a_{1}, a_{2}, \cdots, a_{k}\right), \quad a_{1}<a_{2}<\cdots<a_{k} ; \\
\left\{\begin{array}{l}
B=\left(b_{1}, b_{2}, \cdots, b_{l}\right), \\
C=\left(c_{1}, c_{2}, \cdots, c_{l}\right), \quad b_{i}<c_{i} \quad(1 \leqslant i \leqslant l) ;
\end{array}\right. \\
\left\{\begin{array}{l}
P=\left(p_{1}, p_{2}, \cdots, p_{J+I}\right), \\
Q=\left(q_{1}, q_{2}, \cdots, q_{J+I}\right), \quad p_{i}<q_{i} \quad(1 \leqslant i \leqslant J+I) .
\end{array}\right.
\end{gathered}
$$

We prepare also two symbols $r$ and $s$. The symbol $r$ denotes an integer when $j=2 J+1$, and the empty set $\varnothing$ when $j=2 J$ respectively. The symbol $s$ denotes an integer when $m-j=2 I+1$, and $\varnothing$ when $m-j=2 I$ respectively. This is a practical convention to give simple and unified formulas of $\tilde{\kappa}_{j}^{k, l}$. When both $r$ and $s$ denote integers, we assume that

$$
r<s .
$$

Denote by $\bar{A}$ the set of integers $\left\{a_{1}, a_{2}, \cdots, a_{k}\right\}$, then we assume further that

$$
\bar{A} \cup \bar{B} \cup \bar{C} \cup \bar{P} \cup \bar{Q} \cup\{r, s\}=\Omega_{n} .
$$

Thus the above equality gives a partition of $\Omega_{n}$. For brevity, we denote the collection $(A, B, C, Q, P, r, s)$ simply by $\mathfrak{A}$.

In the above convention, we understand that $\chi\left(\delta_{r}, \delta_{s} ; \lambda_{r}, \lambda_{s}\right)$ denotes $\chi\left(\delta_{r} ; \lambda_{r}\right)$ when $s$ denotes $\varnothing$, and it denotes 1 when both $r$ and $s$ denote $\varnothing$. As will be seen later, this convention causes no ambiguity.

Denote by $B \cdot C$ the following ordered set of integers corresponding to $B$ and $C$ :

$$
B \cdot C=\left(b_{1}, c_{1}, b_{2}, c_{2}, \cdots, b_{l}, c_{l}\right) .
$$

Moreover we denote by 


$$
\operatorname{sgn}(A, B \cdot C, P \cdot Q, r, s)=\operatorname{sgn}(\mathfrak{U})
$$

the sign of the permutation of $\Omega_{n}$ given by arranging $A, B \cdot C, P \cdot Q, r$ and $s$ in this order.

We also prepare two ordered sets $U, V$ of different integers satisfying the following conditions:

$$
\begin{aligned}
& \left\{\begin{array}{l}
U=\left(u_{1}, u_{2}, \cdots, u_{J+I}\right) \\
V=\left(v_{1}, v_{2}, \cdots, v_{J+I}\right), \quad u_{i}<v_{i} \quad(1 \leqslant i \leqslant J+I) ;
\end{array}\right. \\
& v_{1}<v_{2}<\cdots<v_{J}, \quad v_{J+1}<v_{J+2}<\cdots<v_{J+I} .
\end{aligned}
$$

We prepare two symbols $w$ and $x$. The former denotes an integer when $j=2 J+1$ and $\varnothing$ when $j=2 J$ respectively, and the latter denotes an integer when $m-j=2 I+1$ and $\varnothing$ when $m-j=2 I$ respectively. We assume the following condition:

$$
\begin{aligned}
& \left\{u_{i}, v_{i}(1 \leqslant i \leqslant J), w\right\}=\{k+2 l+1, k+2 l+2, \cdots, k+2 l+j\} \\
& \left\{u_{i}, v_{i}(J+1 \leqslant i \leqslant J+I), x\right\} \\
& \quad=\{k+2 l+j+1, k+2 l+j+2, \cdots, k+2 l+m=n\} .
\end{aligned}
$$

For brevity, we denote the collection $(U, V, w, x)$ simply by $\mathfrak{H}$.

After these preparations, we can give our main result in this paper as follows.

THEOREM 5. Let $\pi_{A}$ be the character of a discrete series representations such that the corresponding function $\tilde{\kappa}^{n, 0}$ on $H^{n, 0}$ is given by (7.2). Then, for every $H_{j}^{k, l}$, the function $\tilde{\kappa}_{j}^{k, l}$ on $F_{+} \subset H_{j}^{k, l}$ corresponding to $\pi_{A}$ is given by the following formula:

$$
\begin{aligned}
\tilde{\kappa}_{j}^{k, l}(h)= & (-1)^{n(n+1) / 2} \sum_{\mathfrak{Q}} \operatorname{sgn}(\mathfrak{U}) \xi_{k}\left(\delta_{1}, \delta_{2}, \cdots, \delta_{k} ; \lambda_{a_{1}}, \lambda_{a_{2}}, \cdots, \lambda_{a_{k}}\right) \\
& \times \prod_{1 \leqslant i \leqslant l} \eta_{2}\left(\delta_{k+2 i-1}, \delta_{k+2 i} ; \lambda_{b_{i}}, \lambda_{c_{i}}\right) \\
& \times \sum_{\mathfrak{u}} \prod_{1 \leqslant i \leqslant J+I} \zeta_{2}\left(\delta_{u_{i}}, \delta_{v_{i}} ; \lambda_{p_{i}}, \lambda_{q_{i}}\right) \cdot \chi\left(\delta_{w}, \delta_{x} ; \lambda_{r}, \lambda_{s}\right),
\end{aligned}
$$

where the first sum runs over all $\mathfrak{U}=(A, B, C, P, Q, r, s)$ satisfying the conditions (7.3)-(7.8), and the second sum runs over all $\mathfrak{U}=(U, V, w, x)$ satisfying the conditions (7.11)-(7.13), and $\xi_{k}=1$ for $k=0$.

Note that when $\nu_{1}, \nu_{2}, \cdots, \nu_{n}$ are all (or all except one) of the same sign, the above formula of $\tilde{\kappa}_{j}^{k, l}$ can be reduced to more simple form, as will be seen in $\S 9$. But the theorem gives us a unified formula of $\tilde{\kappa}_{j}^{k, l}$ valid for all A. In other words, if we consider $\tilde{\kappa}_{j}^{k, l}$ as a function not only of $\delta_{1}, \delta_{2}, \cdots, \delta_{n}$ 
but also of $\lambda_{1}=\nu_{1} l_{1}, \lambda_{2}=\nu_{2} l_{2}, \cdots, \lambda_{n}=\nu_{n} l_{n}$, then the formula (7.14) is the best possible, if we admit the functions of the form $\zeta_{2}$ as fundamental elements. In fact, put

$$
\begin{aligned}
E(\mathfrak{U}, \mathfrak{U})= & \xi_{k}\left(\delta_{1}, \delta_{2}, \cdots, \delta_{k} ; \lambda_{a_{1}}, \lambda_{a_{2}}, \cdots, \lambda_{a_{k}}\right) \\
& \times \prod_{1 \leqslant i \leqslant l} \eta_{2}\left(\delta_{k+2 i-1}, \delta_{k+2 i} ; \lambda_{b_{i}}, \lambda_{c_{i}}\right) \\
& \times \prod_{1 \leqslant i \leqslant J+I} \zeta_{2}\left(\delta_{u_{i}}, \delta_{v_{i}} ; \lambda_{p_{i}}, \lambda_{q_{i}}\right) \cdot \chi\left(\delta_{w}, \delta_{x} ; \lambda_{r}, \lambda_{s}\right)
\end{aligned}
$$

and consider it as a function of $\delta=\left(\delta_{1}, \delta_{2}, \cdots, \delta_{n}\right)$ and $\Lambda=\left(\lambda_{1}, \lambda_{2}, \cdots, \lambda_{n}\right)$. Then we see that these $\Xi(\mathfrak{U}, \mathfrak{U})$ 's are linearly independent mutually.

Note 1. A significance of $B, C, r$ etc. can be seen from the following identity. Let $N$ be a positive integer and put $l=[N / 2]$. A symbol $d$ denotes an integer when $N=2 l+1$, and the empty set $\varnothing$ when $N=2 l$.

$$
\begin{aligned}
& \operatorname{det}\left(\alpha_{i j}\right)_{1 \leqslant i, j \leqslant N} \\
& \quad=\sum \operatorname{sgn}(B \cdot C, d) \cdot D\left(\begin{array}{ll}
1 & 2 \\
b_{1} & c_{1}
\end{array}\right) D\left(\begin{array}{ll}
3 & 4 \\
b_{2} & c_{2}
\end{array}\right) \cdots D\left(\begin{array}{l}
N \\
d
\end{array}\right),
\end{aligned}
$$

where

$$
D\left(\begin{array}{ll}
i & i^{\prime} \\
j & j^{\prime}
\end{array}\right)=\left|\begin{array}{cc}
\alpha_{i j} & \alpha_{i j^{\prime}} \\
\alpha_{i^{\prime} j} & \alpha_{i^{\prime} j^{\prime}}
\end{array}\right|, \quad D\left(\begin{array}{l}
i \\
j
\end{array}\right)=\alpha_{i j},
$$

and the sum runs over all $(B, C, d)$ satisfying the condition (7.5) and

$$
\bar{B} \cup \bar{C} \cup\{d\}=\{1,2, \cdots, N\}=\Omega_{N} .
$$

Note 2. The formulas in Theorem 5 give us a tempered invariant eigendistributions even for $l_{1} \geqslant l_{2} \geqslant \cdots \geqslant l_{n} \geqslant 0$. In this case, it is better to denote $\xi_{k}\left(\cdot ; \nu_{1} l_{1}, \nu_{2} l_{2}, \cdots, \nu_{k} l_{k}\right)$ and $\zeta_{2}\left(\cdot ; \nu_{1} l_{1}, \nu_{2} l_{2}\right)$ by $\xi_{k}^{\prime}\left(\cdot ; l_{1}, l_{2}, \cdots, l_{k} ; \nu_{1}, \nu_{2}, \cdots, \nu_{k}\right)$ and $\zeta_{2}^{\prime}\left(\cdot ; l_{1}, l_{2} ; \nu_{1}, \nu_{2}\right)$ respectively, because some ambiguities occur for $l_{k}=0$ and $l_{2}=0$ respectively. For example, for $l_{1} \geqslant l_{2} \geqslant 0$, we put

$$
\zeta_{2}^{\prime}\left(\delta_{1}, \delta_{2} ; l_{1}, l_{2} ; \nu_{1}, \nu_{2}\right)=\left|\begin{array}{cc}
-\delta_{1}^{-l_{1}} & -\delta_{1}^{-l_{2}} \\
-\delta_{2}^{-l_{1}} & -\nu_{1} \nu_{2}\left(\delta_{2}^{-l_{2}}+\delta_{2}^{l_{2}}\right)+\delta_{2}^{l_{2}}
\end{array}\right| .
$$

Note that such a distribution is not necessarily zero even when $\tilde{\kappa}^{n, 0}$ in (7.2) is equal to zero. Moreover the proof of Theorem 5 given below remains valid for these degenerate cases too.

\section{§8. Proof of the main theorem}

In this section we give a proof of Theorem 5 . To this end, it is sufficient 
to prove that the system of functions $\left\{\tilde{\kappa}_{j}^{k, l}\right\}$ given by the formula (7.14) satisfies the conditions (T), (I), (II) and (III) in Theorem 4.

8.1. The condition (T). Since $\tilde{\kappa}_{j}^{k, l}$ is an integral linear combination of $\Xi(\mathfrak{U}, \mathfrak{H})$ 's in $(7.15)$, it is sufficient for the condition $(\mathrm{T})$ to prove that every $\Xi(\mathfrak{U}, \mathfrak{U})$ is bounded on $F_{+} \subset H_{j}^{k, l}$.

First consider the factor $D=\eta_{2}\left(\delta_{k+2 i-1}, \delta_{k+2 i} ; \lambda_{b_{i}}, \lambda_{c_{i}}\right)$. Recall that $\delta_{k+2 i-1}$ $=e^{\tau_{i}+\theta_{i}}, \delta_{k+2 i}=e^{\tau_{i}-\theta_{i}}, \lambda_{s}=\nu_{s} l_{s}$, and moreover that $\tau_{i}>0$ on $F_{+}$, and $l_{b_{i}}>l_{c_{i}}>0$ because $b_{i}<c_{i}$ by (7.5). Then we get from (6.2) that

$$
|D| \leqslant 2 \exp \left\{-\left(l_{b_{i}}+\nu_{b_{i}} \nu_{c_{i}} l_{c_{i}}\right) \tau_{i}\right\} \leqslant 2 .
$$

Next consider the factor $E=\zeta_{2}\left(\delta_{u_{i}}, \delta_{v_{i}} ; \lambda_{p_{i}}, \lambda_{q_{i}}\right)$. Recall that $\delta_{u_{i}}=$ $\varepsilon_{u_{i}} \exp t_{u_{i}}, \delta_{v_{i}}=\varepsilon_{v_{i}} \exp t_{v_{i}}$, and that $t_{u_{i}}>t_{v_{i}}>0$ on $F_{+}$because $u_{i}<v_{i}$ by (7.11), and $l_{p_{i}}>l_{q_{i}}>0$ because $p_{i}<q_{i}$ by (7.6). Then we get from (6.3) that

$$
\begin{aligned}
|E| \leqslant & \exp \left\{-\left(l_{p_{i}} t_{u_{i}}+l_{q_{i}} t_{v_{i}}\right)\right\}+\exp \left\{-\left(l_{q_{i}} t_{u_{i}}+l_{p_{i}} t_{v_{i}}\right)\right\} \\
& +2 \exp \left(-l_{p_{i}} t_{u_{i}}+l_{q_{i}} t_{v_{i}}\right) \leqslant 4 .
\end{aligned}
$$

Analogously we see that $\chi\left(\delta_{w}, \delta_{x} ; \lambda_{r}, \lambda_{s}\right)$ is bounded by 2 on $F_{+}$. Therefore we see that $\Xi(\mathfrak{U}, \mathfrak{H})$ in $(7.15)$ is bounded by a constant on $F_{+}$. Thus we proved that $\tilde{\kappa}_{j}^{k, l}$ is bounded on $F_{+}$, and so the condition $(\mathrm{T})$.

8.2. The condition (I). Since $\xi_{k}\left(\delta_{1}, \delta_{2}, \cdots, \delta_{k} ; \lambda_{a_{1}}, \lambda_{a_{2}}, \cdots, \lambda_{a_{k}}\right)$ is skewsymmetric in $\delta_{1}=e^{\Phi_{1}}, \delta_{2}=e^{\Phi_{2}}, \cdots, \delta_{k}=e^{\Phi_{k}}$, so is $\tilde{\kappa}_{j}^{k, l}$ itself. This proves (a) in the condition (I).

For an element $w$ in $\widetilde{S}_{l}$, put

$$
B^{w}=\left(b_{w(1)}, b_{w(2)}, \cdots, b_{w(l)}\right) .
$$

Then $\left(A, B^{w}, C^{w}, P, Q, r, s\right)$ satisfies the conditions (7.3)-(7.8) along with ( $A, B, C, P, Q, r, s)$, and

$$
\operatorname{sgn}\left(A, B^{w} \cdot C^{w}, P \cdot Q, r, s\right)=\operatorname{sgn}(A, B \cdot C, P \cdot Q, r, s) .
$$

Therefore it follows from (7.14) that $\tilde{\kappa}_{j}^{k, l}$ is invariant under any permutation of the pairs $\left(z_{i}, \bar{z}_{i}\right)(1 \leqslant i \leqslant l)$. This proves (b) in the condition (I).

We see from (6.2) that $\eta_{2}\left(\delta_{k+2 i-1}, \delta_{k+2 i} ; \lambda_{b_{i}}, \lambda_{c_{i}}\right)$ with $\delta_{k+2 i-1}=e^{z_{i}+\theta_{i}}, \delta_{k+2 i}=$ $e^{\tau_{i}-\theta_{i}}$ is odd in $\Theta_{i}$. Hence so is $\tilde{\kappa}_{j}^{k, l}$ and this proves (c) in the condition (I).

8.3. The condition (II). The function $\Xi(\mathfrak{U}, \mathfrak{U})$ in (7.15), and so the function $\tilde{\kappa}_{j}^{k, l}$ are integral linear combinations of the functions of the form

$$
\xi=\delta_{1}^{\mu_{1}} \delta_{2}^{\mu_{2}} \cdots \delta_{n}^{\mu_{n}}=\exp \left(\mu_{1} x_{1}+\mu_{2} x_{2}+\cdots+\mu_{n} x_{n}\right)
$$

where $\mu_{i}= \pm \lambda_{w(i)}$ for some $w \in \mathbb{S}_{n}$ and the co-ordinates $\left(x_{1}, x_{2}, \cdots, x_{n}\right)$ are 
defined by (5.2). We see that $\xi$ satisfies

$$
P\left(\frac{\partial}{\partial x_{1}}, \frac{\partial}{\partial x_{2}}, \cdots, \frac{\partial}{\partial x_{n}}\right) \xi=P\left(\lambda_{1}, \lambda_{2}, \cdots, \lambda_{n}\right) \xi
$$

for any symmetric polynomial $P\left(Z_{1}, Z_{2}, \cdots, Z_{n}\right)$ of $Z_{1}^{2}, Z_{2}^{2}, \cdots, Z_{n}{ }^{2}$. Therefore $\tilde{\kappa}_{j}^{k, l}$ satisfies the same equations, and hence the condition (II).

8.4. The condition (III), Case (1). Let us study the equations (4.11), (4.12) corresponding to $H_{j}^{k, l} \stackrel{\alpha, g}{\longrightarrow} H_{j}^{k+2, l-1}$. We follow the direction of the idea in $\S 5.2$. Then $\tilde{\kappa}_{j}^{k, l}$ comes into question only modulo even functions in $\tau_{p}$. We apply the following lemma whose proof is omitted here.

LEMMA 8.1. Put $\lambda_{1}=\nu_{1} l_{1}, \lambda_{2}=\nu_{2} l_{2}$ and $z=\tau+\Theta, \bar{z}=\tau-\Theta(\Theta=\sqrt{-1} \theta) ; \Phi_{1}=$ $\Psi+\Theta, \Phi_{2}=\Psi-\Theta(\Psi=\sqrt{-1} \psi)$. Then for $M=0,1,2, \cdots$,

$$
\left(\frac{\partial}{\partial \tau}\right)_{0}^{2 M+1} \eta_{2}\left(e^{z}, e^{\bar{z}} ; \lambda_{1}, \lambda_{2}\right)=\left(\frac{\partial}{\partial \Psi}\right)_{0}^{2 M+1} \xi_{2}\left(e^{\Phi_{1}}, e^{\Phi_{2}} ; \lambda_{1}, \lambda_{2}\right)
$$

where $(\partial / \partial \tau)_{0}$ denotes the differentiation at $\tau=0$.

Now put

$$
\Phi_{k+1}=\Psi+\Theta_{p}, \quad \Phi_{k+2}=\Psi-\Theta_{p} \quad(\Psi=\sqrt{-1} \psi)
$$

and

$$
\begin{aligned}
\eta= & (-1)^{n(n+1) / 2} \sum_{\mathfrak{U}} \operatorname{sgn}(\mathfrak{U}) \xi_{k}\left(\delta_{1}, \delta_{2}, \cdots, \delta_{k} ; \lambda_{a_{1}}, \lambda_{a_{2}}, \cdots, \lambda_{a_{k}}\right) \\
& \times \xi_{2}\left(e^{\Phi_{k+1}}, e^{\Phi_{k+2}} ; \lambda_{b_{p}}, \lambda_{c_{p}}\right) \cdot \prod_{i \neq p} \eta_{2}\left(\delta_{k+2 i-1}, \delta_{k+2 i} ; \lambda_{b_{i}}, \lambda_{c_{i}}\right) \\
& \times \sum_{\mathfrak{u}} \prod_{1 \leqslant i \leqslant J+I} \zeta_{2}\left(\delta_{u_{i}}, \delta_{v_{i}} ; \lambda_{p_{i}}, \lambda_{q_{i}}\right) \cdot \chi\left(\delta_{w}, \delta_{x} ; \lambda_{r}, \lambda_{s}\right),
\end{aligned}
$$

where the sums are taken over $\mathfrak{U}=(A, B, C, P, Q, r, s)$ and $\mathfrak{U}=(U, V, w, x)$ respectively just as for (7.14). Then by Lemma 8.1 above, we get

$$
\left(\frac{\partial}{\partial \tau_{p}}\right)_{0}^{2 M+1} \tilde{\kappa}_{j}^{k, l}=\left(\frac{\partial}{\partial \Psi}\right)_{0}^{2 M+1} \eta \quad(M=0,1,2, \cdots) .
$$

Therefore, in order to prove that $\tilde{\kappa}_{j}^{k . l}$ and $\tilde{\kappa}_{j}^{k+2, l-1}$ satisfy the equations (4.11), (4.12), it is sufficient to see that the following equality holds:

$$
\begin{gathered}
\tilde{\kappa}_{j}^{k+2, l-1}=\left.\eta\right|_{\left(8.5^{\prime}\right)}, \\
z_{q} \rightarrow z_{q-1}, \quad \bar{z}_{q} \rightarrow \bar{z}_{q-1} \quad(p<q \leqslant l),
\end{gathered}
$$

where $\left.\right|_{\left(8.5^{\prime}\right)}$ means that the changes of the suffices of the variables in $\left(8.5^{\prime}\right)$ 
are carried out. In other words, put $\delta^{\prime}=\left(\delta_{1}^{\prime}, \delta_{2}^{\prime}, \cdots, \delta_{n}^{\prime}\right)$ as

$$
\begin{cases}\delta_{k+1}^{\prime}=e^{\Phi_{k+1}}, \quad \delta_{k+2}^{\prime}=e^{\Phi_{k+2}} ; & \\ \left(\delta_{k+2 i+1}^{\prime}, \delta_{k+2 i+2}^{\prime}\right)=\left(\delta_{k+2 i-1}, \delta_{k+2 i}\right) & \text { for } 1 \leqslant i \leqslant p: \\ \delta_{i}^{\prime}=\delta_{i} & \text { otherwise, }\end{cases}
$$

and let $h^{\prime}$ be the element in $H_{j}^{k+2, l-1}$ with the co-ordinates $\delta^{\prime}$. Then the equality (8.5) is expressed as

$$
\tilde{\kappa}_{j}^{k+2, l-1}\left(h^{\prime}\right)=\eta
$$

Let us prove this equality. For any $\mathfrak{A}=(A, B, C, P, Q, r, s)$ satisfying (7.3)-(7.8), we put

$$
\begin{aligned}
& B^{\prime}=\left(b_{1}^{\prime}, b_{2}^{\prime}, \cdots, b_{l-1}^{\prime}\right)=\left(b_{1}, b_{2}, \cdots, \hat{b}_{p}, \cdots, b_{l}\right), \\
& C^{\prime}=\left(c_{1}^{\prime}, c_{2}^{\prime}, \cdots, c_{l-1}^{\prime}\right)=\left(c_{1}, c_{2}, \cdots, \hat{c}_{p}, \cdots, c_{l}\right),
\end{aligned}
$$

where $\hat{b}_{p}$ indicates that $b_{p}$ is absent. Then

$$
\operatorname{sgn}(A, B \cdot C, P \cdot Q, r, s)=\operatorname{sgn}\left(A, b_{p}, c_{p}, B^{\prime} \cdot C^{\prime}, P \cdot Q, r, s\right) .
$$

Let $A^{\prime}=\left(a_{1}^{\prime}, a_{2}^{\prime}, \cdots, a_{k+2}^{\prime}\right)$ be the ordered set of all integers in $\Omega_{n}-\left(\bar{B}^{\prime} \cup \bar{C}^{\prime} \cup\right.$ $\bar{P} \cup \bar{Q} \cup\{r, s\})$ such that

$$
a_{1}^{\prime}<a_{2}^{\prime}<\ldots<a_{k+2}^{\prime}
$$

and put $\mathfrak{Y}^{\prime}=\left(A^{\prime}, B^{\prime}, C^{\prime}, P, Q, r, s\right)$. Then we get

$$
\begin{aligned}
& \operatorname{sgn}\left(A, b_{p}, c_{p}, B^{\prime} \cdot C^{\prime}, P \cdot Q, r, s\right) \\
& \quad=\operatorname{sgn}\left(A^{\prime}, B^{\prime} \cdot C^{\prime}, P \cdot Q, r, s\right) \cdot \operatorname{sgn}\left(A^{\prime} ; A, b_{p}, c_{p}\right),
\end{aligned}
$$

and so by (8.9)

$$
\operatorname{sgn}(\mathfrak{U})=\operatorname{sgn}\left(\mathfrak{H}^{\prime}\right) \cdot \operatorname{sgn}\left(A^{\prime} ; A, b_{p}, c_{p}\right),
$$

where $\operatorname{sgn}\left(A^{\prime} ; A, b_{p}, c_{p}\right)$ denotes the sign of the permutation $\left(A, b_{p}, c_{p}\right)$ of $A^{\prime}$.

Now fix $B^{\prime}, C^{\prime}, P, Q, r$ and $s$ in $\mathscr{Y}$. Taking the sum over all possible $\left(A, b_{p}, c_{p}\right)$, we get the following equality:

$$
\begin{aligned}
\sum \operatorname{sgn}( & \left.A^{\prime} ; A, b_{p}, c_{p}\right) \xi_{k}\left(\delta_{1}, \delta_{2}, \cdots, \delta_{k} ; \lambda_{a_{1}}, \lambda_{a_{2}}, \cdots, \lambda_{a_{k}}\right) \\
& \times \xi_{2}\left(e^{\Phi_{k+1}}, e^{\Phi_{k+2}} ; \lambda_{b_{p}}, \lambda_{c_{p}}\right) \\
= & \xi_{k+2}\left(\delta_{1}, \delta_{2}, \cdots, \delta_{k}, e^{\Phi_{k+1}}, e^{\Phi_{k+2}} ; \lambda_{a_{1}}, \lambda_{a_{2}^{\prime}}, \cdots, \lambda_{a_{k+2}^{\prime}}\right) .
\end{aligned}
$$

Using (8.10) and (8.11), we can rewrite the sum over $\mathfrak{X}$ in (8.3) as the sum 
over $\mathfrak{U}^{\prime}$, and then obtain the equality (8.7). Thus the condition (III) is now proved in Case (1).

8.5. The condition (III), Case (2+). Let us study the equations (4.21), (4.22) corresponding to $H_{j}^{k, l} \stackrel{\alpha, g}{\longrightarrow} H_{j-1}^{k+1, l}$. To this end, in the direction of the idea in $\S 5.2$, it is sufficient to consider the function $\tilde{\kappa}_{j}^{k, l}$ modulo even functions in the variable $t_{j}$. We apply the following two lemmas to the Cases $\left(2^{+}\right)$and $\left(2^{-}\right)$.

LEMMA 8.2. Let $\varepsilon= \pm 1$ and $\lambda_{1}=\nu_{1} l_{1}, \lambda_{2}=\nu_{2} l_{2}$. Then there hold the following equalities modulo even functions in $t_{2}$ :

$$
\zeta_{2}\left(\varepsilon e^{t_{1}}, \varepsilon e^{t_{2}} ; \lambda_{1}, \lambda_{2}\right) \equiv \chi\left(\varepsilon e^{t_{1}}, \varepsilon e^{t_{2}} ; \lambda_{1}, \lambda_{2}\right) .
$$

LEMMA 8.3. Let $\varepsilon, \lambda_{1}$ and $\lambda_{2}$ be as above, then for $M=0,1,2, \cdots$,

$$
\begin{aligned}
& \left(\frac{\partial}{\partial t_{1}}\right)_{0}^{2 M+1} \zeta_{2}\left(\varepsilon e^{t_{1}}, \varepsilon e^{t_{2}} ; \lambda_{1}, \lambda_{2}\right)=\left(\frac{\partial}{\partial t_{1}}\right)_{0}^{2 M+1} \chi\left(\varepsilon e^{t_{1}}, \varepsilon e^{t_{2}} ; \lambda_{1}, \lambda_{2}\right) \\
& =\left(\frac{\partial}{\partial \Phi}\right)_{(1-\varepsilon) \pi i / 2}^{2 M+1}\left\{\xi_{1}\left(e^{\Phi} ; \lambda_{1}\right) \cdot \chi\left(\varepsilon e^{t_{2}}, \lambda_{2}\right)-\xi_{1}\left(e^{\Phi} ; \lambda_{2}\right) \cdot \chi\left(\varepsilon e^{t_{2}} ; \lambda_{1}\right)\right\} ; \\
& \left(\frac{\partial}{\partial t_{1}}\right)_{0}^{2 M+1} \chi\left(\varepsilon e^{t_{1}} ; \lambda_{1}\right)=\left(\frac{\partial}{\partial \Phi}\right)_{(1-\varepsilon) \pi i / 2}^{2 M+1} \xi_{1}\left(e^{\Phi} ; \lambda_{1}\right) \quad(i=\sqrt{-1}) .
\end{aligned}
$$

Note that we put $\varepsilon=1$ for Case $\left(2^{+}\right)$, and $\varepsilon=-1$ for Case $\left(2^{-}\right)$in the next. subsection. Now we must treat the two cases $j=2 J+1, j=2 J$ separately.

(1) The case $j=2 J+1$. In this case the symbol $r$ and $w$ both denote integers. Put $\bar{j}=k+2 l+j$, then $\delta_{j}=e^{t_{j}}$. Devide the sum over $\mathfrak{H}=(U, V, w$, $x$ ) in (7.14) into two parts: the one for which $w=\bar{j}$ and the other for which $w<\bar{j}$ (in the latter, $v_{J}=\bar{j}$ by (7.12) and (7.13)). Corresponding to these two parts, we put

$$
\begin{aligned}
& \zeta^{1}(P, Q, r, s) \\
& =\sum_{\mathfrak{u}: w=j} \prod_{1 \leqslant i \leqslant J+I} \zeta_{2}\left(\delta_{u_{i}}, \delta_{v_{i}} ; \lambda_{p_{i}}, \lambda_{q_{i}}\right) \cdot \chi\left(\delta_{w}, \delta_{x} ; \lambda_{r}, \lambda_{s}\right), \\
& \zeta^{2}(P, Q, r, s)=\sum_{\mathfrak{u}: w<j\left(v_{J}=j\right)} \prod_{i \neq J} \zeta_{2}\left(\delta_{u_{i}}, \delta_{v_{i}} ; \lambda_{p_{i}}, \lambda_{q_{i}}\right) \\
& \times \chi\left(\delta_{u_{J}}, \delta_{j} ; \lambda_{p_{J}}, \lambda_{q_{J}}\right) \cdot \chi\left(\delta_{w}, \delta_{x} ; \lambda_{r}, \lambda_{s}\right),
\end{aligned}
$$

and then put

$$
\begin{aligned}
\xi= & (-1)^{n(n+1) / 2} \sum_{\mathscr{A}} \operatorname{sgn}(\mathfrak{U}) \xi_{k}\left(\delta_{1}, \delta_{2}, \cdots, \delta_{k} ; \lambda_{a_{1}}, \lambda_{a_{2}}, \cdots, \lambda_{a_{k}}\right) \\
& \times \prod_{1 \leqslant i \leqslant l} \eta_{2}\left(\delta_{k+2 i-1}, \delta_{k+2 i} ; \lambda_{b_{i}}, \lambda_{c_{i}}\right)\left\{\zeta^{1}(P, Q, r, s)+\zeta^{2}(P, Q, r, s)\right\},
\end{aligned}
$$


where $\mathfrak{A}$ denotes $(A, B, C, P, Q, r, s)$ as before. Then we get from Lemma 8.2 that

$$
\tilde{\kappa}_{j}^{k, l} \equiv \xi \quad \text { modulo even functions in } t_{j} .
$$

This means, by what was said above, that it is sufficient here to treat $\xi$ instead of $\tilde{\kappa}_{j}^{k, l}$. Now we apply the following lemma.

LEMMA 8.4. Fix $A, B$ and $C$. Then

$$
\sum_{(P, Q, r, s)} \operatorname{sgn}(\mathscr{Y}) \zeta^{2}(P, Q, r, s)=0,
$$

where the sum runs over all possible $(P, Q, r, s)$.

Proof. Fix the ordered pairs $\left(p_{i}, q_{i}\right)$ 's except $\left(p_{J}, q_{J}\right)$, and put $\Omega=$ $\left\{p_{J}, q_{J}, r, s\right\}$. Then $\Omega$ is a fixed subset of $\Omega_{n}$.

When $m-j=2 I$, the symbols $s$ and $x$ both denote $\varnothing$. Arrange the elements in $\Omega$ as $d_{1}<d_{2}<d_{3}$ and put $D=\left(d_{1}, d_{2}, d_{3}\right)$. Then

$$
\begin{gathered}
\sum_{\left(p_{J}, q_{J}, r\right)} \operatorname{sgn}\left(D ; p_{J}, q_{J}, r\right) \cdot \chi\left(\delta_{u_{J}}, \delta_{j} ; \lambda_{p_{J}}, \lambda_{q_{J}}\right) \cdot \chi\left(\delta_{w} ; \lambda_{r}\right) \\
=1-\delta^{-\mu_{1}}-\delta^{\mu_{2}}-\left.\delta^{\mu_{3}}\right|_{\delta=\delta_{u_{J}, \delta_{j}, \delta_{w}}}
\end{gathered}
$$

where $\left(\mu_{1}, \mu_{2}, \mu_{3}\right)=\left(l_{d_{1}}, l_{d_{2}}, l_{d_{3}}\right)$ and the sum runs over all $\left(p_{J}, q_{J}, r\right)$ such that

$$
\left\{p_{J}, q_{J}, r\right\}=\Omega, \quad p_{J}<q_{J} .
$$

When $m-j=2 I+1$, the symbols $s$ and $x$ both denote integers. Arrange the elements in $\Omega$ as $d_{1}<d_{2}<d_{3}<d_{4}$ and put $D=\left(d_{1}, d_{2}, d_{3}, d_{4}\right)$. Then

$$
\begin{gathered}
\sum_{\left(p_{J}, q_{J}, r, s\right)} \operatorname{sgn}\left(D ; p_{J}, q_{J}, r, s\right) \cdot \chi\left(\delta_{u_{J}}, \delta_{j} ; \lambda_{p_{J}}, \lambda_{q_{J}}\right) \cdot \chi\left(\delta_{w}, \delta_{x} ; \lambda_{r}, \lambda_{s}\right) \\
=1-\delta^{-\mu_{1}}-\delta^{-\mu_{2}}-\left.\delta^{-\mu_{3}} \delta^{-\mu_{4}}\right|_{\delta=\delta_{u_{J}}, \hat{\delta}_{j}, \delta_{w}, \delta_{x}},
\end{gathered}
$$

where $\left(\mu_{1}, \mu_{2}, \mu_{3}, \mu_{4}\right)=\left(l_{d_{1}}, l_{d_{2}}, l_{d_{3}}, l_{d_{4}}\right)$ and the sum runs over all $\left(p_{J}, q_{J}, r, s\right)$ such that

$$
\left\{p_{J}, q_{J}, r, s\right\}=\Omega, \quad p_{J}<q_{J}, \quad r<s .
$$

Since $v_{J}=\bar{j}$, we may exchange the rolls of $u_{J}$ and $w$ mutually under the conditions (7.14)-(7.16). The partial sum in (8.16) or that in (8.17) over $\left(u_{J}, w\right)=(\alpha, \beta),(\beta, \alpha)$ is equal to zero in respective cases. Thus we get the equality in the lemma in both cases.

Q.E.D.

By this lemma, the term $\zeta^{2}$ in (8.14) disappears. Now put

$$
Y_{w, x}=\xi_{1}\left(e^{\Phi_{k+1}} ; \lambda_{r}\right) \chi\left(\delta_{x} ; \lambda_{s}\right)-\xi_{1}\left(e^{\Phi_{k+1}} ; \lambda_{s}\right) \chi\left(\delta_{x} ; \lambda_{r}\right),
$$


when $m-j=2 I+1$ (both $s$ and $x$ denote integers), and

$$
Y_{w, x}=\xi_{1}\left(e^{\Phi_{k+1}} ; \lambda_{r}\right),
$$

when $m-j=2 I$ (both $s$ and $x$ denote $\varnothing$ ). Moreover put

$$
\begin{aligned}
\eta= & (-1)^{n(n+1) / 2} \sum_{\mathfrak{U}} \operatorname{sgn}(\mathfrak{X}) \xi_{k}\left(\delta_{1}, \delta_{2}, \cdots, \delta_{k} ; \lambda_{a_{1}}, \lambda_{a_{2}}, \cdots, \lambda_{a_{k}}\right) \\
& \times \prod_{1 \leqslant i \leqslant l} \eta_{2}\left(\delta_{k+2 i-1}, \delta_{k+2 i} ; \lambda_{b_{i}}, \lambda_{c_{i}}\right) \\
& \times \sum_{\mathfrak{u}: w=j} \prod_{1 \leqslant i \leqslant J+I} \zeta_{2}\left(\delta_{u_{i}}, \delta_{v_{i}} ; \lambda_{p_{i}}, \lambda_{q_{i}}\right) \times Y_{w, x},
\end{aligned}
$$

then we get

$$
\left(\frac{\partial}{\partial t_{j}}\right)_{0}^{2 M+1} \tilde{\kappa}_{j}^{k, l}=\left(\frac{\partial}{\partial \Phi_{k+1}}\right)_{0}^{2 M+1} \eta \quad(M=0,1,2, \cdots) .
$$

Put $\delta^{\prime}=\left(\delta_{1}^{\prime}, \delta_{2}^{\prime}, \cdots, \delta_{n}^{\prime}\right)$ as

$$
\begin{cases}\delta_{k+1}^{\prime}=e^{\Phi_{k+1}}, \quad \delta_{i}^{\prime}=\delta_{i-1} & \text { for } k+2 \leqslant i \leqslant k+2 l+j, \\ \delta_{i}^{\prime}=\delta_{i} & \text { otherwise, }\end{cases}
$$

and let $h^{\prime} \in H_{j-1}^{k+1, l}$ be the element with the co-ordinates $\delta^{\prime}$. We wish to prove the equality

$$
\tilde{\kappa}_{j-1}^{k+1, l}\left(h^{\prime}\right)=\eta .
$$

Then as in the Case (1), the equalities (8.18), (8.20) imply that the equations (4.21), (4.22) are satisfied in the case $j=2 J+1$ (in this case $(-1)^{j-1}=1$ ).

Let us prove (8.20). Fix $B, C, P, Q$ and $s$, and put $\Omega=\bar{A} \cup\{r\}$. Then $\Omega$ is a fixed subset of $\Omega_{n}$. Let $A^{\prime}=\left(a_{1}^{\prime}, a_{2}^{\prime}, \cdots, a_{k+1}^{\prime}\right)$ be the ordered set of elements in $\Omega$ such that $a_{1}^{\prime}<a_{2}^{\prime}<\ldots<a_{k+1}^{\prime}$. Then summing up over all possible $(A, r)$, we get

$$
\begin{gathered}
\sum \operatorname{sgn}\left(A^{\prime} ; A, r\right) \cdot \xi_{k}\left(\delta_{1}, \delta_{2}, \cdots, \delta_{k} ; \lambda_{a_{1}}, \lambda_{a_{2}}, \cdots, \lambda_{a_{k}}\right) \cdot \xi_{1}\left(e^{\Phi_{k+1}} ; \lambda_{r}\right) \\
=\xi_{k+1}\left(\delta_{1}, \delta_{2}, \cdots, \delta_{k}, e^{\Phi_{k+1}} ; \lambda_{a_{1}^{\prime}}, \lambda_{a_{2}^{\prime}}, \cdots, \lambda_{a_{k+1}^{\prime}}\right) .
\end{gathered}
$$

When $m-j=2 I$ and so $s$ denotes $\varnothing$, this equality is sufficient to prove (8.20). When $m-j=2 I+1$ and so $s$ denotes an integer, we need one more equality obtained as follows. Fix $B, C, P, Q$ and $r$, and put $\Omega=\bar{A} \cup\{s\}$. Let $A^{\prime}$ be as above, then the analogous equality as (8.21) holds for the sum over $(A, s)$ 's.

Now taking into account the equality

$$
\begin{aligned}
\operatorname{sgn}(A, B \cdot C, P \cdot Q, r, s) & =\operatorname{sgn}(A, r, B \cdot C, P \cdot Q, \varnothing, s) \\
& =\operatorname{sgn}(A, s, B \cdot C, P \cdot Q, \varnothing, r)
\end{aligned}
$$


and the similar one as (8.10), we see from the above arguments that $\eta$ is always equal to $\tilde{\kappa}_{j-1}^{k+1, l}$ irrespective of the parity of $m-j$. Thus the equality is now proved.

(2) The case $j=2 J$. In this case, the symbols $r$ and $w$ denote $\varnothing$, and $v_{J}=\bar{j}$ always. We define anew a function $\eta$ only by replacing in (7.14) $\zeta_{2}\left(\delta_{u_{J}}, \delta_{v_{J}}=\delta_{j} ; \lambda_{p_{J}}, \lambda_{q_{J}}\right)$ by

$$
\chi\left(\delta_{u_{J}} ; \lambda_{p_{J}}\right) \xi_{1}\left(e^{\Phi_{k+1}} ; \lambda_{q_{J}}\right)-\chi\left(\delta_{u_{J}}, \lambda_{q_{J}}\right) \xi_{1}\left(e^{\Phi_{k+1}} ; \lambda_{p_{J}}\right) .
$$

Then by Lemma 8.3 we get

$$
\left(\frac{\partial}{\partial t_{j}}\right)_{0}^{2 M+1} \tilde{\kappa}_{j}^{k, l}=\left(\frac{\partial}{\partial \Phi_{k+1}}\right)_{0}^{2 M+1} \eta \quad(M=0,1,2, \cdots) .
$$

Now we wish to prove the following equality:

$$
\tilde{\kappa}_{j}^{k+1, l}\left(h^{\prime}\right)=-\eta,
$$

where $h^{\prime}$ is just as for (8.20). Note that $(-1)^{j-1}=-1$ in the present case, then we see from the above two equalities that the equations (4.21), (4.22) are satisfied.

To prove (8.24), first consider the case $m-j=2 I$. Then the symbols $s$ denotes $\varnothing$. Fix $B, C,\left(p_{i}, q_{i}\right)$ 's except $\left(p_{J}, q_{J}\right)$ and put

$$
P^{\prime}=\left(p_{1}, p_{2}, \cdots, \hat{p}_{J}, \cdots, p_{J+I}\right), \quad Q^{\prime}=\left(q_{1}, q_{2}, \cdots, \hat{q}_{J}, \cdots, q_{J+I}\right) .
$$

Moreover put $\Omega=\bar{A} \cup\left\{p_{J}, q_{J}\right\}$, then $\Omega$ is a fixed subset of $\Omega_{n}$. Now fix an arbitrary element $r^{\prime}$ in $\Omega$, and let $A^{\prime}=\left(a_{1}^{\prime}, a_{2}^{\prime}, \cdots, a_{k+1}^{\prime}\right)$ be an ordered set of elements in $\Omega-\left\{r^{\prime}\right\}$ such that

$$
a_{1}^{\prime}<a_{2}^{\prime}<\cdots<a_{k+1}^{\prime}
$$

Take an element $a_{\alpha}^{\prime}$, and put anew $A=\left(a_{1}^{\prime}, a_{2}^{\prime}, \cdots, \hat{a}_{\alpha}^{\prime}, \cdots, a_{k+1}^{\prime}\right)$ and

$$
\left(p_{J}, q_{J}\right)= \begin{cases}\left(a_{\alpha}^{\prime}, r^{\prime}\right) & \text { if } a_{\alpha}^{\prime}<r^{\prime}, \\ \left(r^{\prime}, a_{\alpha}^{\prime}\right) & \text { if } \alpha_{\alpha}^{\prime}>r^{\prime} .\end{cases}
$$

Then they satisfy together with $B, C,\left(p_{i}, q_{i}\right)$ 's for $i \neq J, r=\varnothing, s=\varnothing$, the conditions (7.4)-(7.8). Thus we get the following equality :

$$
\begin{aligned}
\sum_{\left(A, p_{J}, q_{J}\right)} & \operatorname{sgn}(A, B \cdot C, P \cdot Q) \xi_{k}\left(\delta_{1}, \delta_{2}, \cdots, \delta_{k} ; \lambda_{a_{1}}, \lambda_{a_{2}}, \cdots, \lambda_{a_{k}}\right) \\
& \times\left\{\chi\left(\delta_{u_{J}} ; \lambda_{p_{J}}\right) \xi_{1}\left(e^{\Phi_{k+1}} ; \lambda_{q_{J}}\right)-\chi\left(\delta_{u_{J}} ; \lambda_{q_{J}}\right) \xi_{1}\left(e^{\Phi_{k+1}} ; \lambda_{p_{J}}\right)\right\} ; \\
= & -\sum_{\left(A^{\prime}, r^{\prime}\right.} \operatorname{sgn}\left(A^{\prime}, B \cdot C, P^{\prime} \cdot Q^{\prime}, r^{\prime}\right) \\
& \times \xi_{k+1}\left(\delta_{1}, \delta_{2}, \cdots, \delta_{k}, e^{\Phi_{k+1}} ; \lambda_{a_{1}^{\prime}}, \lambda_{a_{2}^{\prime}}, \cdots, \lambda_{a_{k+1}}\right) \cdot \chi\left(\delta_{u_{J}} ; \lambda_{r^{\prime}}\right),
\end{aligned}
$$


where the first sum runs over all possible $\left(A, p_{J}, q_{J}\right)$ such that $\bar{A} \cup\left\{p_{J}, q_{J}\right\}$ $=\Omega$, and the second one runs over all $\left(A^{\prime}, r^{\prime}\right)$ satisfying (8.25) and $\bar{A}^{\prime} \cup\left\{r^{\prime}\right\}$ $=\Omega$. We see from this equality that (8.24) holds when $m-j=2 I$.

The case $m-j=2 I+1$ can be treated similarly. Note that in this case the symbol $s$ denote an integer, and so we must use further the following equality :

$$
\chi\left(\delta_{1}, \delta_{2} ; \lambda_{1}, \lambda_{2}\right)=\chi\left(\delta_{1}, \lambda_{1}\right) \chi\left(\delta_{2} ; \lambda_{2}\right)-\chi\left(\delta_{1} ; \lambda_{2}\right) \chi\left(\delta_{2} ; \lambda_{1}\right) .
$$

Thus we proved the equality (8.24) irrespective of the parity of $m-j$. Therefore the condition (III) is now proved completely in the Case $\left(2^{+}\right)$.

8.6. The condition (III), Case (2-). The equations (4.23) corresponding to $H_{j}^{k, l} \stackrel{\alpha, g}{\longrightarrow} H_{j}^{k+1, l}$ can be studied quite analogously as the Case $\left(2^{+}\right)$in the preceeding subsection. Here we must employ $\left(\partial / \partial \Phi_{k+1}\right)_{\pi i}$ instead of $\left(\partial / \partial \Phi_{k+1}\right)_{0}$, and apply Lemmas 8.2 and 8.3 for $\varepsilon=-1$. The detailed calculations are omitted here.

8.7. The condition (III), Case $\left(3^{+}\right)$. Let us study the equations (4.35), (4.36) corresponding to $H_{j}^{k, l} \stackrel{\alpha, g}{\longrightarrow} H_{j-2}^{k, l+1}$. In this case, we put

$$
t_{p}=\tau_{l+1}+\sigma, \quad t_{p+1}=\tau_{l+1}-\sigma,
$$

where $p$ is as in $\S 4.5(p+1 \leqslant j)$, and study the function $\tilde{\kappa}_{j}^{k, l}$ modulo even functions in the variable $\sigma$ or equivalently modulo symmetric functions in $\left(t_{p}, t_{p+1}\right)$.

Fix $\mathfrak{A}=(A, B, C, P, Q, r, s)$ in (7.14) and consider the sum over $\mathfrak{U}=(U$, $V, w, x)$. Put $\bar{p}=k+2 l+p$, then $\delta_{\tilde{p}}=e^{t_{p}}, \delta_{\bar{p}+1}=e^{t_{p+1}}$. Let us first study the places of $\bar{p}$ and $\bar{p}+1$ in $(U, V, w, x)$. Recall that $v_{i}<v_{i^{\prime}}$ for $i<i^{\prime}<J$ by (7.12), and that $w \leqslant j=k+2 l+j$ by (7.13) when $w$ denotes an integer or $j=$ $2 J+1$. Then we have the following cases: denote by $i$ and $i^{\prime}$ two positive integers such that $i<i^{\prime} \leqslant J$,

( $\alpha) \quad\left(u_{i}, v_{i}\right)=(\bar{p}, \bar{p}+1)$;

(ß) $v_{i}=\bar{p}, v_{i^{\prime}}=\bar{p}+1$

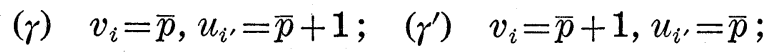

(ס) $u_{i}=\bar{p}, u_{i^{\prime}}=\bar{p}+1 ; \quad\left(\delta^{\prime}\right) \quad u_{i}=\bar{p}+1, u_{i^{\prime}}=\bar{p}$;

and moreover when $j=2 J+1$,

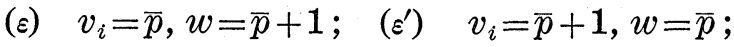

(ф) $\quad u_{i}=\bar{p}, w=\bar{p}+1 ; \quad\left(\phi^{\prime}\right) \quad u_{i}=\bar{p}+1, w=\bar{p}$.

Let us now consider the case ( $\beta$ ). In this case, $i^{\prime}=i+1$ because of (7.12). Therefore we see easily that we may exchange $u_{i}, u_{i+1}$ under the conditions (7.11)-(7.13) (all other elements in $(U, V, w, x)$ are left unchanged). 
Consider the following term in the sum in (7.14):

$$
\prod_{1 \leqslant \alpha \leqslant J+I} \zeta_{2}\left(\delta_{u_{\alpha}}, \delta_{v_{\alpha}} ; \lambda_{p_{\alpha}}, \lambda_{q_{\alpha}}\right) \cdot \chi\left(\delta_{w}, \delta_{x} ; \lambda_{r}, \lambda_{s}\right)
$$

Then the sum of two such terms corresponding to the above exchange of $u_{i}, u_{i+1}$ is symmetric in $\left(t_{p}, t_{p+1}\right)$, and so is equal to zero modulo symmetric functions.

Next consider the cases $(\gamma)$ and $\left(\gamma^{\prime}\right)$. Then both $(\gamma)$ and $\left(\gamma^{\prime}\right)$ exist at the same time when all other elements than $v_{i}, u_{i}$ are fixed in $\mathfrak{H}$. This means that we can exchange $v_{i}, u_{i^{\prime}}$ in this situation under the conditions (7.11)(7.13). Thus the sum of (8.27) corresponding to $(\gamma)$ and $\left(\gamma^{\prime}\right)$ is symmetric in $\left(t_{p}, t_{p+1}\right)$ and so equal to zero modulo symmetric functions.

The cases $(\delta)$ and $\left(\delta^{\prime}\right)$ etc. are similar as above. So we need not take . into account them here and then there rests only the case $(\alpha)$.

Let us consider the case $(\alpha)$. We replace in the summands in (7.14) corresponding to this case,

$$
\zeta_{2}\left(\delta_{u_{i}}, \delta_{v_{i}} ; \lambda_{p_{i}}, \lambda_{q_{i}}\right)=\zeta_{2}\left(\delta_{\bar{p}}, \delta_{\bar{p}+1} ; \lambda_{p_{i}}, \lambda_{q_{i}}\right) \quad\left(\delta_{\bar{p}}=e^{t_{p}}, \delta_{\bar{p}+1}=e^{t_{p+1}}\right)
$$

by

$$
\zeta_{2}\left(e^{z l+1}, e^{\bar{z}_{l+1}} ; \lambda_{p_{i}}, \lambda_{q_{i}}\right) \quad\left(z_{l+1}=\tau_{l+1}+\Theta_{l+1}\right) .
$$

Then we get a function $\eta$ which satisfies the following equations because of Lemma 8.5 given below :

$$
\left(\frac{\partial}{\partial \sigma}\right)_{0}^{2 M+1} \tilde{\kappa}_{j}^{k, l}=\left(\frac{\partial}{\partial \Theta_{l+1}}\right)_{0}^{2 M+1} \eta \quad(M=0,1,2, \cdots) .
$$

LEMMA 8.5. In the notations in (8.26) and (8.28), there hold the following equalities : for $M=0,1,2, \cdots$,

$$
\left(\frac{\partial}{\partial \sigma}\right)_{0}^{2 M+1} \zeta_{2}\left(e^{t_{p}}, e^{t_{p+1}} ; \lambda_{p_{i}}, \lambda_{q_{i}}\right)=\left(\frac{\partial}{\partial \Theta_{l+1}}\right)_{0}^{2 M+1} \eta_{2}\left(e^{z_{l+l}}, e^{\bar{z} l+1} ; \lambda_{p_{i}}, \lambda_{q_{i}}\right) .
$$

Now we wish to rewrite the expression of $\eta$ and obtain the analogous equality as (8.20) (see (8.33) below). Put

$$
\begin{aligned}
& B^{\prime}=\left(b_{1}^{\prime}, b_{2}^{\prime}, \cdots, b_{l+1}^{\prime}\right)=\left(b_{1}, b_{2}, \cdots, b_{l}, p_{i}\right), \\
& C^{\prime}=\left(c_{1}^{\prime}, c_{2}^{\prime}, \cdots, c_{l+1}^{\prime}\right)=\left(c_{1}, c_{2}, \cdots, c_{l}, q_{i}\right) ; \\
& P^{\prime}=\left(p_{1}^{\prime}, p_{2}^{\prime}, \cdots, p_{J+I-1}^{\prime}\right)=\left(p_{1}, p_{2}, \cdots, \hat{p}_{i}, \cdots, p_{J+I}\right), \\
& Q^{\prime}=\left(q_{1}^{\prime}, q_{2}^{\prime}, \cdots, q_{J+I-1}^{\prime}\right)=\left(q_{1}, q_{2}, \cdots, \hat{q}_{i}, \cdots, q_{J+I}\right) ;
\end{aligned}
$$




$$
\begin{aligned}
& \left\{\begin{array}{l}
U^{0}=\left(u_{1}^{0}, u_{2}^{0}, \cdots, u_{J+I-1}^{0}\right)=\left(u_{1}, u_{2}, \cdots, \hat{u}_{i}, \cdots, u_{J+I}\right), \\
V^{0}=\left(v_{1}^{0}, v_{2}^{0}, \cdots, v_{J+I-1}^{0}\right)=\left(v_{1}, v_{2}, \cdots, \hat{v}_{i}, \cdots, v_{J+I}\right), \\
w^{0}=w, \quad x^{0}=x ;
\end{array}\right. \\
& \mathfrak{U}^{\prime}=\left(A, B^{\prime}, C^{\prime}, P^{\prime}, Q^{\prime}, r, s\right), \quad \mathfrak{H}^{0}=\left(U^{0}, V^{0}, w^{0}, x^{0}\right) .
\end{aligned}
$$

Then $\operatorname{sgn}(\mathfrak{U})=\operatorname{sgn}\left(\mathfrak{U}^{\prime}\right)$. Therefore putting $\delta^{\prime}=\left(\delta_{1}^{\prime}, \delta_{2}^{\prime}, \cdots, \delta_{n}^{\prime}\right)$ as

$$
\begin{cases}\left(\delta_{k+2 l+1}^{\prime}, \delta_{k+2 l+2}^{\prime}\right)=\left(e^{z_{l+1}}, e^{z_{l+1}}\right), \\ \delta_{k+2 l+\alpha}^{\prime}=\delta_{k+2 l+\alpha+2} & \text { for } p \leqslant \alpha \leqslant m-2, \\ \delta_{\alpha}^{\prime}=\delta_{\alpha} & \text { otherwise, }\end{cases}
$$

we get

$$
\begin{aligned}
\eta= & \sum_{\mathfrak{U}^{\prime}} \operatorname{sgn}\left(\mathfrak{U}^{\prime}\right) \xi_{k}\left(\delta_{1}^{\prime}, \delta_{2}^{\prime}, \cdots, \delta_{k}^{\prime} ; \lambda_{a_{1}}, \lambda_{a_{2}}, \cdots, \lambda_{a_{k}}\right) \\
& \times \prod_{1 \leqslant i \leqslant l+1} \eta_{2}\left(\delta_{k+2 \alpha-1}^{\prime}, \delta_{k+2 \alpha}^{\prime} ; \lambda_{b_{\alpha}^{\prime}}, \lambda_{c_{\alpha}^{\prime}}\right) \\
& \times \sum_{\mathfrak{u}^{\prime} 0} \prod_{1 \leqslant \alpha \leqslant J+I-1} \zeta_{2}\left(\delta_{u_{\alpha}^{\prime}}^{\prime}, \delta_{v_{\alpha}^{0}} ; \lambda_{p_{\alpha}^{\prime}}, \lambda_{q_{\alpha}^{\prime}}\right) \cdot \chi\left(\delta_{w^{0}}, \delta_{x^{0}} ; \lambda_{r}, \lambda_{s}\right) .
\end{aligned}
$$

Note that $\mathfrak{H}^{0}$ is a partition of $\Omega^{0}=\{k+2 l+1, k+2 l+2, \cdots, n\}-\{\bar{p}, \bar{p}+1\}$, which satisfies the analogous conditions as (7.11)-(7.13). Moreover $\Omega^{0}$ corresponds one to one onto $\Omega^{\prime}=\{k+2(l+1)+1, k+2(l+1)+2, \cdots, n\}$ in such a way that $\alpha \rightarrow \alpha+2$ if $\alpha<\bar{p}$ and $\alpha \rightarrow \alpha$ otherwise. Hence such $\mathfrak{H}^{0}$ corresponds uniquely to a partition $\mathfrak{H}^{\prime}=\left(U^{\prime}, V^{\prime}, w^{\prime}, x^{\prime}\right)$ of $\Omega^{\prime}$ satisfying the conditions corresponding to (7.11)-(7.13) (here $(k, l)$ is replaced by $(k, l+1)$ ), and so appearing in the expression of $\tilde{\kappa}_{j-2}^{k, l+1}$. Thus we see from (8.32) that the following equality holds :

$$
\tilde{\kappa}_{j-2}^{k, l+1}\left(h^{\prime}\right)=\eta,
$$

where $h^{\prime}$ denotes the element of $H_{j-2}^{k, l+1}$ with the co-ordinates $\delta^{\prime}$ in (8.31). This proves that the equations (4.35), (4.36) are satisfied and so the condition (III) in Case $\left(3^{+}\right)$.

8.8. The condition (III), Case (3-). The equations (4.38), (4.39) corresponding to $H_{j}^{k, l} \stackrel{\alpha, g}{\longrightarrow} H_{j}^{k, l+1}$ can be studied quite analogously as Case $\left(3^{+}\right)$, and we see that the condition (III) is also satisfied in Case ( $3^{-}$). The details are omitted here. Thus we complete the proof of the condition (III) in $\S \S 8.4-8.8$.

Now we have proved that the conditions (T), (I), (II) and (III) in Theorem 4 hold for the system of functions $\left\{\tilde{\kappa}_{j}^{k, l}\right\}$ given by the formulas (7.14). Hence by Theorem 4, the proof of our main theorem, Theorem 5 , is now complete. 


\section{§9. Some simple cases}

9.1. As is explained in $\S 5.4$, an equivalent class of discrete series representations is determined by

$$
\Lambda=\left(\lambda_{1}, \lambda_{2}, \cdots, \lambda_{n}\right)=\left(\nu_{1} l_{1}, \nu_{2} l_{2}, \cdots, \nu_{n} l_{n}\right)
$$

which satisfies the condition (5.7). Put

$$
\boldsymbol{l}=\left(l_{1}, l_{2}, \cdots, l_{n}\right), \quad \boldsymbol{\nu}=\left(\nu_{1}, \nu_{2}, \cdots, \nu_{n}\right) .
$$

Then, for certain fixed $\nu$, the formulas (7.14) of the characters can be reduced to more simple forms, valid for all $l$. These are the cases where all $\nu_{i}$ or all except one have the same sign. This will be seen below. The first case corresponds to the classes of the holomorphic discrete series and of their contragradient ones. Moreover it seems likely that $\nu$ classifies the discrete series representations from some point of view, at least from the appearances of the explicit forms of their characters.

9.2. The case where $\nu=(\nu, \nu, \cdots, \nu), \nu= \pm 1$. Let us consider the case where $\nu_{1}=\nu_{2}=\cdots=\nu_{n}=\nu= \pm 1$. Then $\nu_{p} \nu_{q}=1$ for any pair $p, q$, and hence we get by (6.2), (6.3) the following: for $p<q\left(\nu_{p} \nu_{q}=1\right)$,

$$
\begin{aligned}
\eta_{2}\left(\delta_{1}, \delta_{2} ; \lambda_{p}, \lambda_{q}\right) & =\left|-\delta^{-l_{p}}-\delta^{-l_{q}}\right|_{\delta_{\delta=\delta_{1}, \delta_{2}}}, \\
\zeta_{2}\left(\delta_{1}, \delta_{2} ; \lambda_{p}, \lambda_{q}\right) & =\chi\left(\delta_{1}, \delta_{2} ; \lambda_{p}, \lambda_{q}\right) \\
& =1-\delta^{-l_{p}}-\delta^{-\left.l_{q}\right|_{\delta_{\delta} \delta_{1}, \delta_{2}} .}
\end{aligned}
$$

Inserting them into (7.14), we get after a certain simple calculation the following character formula valid for all $l$.

THEOREM 6. In the case where $\nu_{1}=\nu_{2}=\cdots=\nu_{n}=\nu= \pm 1$, the character $\pi_{\Lambda}$ of the discrete series representations corresponding to $A$ in (9.1) is given as follows: let $h \in F_{+} \subset H_{j}^{k, l}$ be the element with the co-ordinates $\left(\delta_{1}, \delta_{2}, \cdots\right.$, $\left.\delta_{n}\right)$ in (5.1), then

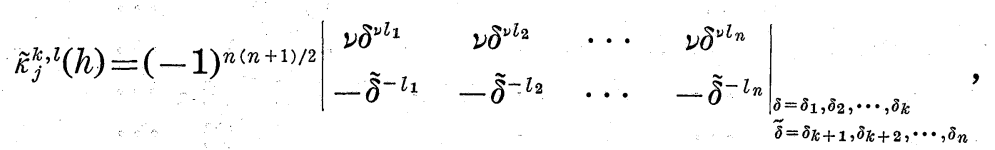

where the right hand side denotes the determinant of order $n$ whose $i$-th row is equal to $\left(\nu \delta_{i}^{\nu l_{1}}, \nu \delta_{i}^{\nu l_{2}}, \cdots, \nu \delta_{i}^{\nu l_{n}}\right)$ for $1 \leqslant i \leqslant k$, and to $\left(-\delta_{i}^{-l_{1}},-\delta_{i}^{-l_{2}}, \cdots\right.$, $\left.-\delta_{i}^{-l_{n}}\right)$ for $k+1 \leqslant i \leqslant n$.

Proof. Put $D=(k+2 l+1, k+2 l+2, \cdots, n)$ and denote by $\operatorname{sgn}(D ; U \cdot V$, $w, x)$ the sign of the permutation $(U \cdot V, w, x)$ of $D$. Then we get 


$$
\sum_{\mathfrak{u}} \operatorname{sgn}(D ; U \cdot V, w, x)=1 \text {, }
$$

where the sum runs over all $\mathfrak{U}=(U, V, w, x)$ satisfying the conditions (7.11)(7.13). Using this identity, we get the above formula (9.5) from (7.14).

Q.E.D.

ANOTHER PROOF. It is sufficient by Theorem 4, to prove that the conditions (T), (I), (II) and (III) hold for the system of functions $\left\{\tilde{\kappa}_{j}^{k, l}\right\}$ given by (9.5). This can be done directly in this special case much easier than the general case.

Q.E.D.

Note that the Graev's method for $S U(p, q)$ in [1] can be applied to the holomorphic discrete series of $G_{n} \cong S p(n, \boldsymbol{R})$. By this method, we get the character formula of such representations, which is exactly equal to (9.5) for $\nu=1$. The formula (9.5) for $\nu=-1$ gives the character formula of the contragradient representations of the holomorphic discrete series.

9.3. The case where $\nu=(\nu, \nu, \cdots, \nu,-\nu, \nu, \cdots, \nu), \nu= \pm 1$. Let us consider the case where $\nu_{i}=\nu$ except $i=y$ and $\nu_{y}=-\nu$ for some $y(1 \leqslant y \leqslant n)$. In this case we can also get from (7.14) a rather simple formula of $\tilde{\kappa}_{j}^{k, l}$ for every $(k, l, j)$. But the situation is considerably complicated in comprison with the case treated above. Put

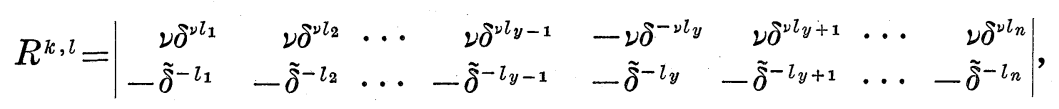

where $\delta$ runs over $\delta_{1}, \delta_{2}, \cdots, \delta_{k}$, and $\tilde{\delta}$ runs over $\delta_{k+1}, \delta_{k+2}, \cdots, \delta_{n}$ in this order

$$
\begin{aligned}
& S^{k, l}=\sum_{i=1}^{l}\left|\begin{array}{cccccccc}
\nu \delta^{\nu l_{1}} & \nu \delta^{\nu l_{2}} & \cdots & \nu \delta^{\nu l_{y-1}} & 0 & \nu \delta^{\nu l_{y+1}} & \cdots & \nu \delta^{\nu \nu l_{n}} \\
-\tilde{\delta}^{-l_{1}} & -\tilde{\delta}^{-l_{2}} & \cdots & -\tilde{\delta}^{-l_{y-1}} & 0 & -\tilde{\delta}^{-l_{y+1}} & \cdots & -\tilde{\delta}^{-t_{n}} \\
-\delta_{k+12 i-1}^{-l_{1}} & -\delta_{k+2 i-1}^{-l_{2}} & \cdots & -\delta_{k+2 i-1}^{-l_{y}-1} & \delta_{k+2 i-1}^{-l_{y}}+\delta_{k+2 i-1}^{l_{y}} & 0 & \cdots & 0 \\
-\delta_{k+2 i}^{-l_{1}} & -\delta_{k+2 i}^{-l_{2}} & \cdots & -\delta_{k+2 i}^{-l_{y}-1} & \delta_{k+2 i}^{-l y}+\delta_{k+2 i}^{l_{y}} & 0 & \cdots & 0
\end{array}\right|
\end{aligned}
$$

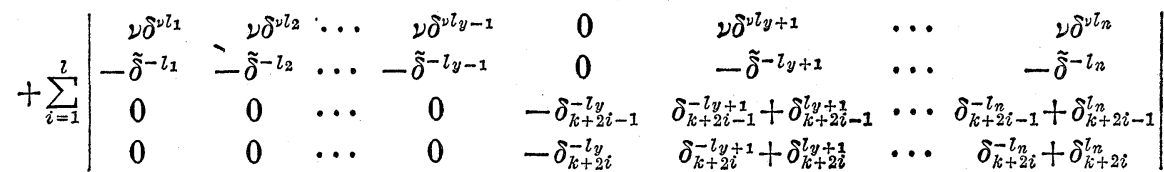

where, in two summands corresponding to $i, \delta$ runs over $\delta_{1}, \delta_{2}, \cdots, \delta_{k}$, and $\tilde{\delta}$ runs over $\delta_{k+1}, \delta_{k+2}, \cdots, \hat{\delta}_{k+2 i-1}, \hat{\delta}_{k+2 i}, \delta_{k+2 i+1}, \cdots, \delta_{n}$ in this order. Moreover put $m=n-k-2 l, \bar{p}=k+2 l+p, \bar{q}=k+2 l+q$, and 


$$
\begin{aligned}
& T_{j}^{k, l}=\sum_{\substack{1 \leqslant p<q \leqslant j \\
j+1 \leqslant p<q \leqslant m}}\left|\begin{array}{cccccccc}
\nu \delta^{\nu l_{1}} & \nu \delta^{\nu l_{2}} & \ldots & \nu \delta^{\delta l_{y-1}} & 0 & \nu \delta^{\nu l_{y+1}} & \ldots & \nu \delta_{n}^{\nu l_{n}} \\
-\tilde{\delta}^{-l_{1}} & -\tilde{\delta}^{-l_{2}} & \ldots & -\tilde{\delta}^{-l_{y-1}} & 0 & -\tilde{\delta}^{-l_{y+1}} & \ldots & -\tilde{\delta}^{-l_{n}} \\
-\delta_{\bar{p}}^{-l_{1}} & -\delta_{\bar{p}}^{-l_{2}} & \ldots & -\delta_{\bar{p}}^{-l_{y-1}} & 0 & 0 & \ldots & 0 \\
0 & 0 & \ldots & 0 & \delta_{\bar{q}}^{-l_{y}}+\delta_{\bar{q}}^{l_{y}} & 0 & \ldots & 0
\end{array}\right| \\
& +\sum_{\substack{1 \leqslant p<r \leqslant j \\
j+1 \leqslant p<q \leqslant m}}\left|\begin{array}{cccccccc}
\nu \delta^{\nu l_{1}} & \nu \delta^{\nu l_{2}} & \cdots & \nu \delta^{\nu l_{y-1}} & 0 & \nu \delta^{\nu l_{y+1}} & \cdots & \nu \delta^{\nu i_{n}} \\
-\tilde{\delta}^{-l_{1}} & -\tilde{\delta}^{-l_{2}} & \cdots & -\tilde{\delta}^{-l_{y-1}} & 0 & -\hat{\delta}^{-l_{y+1}} & \cdots & -\hat{\delta}^{-l_{n}} \\
0 & 0 & \cdots & 0 & -\delta_{\bar{p}}^{-l_{y}} & 0 & \cdots & 0 \\
0 & 0 & \cdots & 0 & 0 & \delta_{\bar{q}}^{-l_{y+1}}+\delta_{\bar{q}}^{l_{y+1}} & \cdots & \delta_{\bar{q}}^{-l_{n}}+\delta_{\bar{q}}^{l_{n}}
\end{array}\right| \text {, }
\end{aligned}
$$

where, in two summands corresponding to $(p, q)$, $\delta$ runs over $\delta_{1}, \delta_{2}, \cdots, \delta_{k}$, and $\tilde{\delta}$ runs over $\delta_{k+1}, \delta_{k+2}, \cdots, \hat{\delta}_{\bar{p}}, \cdots, \hat{\delta}_{\bar{q}}, \cdots, \delta_{n}$ in this order.

Then we get the following theorem.

THEOREM 7. Assume that $\nu$ satisfies that $\nu_{i}=\nu$ for $i \neq y$ and $\nu_{y}=-\nu$, for some $y$. Then the character $\pi_{A}$ of the discrete series representations corresponding to $\Lambda$ in (9.1) is given as follows: let $h \in F_{+} \subset H_{j}^{k, l}$ be the element with the co-ordinates $\left(\delta_{1}, \delta_{2}, \cdots, \delta_{n}\right)$ in $(5.1)$, then

$$
\tilde{\kappa}_{j}^{k, l}(h)=(-1)^{n(n+1) / 2}\left\{R^{k, l}+S^{k, l}+2 T_{j}^{k, l}\right\},
$$

where $R^{k, l}, S^{k, l}$ and $T_{j}^{k, l}$ are given above.

Note that when $y=n$, the second terms in $S^{k, l}$ and $T_{j}^{k, l}$ disappear, and the latter can be expressed by a single determinant of order $n$.

The proof of this theorem can be given quite analogously as for Theorem 6. If we start from the formula (7.14), the essential part of the calculation is to deduce from (7.14) the expression of $\tilde{\kappa}_{n}^{0,0}$ by means of $R^{0,0}$ and $T_{n}^{0,0}$ (for $\tilde{\kappa}_{n}^{0,0}, S^{0,0}=0$ ). The details of the proof are omitted here because they are not difficult but complicated.

As is suggested by this theorem, in the rested cases of $\nu$, the formula (7.14) of $\tilde{\kappa}_{j}^{k, l}$ can not be reduced to more simple form.

Note. When $\nu_{2 i-1}=1$ and $\nu_{2 i}=-1$ for any $i$, there appears the number $2^{n}$ or $-2^{n}$ in the coefficients of $\exp \{w \Lambda(\log h)\}$ in $\tilde{\kappa}_{n}^{0,0}$ for some $w \in W\left(\mathfrak{G}_{c}^{0,0}\right)$. We wonder whether all coefficients in $\tilde{k}_{j}^{k, l}$ are bounded by $2^{n}$ or not in absolute values.

\section{References}

[1] M. I. Graev: Unitary representations of real simple Lie groups (in Russian), Trudy Moskov. Mat. Obšč., 7 (1958), 335-389.

[2] Harish-Chandra:

(a) Some results on an invariant integral on a semi-simple Lie algebra, Ann. of Math., 80 (1964), 551-593.

(b) Invariant eigendistributions on a semisimple Lie group, Trans. Amer. 
Math. Soc., 119 (1965), 457-508.

(c) Discrete series for semisimple Lie groups, I, II, Acta Math., 113 (1965), 241-318; 116 (1966), 1-111.

[ 3] S. Helgason: Differential geometry and symmetric spaces, Academic Press, 1962.

[4] T. Hirai:

(a) Invariant eigendistributions of Laplace operators on real simple Lie groups, I. Case of $S U(p, q)$, Japan. J. Math., 39 (1970), 1-68.

(b) Explicit forms of the characters of discrete series representations of semisimple Lie groups, Proc. of Symp. in Pure Math., vol. 26, 281-287, Amer. Math. Soc., 1973.

(c) Invariant eigendistributions of Laplace operators on real simple Lie groups, II. General theory for semisimple Lie groups, Japan. J. Math., New Ser. 2 (1976), 27-89.

(d) - III. Methods of construction for semisimple Lie groups, ibid., 2 (1976), 269-341.

(e) Explicit forms of the characters of discrete series representations for $\operatorname{Spin}(p, q)$, preprint.

[5] W. Schmid:

(a) On the characters of the discrete series (the hermitian case), Invent. Math. 30 (1975), 47-144.

(b) Some remarks about the discrete series characters of $S p(n, \boldsymbol{R})$, Proc. of the Colloquium on Noncommutative Harmonic Analysis, Marseille, 1974. Lecture Notes in Mathematics no.466, Springer.

[6] M. Sugiura: Conjugate classes of Cartan subalgebras in real semisimple Lie algebras, J. Math. Soc. Japan, 11 (1959), 374-434.

Department of Mathematics

KYOTO UNIVERSITY

Куото 606

JAPAN 